\title{
The light and heavy Higgs interpretation of the MSSM
}

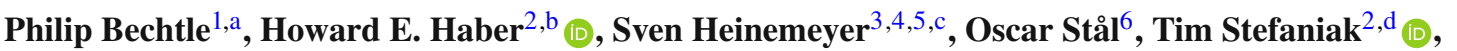 \\ Georg Weiglein $^{7, e}$, Lisa Zeune $^{8, f}$ \\ ${ }^{1}$ Physikalisches Institut der Universität Bonn, Nußallee 12, 53115 Bonn, Germany \\ ${ }^{2}$ Santa Cruz Institute for Particle Physics (SCIPP) and Department of Physics, University of California, Santa Cruz, 1156 High Street, Santa Cruz, \\ CA 95060, USA \\ ${ }^{3}$ Campus of International Excellence UAM+CSIC, Cantoblanco, 28049 Madrid, Spain \\ ${ }^{4}$ Instituto de Física Teórica, (UAM/CSIC), Universidad Autónoma de Madrid, Cantoblanco, 28049 Madrid, Spain \\ ${ }^{5}$ Instituto de Física de Cantabria (CSIC-UC), 39005 Santander, Spain \\ ${ }^{6}$ Department of Physics, The Oskar Klein Centre, Stockholm University, 10691 Stockholm, Sweden \\ ${ }^{7}$ Deutsches Elektronen-Synchrotron DESY, Notkestraße 85, 22607 Hamburg, Germany \\ ${ }^{8}$ Nikhef, Theory Group, Science Park 105, 1098 XG Amsterdam, The Netherlands
}

Received: 10 August 2016 / Accepted: 20 December 2016 / Published online: 3 February 2017

(c) The Author(s) 2017. This article is published with open access at Springerlink.com

\begin{abstract}
We perform a parameter scan of the phenomenological Minimal Supersymmetric Standard Model (pMSSM) with eight parameters taking into account the experimental Higgs boson results from Run I of the LHC and further lowenergy observables. We investigate various MSSM interpretations of the Higgs signal at $125 \mathrm{GeV}$. First, we consider the case where the light $\mathcal{C} \mathcal{P}$-even Higgs boson of the MSSM is identified with the discovered Higgs boson. In this case it can impersonate the SM Higgs-like signal either in the decoupling limit, or in the limit of alignment without decoupling. In the latter case, the other states in the Higgs sector can also be light, offering good prospects for upcoming LHC searches and for searches at future colliders. Second, we demonstrate that the heavy $\mathcal{C P}$-even Higgs boson is still a viable candidate to explain the Higgs signal - albeit only in a highly constrained parameter region, that will be probed by LHC searches for the $\mathcal{C} \mathcal{P}$-odd Higgs boson and the charged Higgs boson in the near future. As a guidance for such searches we provide new benchmark scenarios that can be employed to maximize the sensitivity of the experimental analysis to this interpretation.
\end{abstract}

\footnotetext{
a e-mail: bechtle@physik.uni-bonn.de

be-mail: haber@scipp.ucsc.edu

c e-mail: Sven.Heinemeyer@cern.ch

de-mail: tistefan@ucsc.edu

e e-mail: Georg.Weiglein@desy.de

fe-mail: lisa.zeune@nikhef.nl
}

\section{Introduction}

The discovery of a Higgs-like scalar boson in Run I of the large hadron collider (LHC) [1,2] marks a milestone in the exploration of electroweak symmetry breaking (EWSB). Within experimental and theoretical uncertainties, the properties of the new particle are compatible with the Higgs boson of the Standard Model (SM) [3]. However, a variety of other interpretations of the Higgs signal are possible, corresponding to very different underlying physics. Here, a prime candidate for the observed scalar boson is a $\mathcal{C P}$ even Higgs boson of the Minimal Supersymmetric Standard Model (MSSM) [4-6], as it possesses SM Higgs-like properties over a significant part of the model parameter space with only small deviations from the SM in the Higgs production and decay rates [7].

One of the main tasks of the LHC Run II will be to determine whether the observed scalar boson forms part of the Higgs sector of an extended model. In contrast to the SM, two Higgs doublets are needed in the MSSM to give mass to upand down-type fermions. The extended Higgs sector entails the existence of five scalar bosons, namely a light and heavy $\mathcal{C P}$-even Higgs bosons, $h$ and $H$, a $\mathcal{C P}$-odd Higgs boson, $A$, and a pair of charged Higgs bosons, $H^{ \pm}$. Mixing between the neutral $\mathcal{C P}$-even and $\mathcal{C} \mathcal{P}$-odd states are possible in the $\mathcal{C P}$ violating case [8-13], which we will not considered here. At lowest order, the Higgs sector of the MSSM can be fully specified in terms of the $W$ and $Z$ boson masses, $M_{W}$ and $M_{Z}$, the $\mathcal{C P}$-odd Higgs boson mass, $M_{A}$, and $\tan \beta \equiv v_{U} / v_{D}$, the ratio of the two neutral Higgs vacuum expectation values. However, higher-order corrections are crucial for a pre- 
cise prediction of the MSSM Higgs boson properties and introduce dependences on other model parameters, see e.g. Refs. [14-16] for reviews.

Many fits for the Higgs rates in various models and within the effective field theory approach have been performed over the last years, see e.g. Refs. [17, 18]. Focusing on the MSSM, recent fits have shown that the interpretation of the observed scalar as the light $\mathcal{C} \mathcal{P}$-even MSSM Higgs boson ("light Higgs case") is a viable possibility, providing a very good description of all data [19-24]; see also Refs. [25-27] for global fits including also astrophysical data. In the light-Higgs case, decoupling of the heavy Higgs bosons $\left(M_{A} \gg M_{Z}\right)$ [28-31] naturally explains the SM-like couplings of the light MSSM Higgs boson $h$ [7]. Another interesting possibility to explain the SM-like behavior of $h$ without decoupling in the MSSM - the so-called limit of alignment without decoupling - has been outlined in Refs. [32,33], relying on an (accidental) cancellation of tree-level and loop contributions in the $\mathcal{C P}$-even Higgs boson mass matrix. This led to the definition of a specific benchmark scenario [33], which has since been ruled out in the interesting low $M_{A}$ region via $p p \rightarrow H / A \rightarrow \tau^{+} \tau^{-}$ searches [23].

Alternatively, it was demonstrated that the heavy $\mathcal{C P}$-even Higgs boson can also be identified with the observed signal [7, 19,34-37] ("heavy Higgs case"). ${ }^{1}$ In this scenario all five MSSM Higgs bosons are relatively light, and in particular the lightest $\mathcal{C P}$-even Higgs boson has a mass (substantially) smaller than $125 \mathrm{GeV}$ with suppressed couplings to gauge bosons. This led to the development of the low$M_{H}$ benchmark scenario [43]. This particular scenario has meanwhile been ruled out by ATLAS and CMS searches for a light charged Higgs boson [44,45]. However the heavy Higgs interpretation in the MSSM remains viable, as we will discuss in this paper.

The questions arise, whether, and if so by how much, the MSSM can improve the theoretical description of the experimental data compared to the SM, and which parts of the MSSM parameter space are favored. In a previous analysis [19] we analyzed these questions within the MSSM. We performed a scan over the seven most relevant parameters for MSSM Higgs boson phenomenology, taking into account the data up to July 2012, which showed in particular some enhancement in the measured rate for $h \rightarrow \gamma \gamma$. We found that both the light and the heavy Higgs case provided a good fit to the data. In particular, the MSSM light Higgs case gave a better fit than the SM when the data in the $\gamma \gamma$ channel and low-energy data was included.

\footnotetext{
${ }^{1}$ Such a situation is more common in extensions of the MSSM. In particular, in the NMSSM it occurs generically if the singlet-like $\mathcal{C} \mathcal{P}$-even state is lighter than the doublet-like Higgs bosons, see e.g. Refs. [3842].
}

The situation has changed in several respects with the release of additional Higgs data by the ATLAS and CMS Collaborations [46]. In particular, the final data obtained in the LHC Run I does not show a significant enhancement over the SM prediction in the $\gamma \gamma$ channel anymore, and the heavy Higgs case is much more restricted due to light charged Higgs boson searches. The main aim of the present paper is to study the MSSM Higgs sector in full detail taking into account the current experimental data and in particular the final LHC Run I results, and to propose paths towards a complete exploration of the heavy Higgs case at the LHC in the ongoing Run II. We incorporate the available measurements of the Higgs boson mass and signal strengths, as well as measurements of the relevant low-energy observables. Furthermore we take into account all relevant constraints from direct Higgs and supersymmetric (SUSY) particle searches. We investigate whether the MSSM can still provide a good theoretical description of the current experimental data, and which parts of the parameter space of the MSSM are favored. Within the light Higgs case we analyze the situation with very large $M_{A}$ (decoupling), as well as for small/moderate $M_{A}$ (alignment without decoupling). We also investigate the feasibility of the heavy Higgs case and define new benchmark scenarios in which this possibility is realized, in agreement with all current Higgs constraints.

The paper is organized as follows. We employ the phenomenological MSSM with 8 parameters ( $p M S S M 8$ ), which is introduced in detail in Sect. 2. In this section, we also expand upon the theoretical background of the two possible limits that lead to alignment in the $\mathcal{C P}$ even Higgs sector, i.e. when one of the $\mathcal{C} \mathcal{P}$-even neutral MSSM Higgs bosons behaves like the SM Higgs boson. In particular, we outline how leading two-loop effects on the conditions for alignment can be assessed and present a brief quantitative discussion of these effects. $^{2}$ The parameter scan with $\mathcal{O}\left(10^{7}\right)$ sampling points, the techniques to achieve good coverage, as well as the considered experimental observables and constraints are described in Sect. 3. In Sect. 4 we present our results for the best-fit points and the preferred parameter regions for the light Higgs and the heavy Higgs interpretation. The effects of the Higgs mass and Higgs rates measurements, precision observables, and direct Higgs and SUSY searches are discussed, and the phenomenology of the other MSSM Higgs states is outlined. In particular, in Sect. 4.4 we propose new benchmark scenarios for the study of the heavy Higgs case, which can be probed at the LHC Run II. We conclude in Sect. 5. In Appendix A, we discuss the extent of the tuning associated with the regions of the MSSM parameter space that exhibit approximate Higgs alignment without decoupling. Finally in Appendix B, we provide tables listing the

\footnotetext{
${ }^{2}$ More details will be presented in a separate publication [47].
} 
signal strength measurements from ATLAS, CMS and the Tevatron (DØ and CDF) that are included in our analysis.

\section{Theoretical background}

\subsection{The MSSM Higgs sector}

In this section we briefly review the most important features of the MSSM Higgs sector and motivate the choice of the eight free pMSSM parameters in our scan. We provide a detailed description of the relevant MSSM parameter sectors and our notations, which remain unchanged compared to [19].

In the supersymmetric extension of the SM, an even number of Higgs multiplets consisting of pairs of Higgs doublets with opposite hypercharge is required to avoid anomalies due to the supersymmetric Higgsino partners. Consequently the MSSM employs two Higgs doublets, denoted by $H_{D}$ and $H_{U}$, with hypercharges -1 and +1 , respectively. After minimizing the scalar potential, the neutral components of $H_{D}$ and $H_{U}$ acquire vacuum expectation values (vevs), $v_{D}$ and $v_{U}$. Without loss of generality, we assume that the vevs are real and non-negative (this can be achieved by appropriately rephasing the Higgs doublet fields). The vevs are normalized such that

$v^{2} \equiv v_{D}^{2}+v_{U}^{2} \simeq(246 \mathrm{GeV})^{2}$.

In addition, we define

$\tan \beta \equiv v_{U} / v_{D}$.

Without loss of generality, we may assume that $0 \leq \beta \leq \frac{1}{2} \pi$ (i.e., $\tan \beta$ is non-negative). This can always be achieved by a rephasing of one of the two Higgs doublet fields.

The two-doublet Higgs sector gives rise to five physical Higgs states. The mass eigenstates correspond to the neutral Higgs bosons $h, H$ (with $M_{h}<M_{H}$ ) and $A$, and the charged Higgs pair $H^{ \pm}$. Neglecting possible $\mathcal{C} \mathcal{P}$-violating contributions of the soft-supersymmetry-breaking terms (which can modify the neutral Higgs properties at the loop level), $h$ and $H$ are the light and heavy $\mathcal{C} \mathcal{P}$-even Higgs bosons, and $A$ is $\mathcal{C P}$-odd.

At lowest order, the MSSM Higgs sector is fully described by $M_{Z}$ and two MSSM parameters, often chosen as the $\mathcal{C} \mathcal{P}$ odd Higgs boson mass, $M_{A}$, and $\tan \beta$. In the MSSM at the tree-level the mass of the light $\mathcal{C P}$-even Higgs boson does not exceed $M_{Z}$. However, higher order corrections to the Higgs masses are known to be sizable and must be included, in order to be consistent with the observed Higgs signal at $125 \mathrm{GeV}$ [3]. Particularly important are the oneand two-loop contributions from top quarks and their scalar top ("stop") partners. In order to shift the mass of $h$ up to $125 \mathrm{GeV}$, large radiative corrections are necessary, which require a large splitting in the stop sector and/or heavy stops. For large values of $\tan \beta$, the sbottom contributions to the radiative corrections also become sizable. The stop (sbottom) sector is governed by the soft SUSY-breaking mass parameter $M_{\tilde{t}_{L}}$ and $M_{\tilde{t}_{R}}\left(M_{\tilde{b}_{L}}\right.$ and $\left.M_{\tilde{b}_{R}}\right)$, where SU(2) gauge invariance requires $M_{\tilde{t}_{L}}=M_{\tilde{b}_{L}}$, the trilinear coupling $A_{t}$ $\left(A_{b}\right)$ and the Higgsino mass parameter $\mu$.

To achieve a good sampling of the full MSSM parameter space with $\mathcal{O}\left(10^{7}\right)$ points, we restrict ourselves to the eight MSSM parameters

$\tan \beta, \quad M_{A}, \quad M_{\tilde{q}_{3}}, \quad A_{f}, \quad \mu, \quad M_{\tilde{\ell}_{3}}, \quad M_{\tilde{\ell}_{1,2}}, \quad M_{2}$

most relevant for phenomenology of the Higgs sector (the scan ranges will be given in Sect. 3.1), under the assumption that the third generation squark and slepton parameters are universal. That is, we take $M_{\tilde{q}_{3}}:=M_{\tilde{t}_{L}}\left(=M_{\tilde{b}_{L}}\right)=M_{\tilde{t}_{R}}=$ $M_{\tilde{b}_{R}}, M_{\tilde{\ell}_{3}}:=M_{\tilde{\tau}_{L}}=M_{\tilde{\tau}_{R}}=M_{\tilde{v}_{\tau}}$ and $A_{f}:=A_{t}=A_{b}=$ $A_{\tau}$. Note that the soft SUSY-breaking mass parameter in the stau sector, $M_{\tilde{\ell}_{3}}$, can significantly impact the Higgs decays as light staus can modify the loop-induced diphoton decay. $M_{\tilde{\ell}_{3}}$ is therefore taken as an independent parameter in our scans. Even though the other slepton and gaugino parameters are generally of less importance for the Higgs phenomenology, we scan over the $\mathrm{SU}(2)$ gaugino mass parameter $M_{2}$ as well as over the mass parameter of the first two generation sleptons, $M_{\tilde{\ell}_{1,2}}$, (assumed to be equal) as these parameters are important for the low-energy observables included in our analysis. The remaining MSSM parameters are fixed,

$$
\begin{array}{r}
M_{\tilde{q}_{L}}=M_{\tilde{q}_{R}}(q=c, s, u, d)=1500 \mathrm{GeV}, \\
M_{3}=m_{\tilde{g}}=1500 \mathrm{GeV} .
\end{array}
$$

We choose relatively high values for the squark and gluino mass parameters, which have a minor impact on the Higgs sector, in order to be in agreement with the limits from direct SUSY searches. Finally, the $\mathrm{U}(1)_{\mathrm{Y}}$ gaugino mass parameter, $M_{1}$, is fixed via the GUT relation

$M_{1}=\frac{5}{3} \frac{s_{W}^{2}}{c_{W}^{2}} \quad M_{2} \approx \frac{1}{2} M_{2}$,

with $s_{W}=\sqrt{1-c_{W}^{2}}$ and $c_{W}=M_{W} / M_{Z}$. For more details on the definition of the MSSM parameters, we refer to [19].

\subsection{The Higgs alignment limit}

In light of the Higgs data, which indicates that the properties of the observed Higgs boson are SM-like, we seek to explore the region of the MSSM parameter space that yields a SMlike Higgs boson. In general, a SM-like Higgs boson arises if one of the neutral Higgs mass eigenstates is approximately 
aligned with the direction of the Higgs vev in field space. Thus, the limit of a SM Higgs boson is called the alignment limit.

To analyze the alignment limit, it is convenient to define

$\left(\Phi_{1}\right)^{i}=\epsilon_{i j}\left(H_{D}^{*}\right)^{j}, \quad\left(\Phi_{2}\right)^{i}=\left(H_{U}\right)^{i}$,

where $\epsilon_{12}=-\epsilon_{21}=1$ and $\epsilon_{11}=\epsilon_{22}=0$, and there is an implicit sum over the repeated $\mathrm{SU}(2)$ index $j=1,2$. For consistency of the notation, we denote the corresponding neutral Higgs vevs by $v_{1} \equiv v_{D}$ and $v_{2} \equiv v_{U}$. We now define the following linear combinations of Higgs doublet fields,

$\mathcal{H}_{1}=\left(\begin{array}{c}H_{1}^{+} \\ H_{1}^{0}\end{array}\right) \equiv \frac{v_{1} \Phi_{1}+v_{2} \Phi_{2}}{v}$,

$\mathcal{H}_{2}=\left(\begin{array}{c}H_{2}^{+} \\ H_{2}^{0}\end{array}\right) \equiv \frac{-v_{2} \Phi_{1}+v_{1} \Phi_{2}}{v}$

such that $\left\langle H_{1}^{0}\right\rangle=v / \sqrt{2}$ and $\left\langle H_{2}^{0}\right\rangle=0$, which defines the socalled Higgs basis [48-50]. ${ }^{3}$ It is straightforward to express the scalar Higgs potential in terms of the Higgs basis fields $\mathcal{H}_{1}$ and $\mathcal{H}_{2}$,

$$
\begin{aligned}
\mathcal{V}= & \cdots+\frac{1}{2} Z_{1}\left(\mathcal{H}_{1}^{\dagger} \mathcal{H}_{1}\right)^{2}+\cdots \\
& +\left[\frac{1}{2} Z_{5}\left(\mathcal{H}_{1}^{\dagger} \mathcal{H}_{2}\right)^{2}+Z_{6}\left(\mathcal{H}_{1}^{\dagger} \mathcal{H}_{1}\right)\left(\mathcal{H}_{1}^{\dagger} \mathcal{H}_{2}\right)+\text { h.c. }\right]+\cdots
\end{aligned}
$$

where the most important terms of the scalar potential are highlighted above. The quartic couplings $Z_{1}, Z_{5}$ and $Z_{6}$ are linear combinations of the quartic couplings that appear in the MSSM Higgs potential expressed in terms of $H_{D}$ and $H_{U}$. In particular, at tree-level,

$$
\begin{aligned}
& Z_{1}=\frac{1}{4}\left(g^{2}+g^{\prime 2}\right) c_{2 \beta}^{2}, \\
& Z_{5}=\frac{1}{4}\left(g^{2}+g^{\prime 2}\right) s_{2 \beta}^{2}, \\
& Z_{6}=-\frac{1}{4}\left(g^{2}+g^{\prime 2}\right) s_{2 \beta} c_{2 \beta},
\end{aligned}
$$

where $g$ and $g^{\prime}$ are the $\mathrm{SU}(2)$ and $\mathrm{U}(1)_{\mathrm{Y}}$ gauge couplings, respectively, $c_{2 \beta} \equiv \cos 2 \beta$ and $s_{2 \beta} \equiv \sin 2 \beta$. Hence, the $Z_{i}$ are $\mathcal{O}(1)$ parameters.

One can then evaluate the squared-mass matrix of the neutral $\mathcal{C P}$-even Higgs bosons, with respect to the neutral Higgs states, $\left\{\sqrt{2} \operatorname{Re} H_{1}^{0}-v, \sqrt{2} \operatorname{Re} H_{2}^{0}\right\}$

$\mathcal{M}^{2}=\left(\begin{array}{cc}Z_{1} v^{2} & Z_{6} v^{2} \\ Z_{6} v^{2} & M_{A}^{2}+Z_{5} v^{2}\end{array}\right)$.

\footnotetext{
${ }^{3}$ Since the tree-level MSSM Higgs sector is $\mathcal{C} \mathcal{P}$-conserving, the Higgs basis is defined up to an overall sign ambiguity, where $\mathcal{H}_{2} \rightarrow-\mathcal{H}_{2}$. However, since we have adopted the convention in which $\tan \beta$ is nonnegative [cf. the comment below Eq. (2)], the overall sign of the Higgs basis field $\mathcal{H}_{2}$ is now fixed.
}

If $\sqrt{2} \operatorname{Re} H_{1}^{0}-v$ were a Higgs mass eigenstate, then its treelevel couplings to SM particles would be precisely those of the SM Higgs boson. This would correspond to the exact alignment limit. To achieve a SM-like neutral Higgs state, it is sufficient for one of the neutral Higgs mass eigenstates to be approximately given by $\sqrt{2} \operatorname{Re} H_{1}^{0}-v$. In light of the form of the squared-mass matrix given in (11), we see that a SM-like neutral Higgs boson can arise in two different ways:

1. $M_{A}^{2} \gg\left(Z_{1}-Z_{5}\right) v^{2}$. This is the so-called decoupling limit, where $h$ is SM-like and $M_{A} \sim M_{H} \sim M_{H^{ \pm}} \gg M_{h}$.

2. $\left|Z_{6}\right| \ll 1$. Then, $h$ is SM-like if $M_{A}^{2}+\left(Z_{5}-Z_{1}\right) v^{2}>0$ and $H$ is SM-like if $M_{A}^{2}+\left(Z_{5}-Z_{1}\right) v^{2}<0$.

In particular, the $\mathcal{C P}$-even mass eigenstates are:

$$
\left(\begin{array}{l}
H \\
h
\end{array}\right)=\left(\begin{array}{cc}
c_{\beta-\alpha} & -s_{\beta-\alpha} \\
s_{\beta-\alpha} & c_{\beta-\alpha}
\end{array}\right)\left(\begin{array}{c}
\sqrt{2} \operatorname{Re} H_{1}^{0}-v \\
\sqrt{2} \operatorname{Re} H_{2}^{0}
\end{array}\right),
$$

where $c_{\beta-\alpha} \equiv \cos (\beta-\alpha)$ and $s_{\beta-\alpha} \equiv \sin (\beta-\alpha)$ are defined in terms of the mixing angle $\alpha$ that diagonalizes the $\mathcal{C P}$-even Higgs squared-mass matrix when expressed in the original basis of scalar fields, $\left\{\sqrt{2} \operatorname{Re} \Phi_{1}^{0}-v_{1}, \sqrt{2} \operatorname{Re} \Phi_{2}^{0}-v_{2}\right\}$. Since the SM-like Higgs field is approximately $\sqrt{2} \operatorname{Re} H_{1}^{0}-v$, it follows that $h$ is SM-like if $\left|c_{\beta-\alpha}\right| \ll 1$ [51] and $H$ is SMlike if $\left|s_{\beta-\alpha}\right| \ll 1$ [52]. The case of a SM-like $H$ necessarily corresponds to alignment without decoupling.

In the case of exact alignment without decoupling, $Z_{6}=$ 0 , the tree-level couplings of the SM-like Higgs boson are precisely those of the Higgs boson of the Standard Model. Nevertheless, deviations from SM Higgs boson properties can arise due to two possible effects. First, there might exist new particles that enter in loops and modify the loop-induced Higgs couplings to $g g, \gamma \gamma$ and $Z \gamma$. For example, if $H$ is the SM-like Higgs boson, then the charged Higgs boson mass is not significantly larger than the observed Higgs mass, in which case the charged Higgs loop can shift the one-loop induced couplings of the observed Higgs boson to $\gamma \gamma$ and $Z \gamma$ [52]. Similarly, SUSY particles can give a contribution at the loop-level to other, at the tree-level SM-like, couplings. Second, there might exist new particles with mass less that half the Higgs mass, allowing for new decay modes of the SM-like Higgs boson. An example of this possibility arises if $H$ is the SM-like Higgs boson and $M_{h}<M_{H} / 2$, in which case the decay mode $H \rightarrow h h$ is allowed. Indeed, in the exact alignment limit where $s_{\beta-\alpha}=0$, the tree-level $H h h$ coupling in the MSSM is given by [53]

$g_{H h h}=\frac{g M_{Z}}{2 c_{W}}\left(1-3 \sin ^{2} 2 \beta\right)$.

The possibility of alignment without decoupling has been analyzed in detail in Refs. [31-33,51,52,54-56] (see also the " $\tau$-phobic" benchmark scenario in Ref. [57]). It was pointed out that exact alignment via $Z_{6}=0$ can only happen through 
an accidental cancellation of the tree-level terms with contributions arising at the one-loop level (or higher). In this case the Higgs alignment is independent of $M_{A}^{2}, Z_{1}$ and $Z_{5}$. This has two phenomenological consequences. First, the remaining Higgs states can be light, which would imply good prospects for LHC searches. Second, either the light or the heavy neutral Higgs mass eigenstate can be aligned with the SM Higgs vev and thus be interpreted as the SM-like Higgs boson observed at $125 \mathrm{GeV}$.

The leading one-loop contributions to $Z_{1}, Z_{5}$ and $Z_{6}$ proportional to $h_{t}^{2} m_{t}^{2}$, where

$h_{t}=\frac{\sqrt{2} m_{t}}{v s_{\beta}}$

is the top quark Yukawa coupling, have been obtained in Ref. [33] in the limit $M_{Z}, M_{A} \ll M_{S}$ (using results from Ref. [58]):

$$
\begin{aligned}
Z_{1} v^{2}= & M_{Z}^{2} c_{2 \beta}^{2} \\
& +\frac{3 m_{t}^{4}}{2 \pi^{2} v^{2}}\left[\ln \left(\frac{M_{S}^{2}}{m_{t}^{2}}\right)+\frac{X_{t}^{2}}{M_{S}^{2}}\left(1-\frac{X_{t}^{2}}{12 M_{S}^{2}}\right)\right], \\
Z_{5} v^{2}= & s_{2 \beta}^{2}\left\{M_{Z}^{2}+\frac{3 m_{t}^{4}}{8 \pi^{2} v^{2} s_{\beta}^{4}}\right. \\
& \left.\times\left[\ln \left(\frac{M_{S}^{2}}{m_{t}^{2}}\right)+\frac{X_{t} Y_{t}}{M_{S}^{2}}\left(1-\frac{X_{t} Y_{t}}{12 M_{S}^{2}}\right)\right]\right\}, \\
Z_{6} v^{2}= & -s_{2 \beta}\left\{M_{Z}^{2} c_{2 \beta}-\frac{3 m_{t}^{4}}{4 \pi^{2} v^{2} s_{\beta}^{2}}\right. \\
& \left.\times\left[\ln \left(\frac{M_{S}^{2}}{m_{t}^{2}}\right)+\frac{X_{t}\left(X_{t}+Y_{t}\right)}{2 M_{S}^{2}}-\frac{X_{t}^{3} Y_{t}}{12 M_{S}^{4}}\right]\right\},
\end{aligned}
$$

where $s_{\beta} \equiv \sin \beta, M_{S} \equiv \sqrt{m_{\tilde{t}_{1}} m_{\tilde{t}_{2}}}$ denotes the SUSY mass scale, given by the geometric mean of the light and heavy stop masses, and

$X_{t} \equiv A_{t}-\mu / \tan \beta, \quad Y_{t} \equiv A_{t}+\mu \tan \beta$.

In Eqs. (15)-(18), we have assumed for simplicity that $\mu$ and $A_{t}$ (as well as the gaugino mass parameters that contribute subdominantly at one-loop to the $Z_{i}$ ) are real parameters. That is, we are neglecting $\mathcal{C P}$-violating effects that can enter the MSSM Higgs sector via radiative corrections.

The approximate expression for $Z_{6} v^{2}$ given in (17) depends only on the unknown parameters $\mu, A_{t}, \tan \beta$ and $M_{S}$. Exact alignment arises when $Z_{6}=0$. Note that $Z_{6}=0$ trivially occurs when $\beta=0$ or $\frac{1}{2} \pi$ (corresponding to the vanishing of either $v_{1}$ or $v_{2}$ ). But, this choice of parameters is not relevant for phenomenology as it leads to a massless $b$ quark or $t$ quark, respectively, at tree-level. Henceforth, we assume that $\tan \beta$ is non-zero and finite. In our convention, $\tan \beta$ is positive with $0<\beta<\frac{1}{2} \pi$.

We can simplify the analysis of the condition $Z_{6}=0$ by solving Eq. (15) for $\ln \left(M_{S}^{2} / m_{t}^{2}\right)$ and inserting this result back into Eq. (17). The resulting expression for $Z_{6}$ now depends on $Z_{1}, \tan \beta$, and the ratios,

$\widehat{A_{t}} \equiv \frac{A_{t}}{M_{S}}, \quad \widehat{\mu} \equiv \frac{\mu}{M_{S}}$.

Using Eq. (18) to rewrite the final expression in terms of $\widehat{A}_{t}$ and $\widehat{\mu}$, we obtain,

$$
\begin{aligned}
Z_{6} v^{2}= & -\cot \beta\left\{M_{Z}^{2} c_{2 \beta}-Z_{1} v^{2}+\frac{3 m_{t}^{4} \widehat{\mu}\left(\widehat{A}_{t} \tan \beta-\widehat{\mu}\right)}{4 \pi^{2} v^{2} s_{\beta}^{2}}\right. \\
& \left.\times\left[\frac{1}{6}\left(\widehat{A}_{t}-\widehat{\mu} \cot \beta\right)^{2}-1\right]\right\} .
\end{aligned}
$$

Setting $Z_{6}=0$, we can identify $Z_{1} v^{2}$ with the mass of the observed (SM-like) Higgs boson (which may be either $h$ or $H$ depending on whether $s_{\beta-\alpha}$ is close to 1 or 0 , respectively). We can then numerically solve for $\tan \beta$ for given values of $\widehat{A}_{t}$ and $\widehat{\mu}$. The values of the real positive $\tan \beta$ solutions of $Z_{6}=0$ obtained by using the one-loop approximate formula given in Eq. (20) are illustrated by the contour plots shown in the three left panels of Fig. 1, where each panel corresponds to a different solution of $Z_{6}=0$. Note that at every point in the $\left(\widehat{\mu}, \widehat{A}_{t}\right)$ plane, the value of $M_{S}$ has been adjusted according to Eq. (15) such that the squared-mass of the SM-like Higgs boson in the alignment limit is given by $Z_{1} v^{2} \simeq(125 \mathrm{GeV})^{2}$. Taking the three left panels together, one can immediately discern the regions of zero, one, two and three positive $\tan \beta$ solutions of Eq. (20), and their corresponding values. A more detailed discussion of these solutions will be presented in a separate paper [47].

It is instructive to obtain an approximate analytic expression for the value of the largest real positive $\tan \beta$ solution. Assuming $\left|\widehat{\mu} \widehat{A}_{t}\right| \tan \beta \gg 1$ the following approximate alignment condition, first written in Ref. [33], is obtained,

$$
\begin{aligned}
\tan \beta & \simeq \frac{M_{h / H}^{2}+M_{Z}^{2}+\frac{3 m_{t}^{4} \widehat{\mu}^{2}}{8 \pi^{2} v^{2}}\left(\widehat{A}_{t}^{2}-2\right)}{\frac{m_{t}^{4} \widehat{\mu} \widehat{A}_{t}}{8 \pi^{2} v^{2}}\left(\widehat{A}_{t}^{2}-6\right)} \\
& \simeq \frac{127+3 \widehat{\mu}^{2}\left(\widehat{A}_{t}^{2}-2\right)}{\widehat{\mu} \widehat{A}_{t}\left(\widehat{A}_{t}^{2}-6\right)}
\end{aligned}
$$

where $M_{h / H}^{2} \simeq Z_{1} v^{2}$ denotes the (one-loop) mass of the SM-like Higgs boson obtained from Eq. (15), which could be either the light or heavy $\mathcal{C P}$-even Higgs boson. It is clear from Eq. (21) that a positive $\tan \beta$ solution exists if either $\widehat{\mu} \widehat{A}_{t}\left(\widehat{A}_{t}^{2}-6\right)>0$ and $\widehat{A}_{t}^{2}>2$, or if $\widehat{\mu} \widehat{A}_{t}\left(\widehat{A}_{t}^{2}-6\right)<0$, $\widehat{A}_{t}^{2}<2$ and $|\widehat{\mu}|$ is sufficiently large such that the numerator of Eq. (21) is negative. Keeping in mind that Eq. (21) was 
$\tan \beta \leq 5 \square \tan \beta \leq 10 \square \tan \beta \leq 15 \square \tan \beta \leq 25 \square \tan \beta \leq 50$

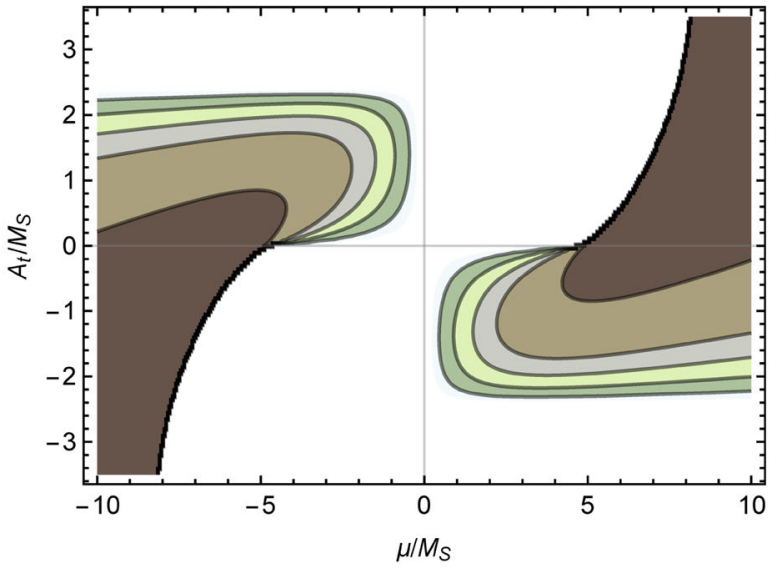

$\tan \beta \leq 5 \square \tan \beta \leq 10 \square \tan \beta \leq 15 \square \tan \beta \leq 25 \square \tan \beta \leq 50$

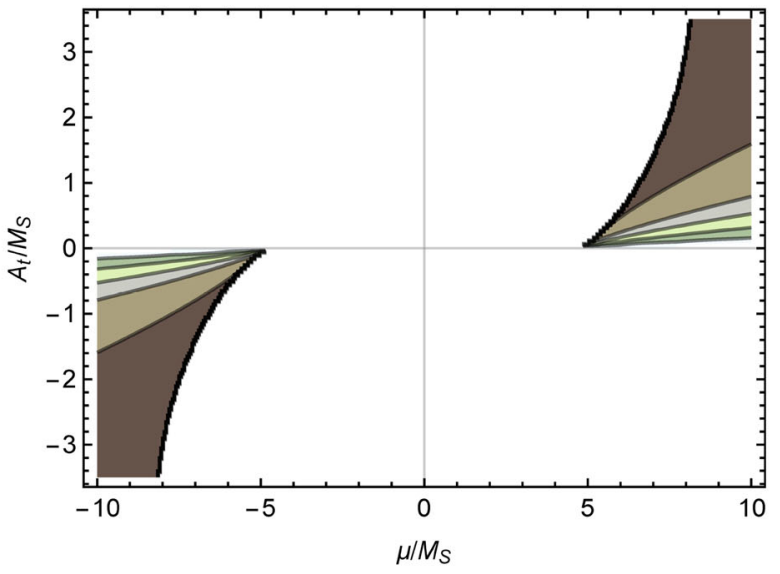

$\tan \beta \leq 5 \square \tan \beta \leq 10 \square \tan \beta \leq 15 \square \tan \beta \leq 25 \square \tan \beta \leq 50$

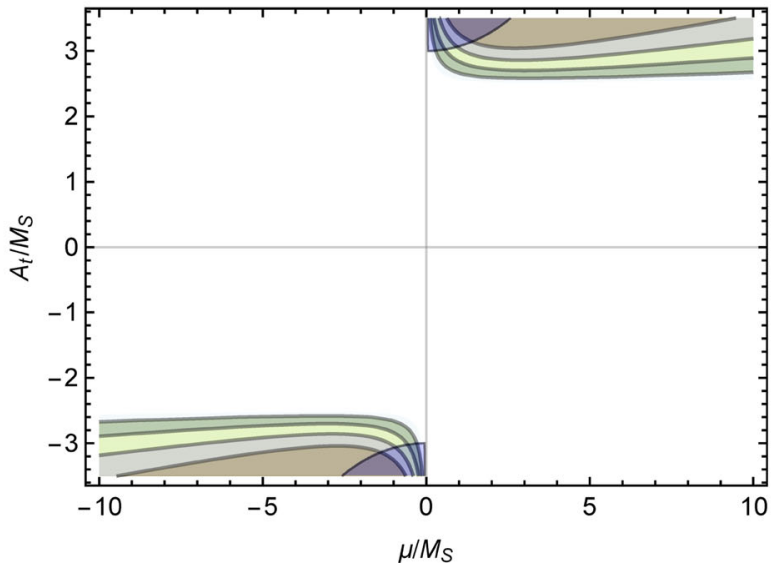

Fig. 1 Contours of $\tan \beta$ corresponding to exact alignment, $Z_{6}=0$, in the $\left(\mu / M_{S}, A_{t} / M_{S}\right)$ plane. $Z_{1}$ is adjusted to give the correct Higgs mass. The three left panels exhibit the approximate one-loop results; the three right panels exhibit the corresponding two-loop improved results. $\square \tan \beta \leq 5 \square \tan \beta \leq 10 \square \tan \beta \leq 15 \square \tan \beta \leq 25 \square \tan \beta \leq 50$

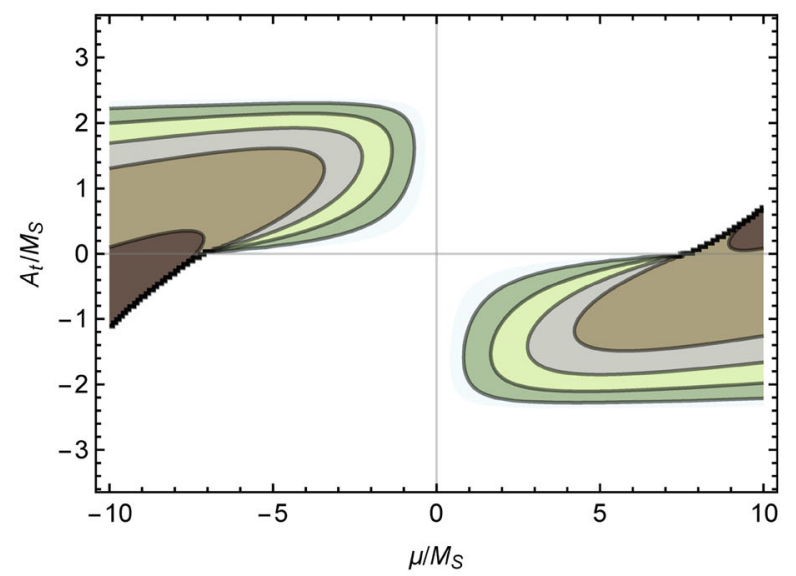

$\tan \beta \leq 5 \square \tan \beta \leq 10 \square \tan \beta \leq 15 \square \tan \beta \leq 25 \square \tan \beta \leq 50$

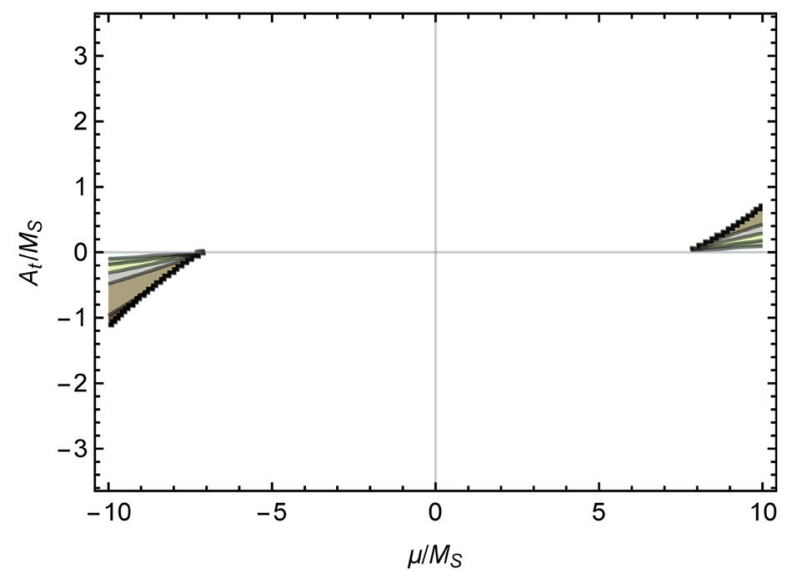

$\tan \beta \leq 5 \square \tan \beta \leq 10 \square \tan \beta \leq 15 \square \tan \beta \leq 25 \square \tan \beta \leq 50$

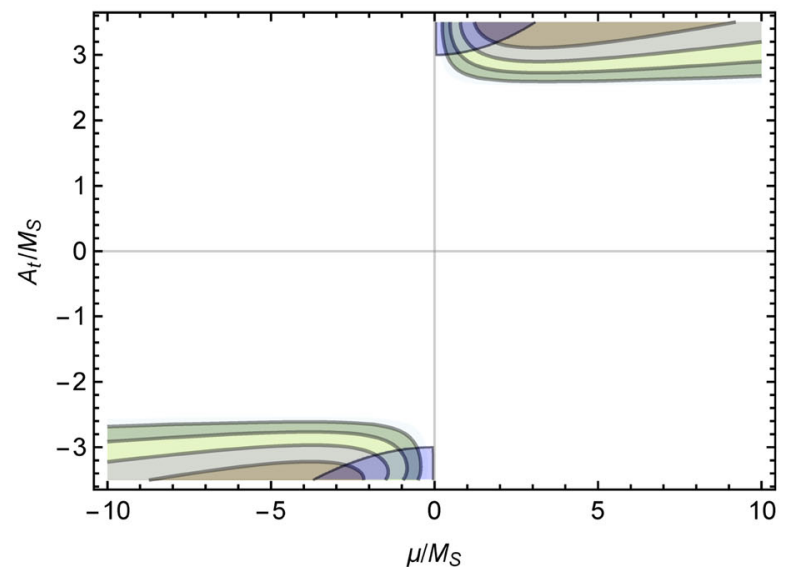

Taking the three panels on each side together, one can immediately discern the regions of zero, one, two and three values of $\tan \beta$ in which exact alignment is realized. In the overlaid blue regions we have (unstable) values of $\left|X_{t} / M_{S}\right| \geq 3$ 
derived under the assumption that $\widehat{\mu} \widehat{A}_{t} \tan \beta \gg 1$, one easily verifies that the largest of the three roots of Eq. (20) shown in Fig. 1 always satisfies the stated conditions above. Another consequence of Eq. (21) is that by increasing the value of $\left|\widehat{\mu} \widehat{A}_{t}\right|$ (in the region where $2<\widehat{A}_{t}^{2}<6$ ), it is possible to lower the $\tan \beta$ value at which alignment occurs.

If $\left|\widehat{A}_{t}\right| \ll 1$, then Eq. (21) is no longer a good approximation. Returning to Eq. (20), we set $\widehat{A}_{t}=0$ and again assume that $\tan \beta \gg 1$. We can then solve approximately for $\tan \beta$,

$$
\tan ^{2} \beta \simeq \frac{M_{Z}^{2}-M_{h / H}^{2}+\frac{3 m_{t}^{4} \widehat{\mu}^{2}}{4 \pi^{2} v^{2}}\left(\frac{1}{6} \widehat{\mu}^{2}-2\right)}{M_{Z}^{2}+M_{h / H}^{2}+\frac{3 m_{t}^{4} \widehat{\mu}^{2}}{4 \pi^{2} v^{2}}} .
$$

For example, in the parameter regime where $\widehat{A}_{t} \simeq 0$ and $|\widehat{\mu}| \gg 1$, we obtain $\tan \beta \simeq|\widehat{\mu}| / \sqrt{6}$.

The question of whether the light or the heavy $\mathcal{C P}$-even Higgs boson possesses SM-like Higgs couplings in the alignment without decoupling regime depends on the relative size of $Z_{1} v^{2}$ and $Z_{5} v^{2}+M_{A}^{2}$. Combining Eqs. (16) and (17), it follows that in the limit of exact alignment where $Z_{6}=0$, we identify $Z_{1} v^{2}$ as the squared mass of the observed SM-like Higgs boson and

$$
\begin{aligned}
Z_{5} v^{2}= & M_{Z}^{2}\left(1+c_{2 \beta}\right)+\frac{3 m_{t}^{4} \widehat{\mu}\left(\widehat{A}_{t}-\widehat{\mu} \cot \beta\right)}{8 \pi^{2} v^{2} s_{\beta}^{4}} \\
& \times\left\{s_{2 \beta}-\frac{1}{6}\left[\left(\widehat{A}_{t}^{2}-\widehat{\mu}^{2}\right) s_{2 \beta}-2 \widehat{A}_{t} \widehat{\mu} c_{2 \beta}\right]\right\} .
\end{aligned}
$$

We define a critical value of $M_{A}^{2}$,

$M_{A, c}^{2} \equiv \max \left\{\left(Z_{1}-Z_{5}\right) v^{2}, 0\right\}$,

where $Z_{1} v^{2}=(125 \mathrm{GeV})^{2}$ and $Z_{5} v^{2}$ is given by Eq. (23). Note further that the squared-mass of the non-SM-like $\mathcal{C P}$ even Higgs boson in the exact alignment limit, $M_{A}^{2}+Z_{5} v^{2}$, is positive, which implies that the minimum value possible for the squared-mass of the $\mathcal{C P}$-odd Higgs boson is

$M_{A, m}^{2} \equiv \max \left\{-Z_{5} v^{2}, 0\right\}$.

That is, if $Z_{5}$ is sufficiently large and negative, then the minimal allowed value of $M_{A}^{2}$ is non-zero and positive.

We focus again on the parameter region in the $\left(\widehat{\mu}, \widehat{A}_{t}\right)$ plane, and compute $Z_{5}$ from Eq. (23) using the value of $\tan \beta$ obtained from setting $Z_{6}=0$ in Eq. (20). This allows us to determine the value of $M_{A, c}^{2}$ for each point in the $\left(\widehat{\mu}, \widehat{A}_{t}\right)$ plane. The interpretation of $M_{A, c}^{2}$ is as follows. If $M_{A}^{2}>$ $M_{A, c}^{2}$, then $h$ can be identified as the SM-like Higgs boson with $M_{h} \simeq 125 \mathrm{GeV}$. If $M_{A, m}^{2}<M_{A}^{2}<M_{A, c}^{2}$, then $H$ can be identified as the SM-like Higgs boson with $M_{H} \simeq 125 \mathrm{GeV}$. The corresponding contours of $M_{A, c}$ are exhibited in the three left panels of Fig. 2, which are in one-to-one correspondence with the three left panels of Fig. 1.
As previously noted, the analysis above was based on approximate one-loop formulae given in Eqs. (15)-(17), where only the leading terms proportional to $m_{t}^{2} h_{t}^{2}$ are included. In the exact alignment limit, we identify $Z_{1} v^{2}$ given by Eq. (15) as the squared-mass of the observed SMlike Higgs boson. However, it is well known that Eq. (15) overestimates the value of the radiatively corrected Higgs mass. Remarkably, one can obtain a significantly more accurate result simply by including the leading two-loop radiative corrections proportional to $\alpha_{s} m_{t}^{2} h_{t}^{2}$.

In Ref. [59], it was shown that the dominant part of these two-loop corrections can be obtained from the corresponding one-loop formulae with the following very simple two step prescription. First, we replace

$m_{t}^{4} \ln \left(\frac{M_{S}^{2}}{m_{t}^{2}}\right) \longrightarrow m_{t}^{4}(\lambda) \ln \left(\frac{M_{S}^{2}}{m_{t}^{2}(\lambda)}\right)$,

where $\lambda \equiv\left[m_{t}\left(m_{t}\right) M_{S}\right]^{1 / 2}$,

where $m_{t}\left(m_{t}\right) \simeq 165.6 \mathrm{GeV}$ is the $\overline{\mathrm{MS}}$ top quark mass [60], and the running top quark mass in the one-loop approximation is given by

$m_{t}(\lambda)=m_{t}\left(m_{t}\right)\left[1+\frac{\alpha_{s}}{\pi} \ln \left(\frac{m_{t}^{2}\left(m_{t}\right)}{\lambda^{2}}\right)\right]$.

In our numerical analysis, we take $\alpha_{s}=\alpha_{s}\left(m_{t}\left(m_{t}\right)\right) \simeq$ 0.10826 . Second, when $m_{t}^{4}$ multiplies that threshold corrections due to stop mixing (i.e., the one-loop terms proportional to $X_{t}$ and $Y_{t}$ ), then we make the replacement,

$m_{t}^{4} \longrightarrow m_{t}^{4}\left(M_{S}\right)$,

where

$m_{t}\left(M_{S}\right)=m_{t}\left(m_{t}\right)\left[1+\frac{\alpha_{s}}{\pi} \ln \left(\frac{m_{t}^{2}\left(m_{t}\right)}{M_{S}^{2}}\right)+\frac{\alpha_{S}}{3 \pi} \frac{X_{t}}{M_{S}}\right]$.

Note that the running top-quark mass evaluated at $M_{S}$ includes a threshold correction proportional to $X_{t}$ that enters at the scale of supersymmetry breaking. Here, we only keep the leading contribution to the threshold correction under the assumption that $m_{t} \ll M_{S}$ (a more precise formula can be found in Appendix B of Ref. [59]). The above two step prescription can now be applied to Eqs. (15)-(17), which yields a more accurate expression for the radiatively corrected Higgs mass and the condition for exact alignment without decoupling. Details of this analysis will be presented in a forthcoming work [47].

The end results are summarized below. We have derived analogous expressions to Eqs. (20) and (23) that incorporate the leading two-loop effects at $\mathcal{O}\left(\alpha_{s} h_{t}^{2}\right)$. It is convenient to 
$M_{A, C} \leq 125 \mathrm{GeV} \square M_{A, C} \leq 150 \mathrm{GeV} \square M_{A, c} \leq 200 \mathrm{GeV}$

$\square M_{A, C} \leq 250 \mathrm{GeV} \square M_{A, C} \leq 300 \mathrm{GeV} \square M_{A, C}>300 \mathrm{GeV}$

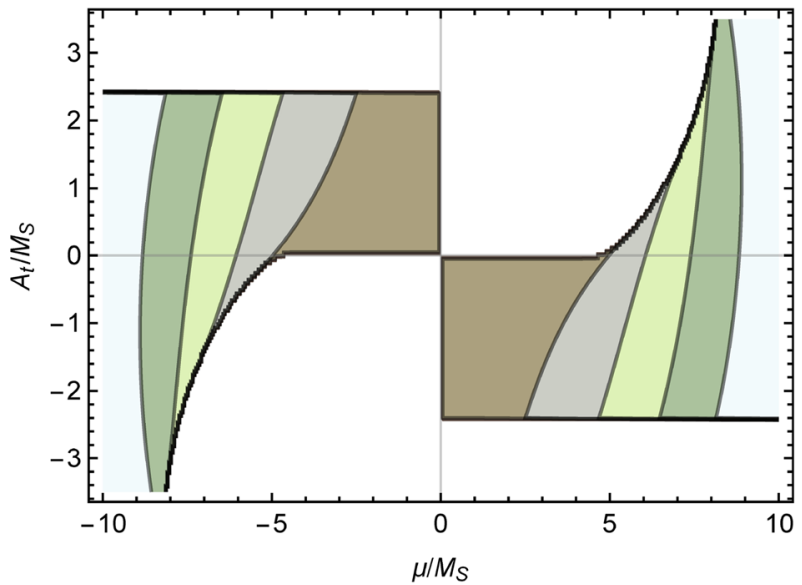

$M_{A, c} \leq 125 \mathrm{GeV} \square M_{A, C} \leq 150 \mathrm{GeV} \square M_{A, C} \leq 200 \mathrm{GeV}$

$\square M_{A, C} \leq 250 \mathrm{GeV} \square M_{A, C} \leq 300 \mathrm{GeV} \square M_{A, C}>300 \mathrm{GeV}$

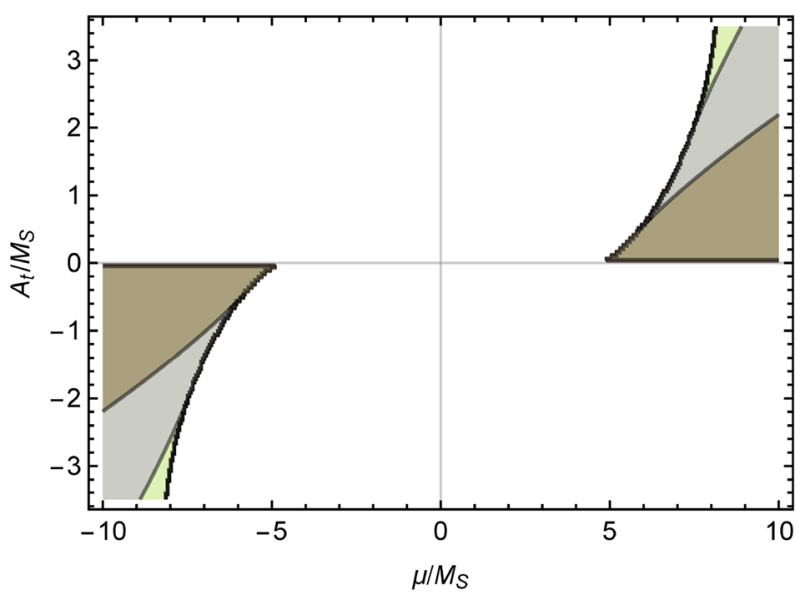

$M_{A, C} \leq 125 \mathrm{GeV} \square M_{A, C} \leq 150 \mathrm{GeV} \square M_{A, c} \leq 200 \mathrm{GeV}$

$\square M_{A, C} \leq 250 \mathrm{GeV} \square M_{A, C} \leq 300 \mathrm{GeV} \square M_{A, C}>300 \mathrm{GeV}$

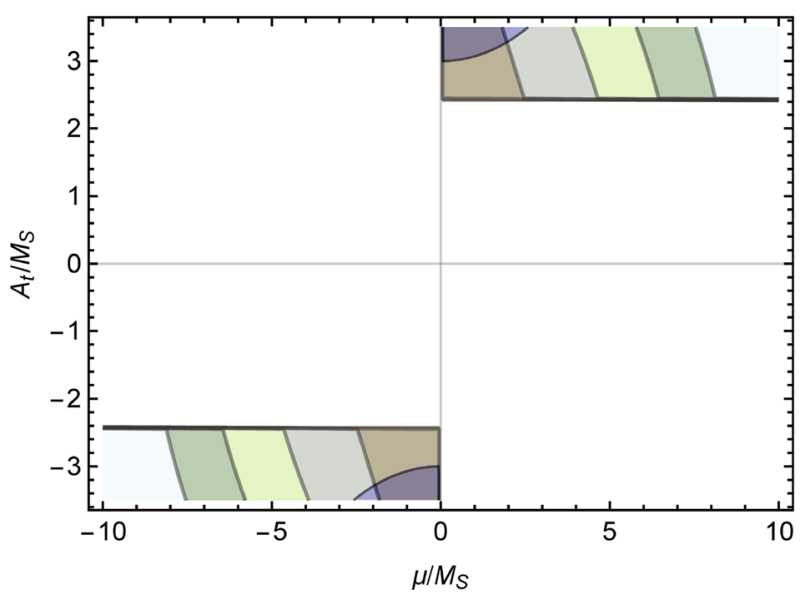

Fig. 2 Critical $M_{A}$ value, $M_{A, c}$, in the exact alignment, indicating the maximal $M_{A}$ value for which the mass hierarchy of the heavy Higgs interpretation is obtained, corresponding to the solutions found in Fig. 1 in the $\left(\mu / M_{S}, A_{t} / M_{S}\right)$ plane. The three left panels exhibit the approx-
$M_{A, C} \leq 125 \mathrm{GeV} \square M_{A, C} \leq 150 \mathrm{GeV} \square M_{A, C} \leq 200 \mathrm{GeV}$

$\square M_{A, C} \leq 250 \mathrm{GeV} \square M_{A, C} \leq 300 \mathrm{GeV} \square M_{A, C} \leq 350 \mathrm{GeV}$

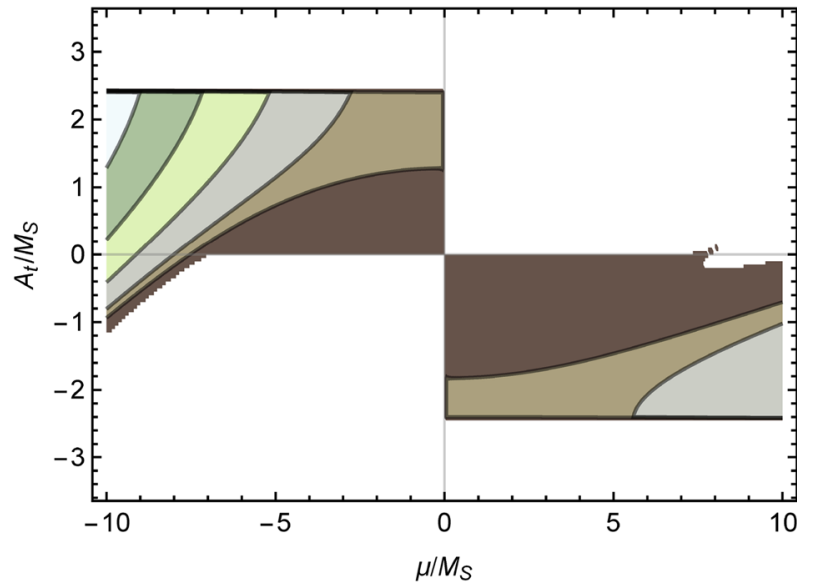

$M_{A, C} \leq 125 \mathrm{GeV} \square M_{A, C} \leq 150 \mathrm{GeV} \square M_{A, C} \leq 200 \mathrm{GeV}$

$\square M_{A, C} \leq 250 \mathrm{GeV} \square M_{A, C} \leq 300 \mathrm{GeV} \square M_{A, C} \leq 350 \mathrm{GeV}$

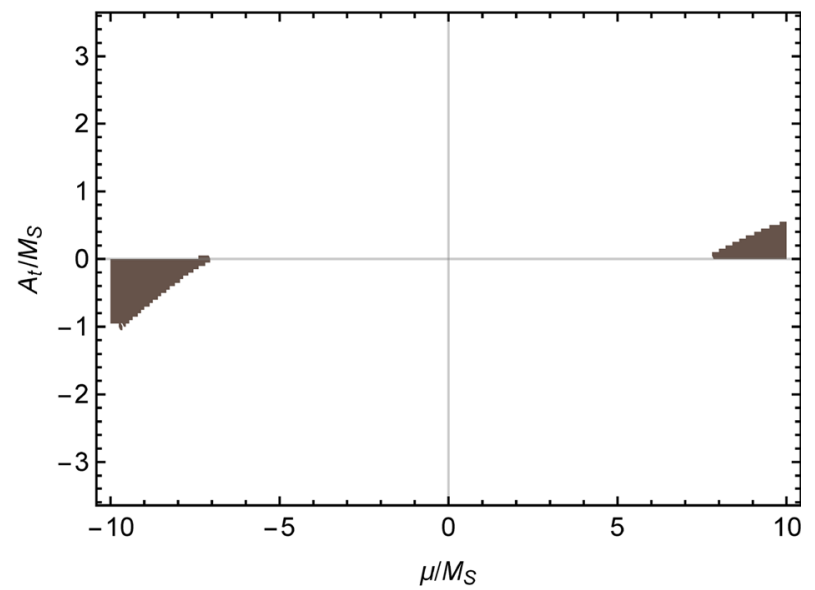

$M_{A, C} \leq 125 \mathrm{GeV} \square M_{A, C} \leq 150 \mathrm{GeV} \square M_{A, C} \leq 200 \mathrm{GeV}$

$\square M_{A, c} \leq 250 \mathrm{GeV} \square M_{A, C} \leq 300 \mathrm{GeV} \square M_{A, C} \leq 350 \mathrm{GeV}$

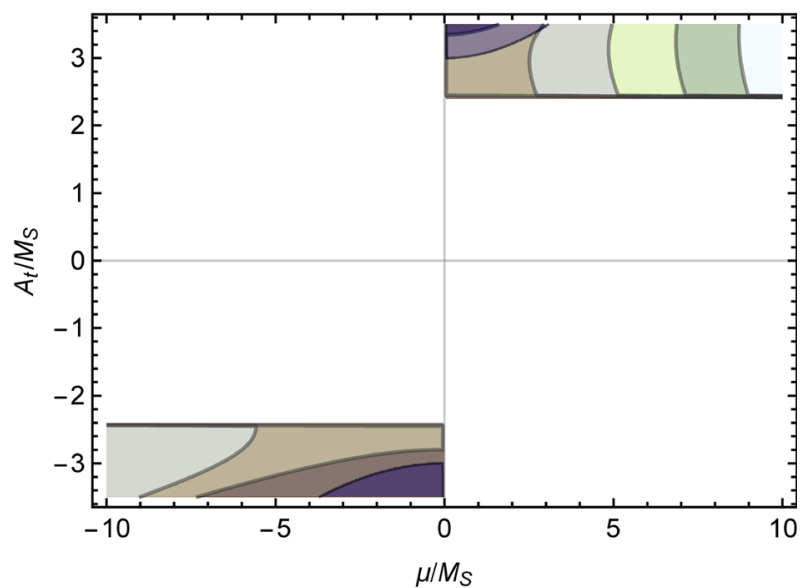

imate one-loop results; the three right panels exhibit the corresponding two-loop improved results. In the overlaid blue regions we have (unstable) values of $\left|X_{t} / M_{S}\right| \geq 3$ 
introduce the following notation,

$C \equiv \frac{3 m_{t}^{4}}{2 \pi^{2} v^{2}}, \quad \bar{\alpha}_{s} \equiv \frac{\alpha_{s}}{\pi}, \quad x_{t} \equiv X_{t} / M_{S}$,

where $m_{t} \equiv m_{t}\left(m_{t}\right)$ is the $\overline{\mathrm{MS}}$ top quark mass, and

$X_{1} \equiv x_{t}^{2}\left(1-\frac{1}{12} x_{t}^{2}\right), \quad X_{5} \equiv x_{t} y_{t}\left(1-\frac{1}{12} x_{t} y_{t}\right)$,

$X_{6} \equiv \frac{1}{2} x_{t}\left(x_{t}+y_{t}\right)-\frac{1}{12} x_{t}^{3} y_{t}$.

Then, the two-loop corrected condition for the exact alignment limit corresponding to $Z_{6}=0$ is given by,

$$
\begin{aligned}
& 2 M_{Z}^{2} s_{\beta}^{2} c_{2 \beta}-\left(Z_{1} v^{2}-M_{Z}^{2} c_{2 \beta}^{2}\right)\left[1+4 \bar{\alpha}_{s}\left(X_{1}-X_{6}\right)\right] \\
& +C\left(X_{1}-X_{6}\right)\left[1+\bar{\alpha}_{s}\left(4 X_{1}+\frac{4}{3} x_{t}\right)\right]=0,
\end{aligned}
$$

which supersedes Eq. (20), and the $\mathcal{O}\left(\alpha_{s}\right)$ correction to Eq. (23) is given by,

$$
\begin{aligned}
Z_{5} v^{2}= & M_{Z}^{2}\left(1+c_{2 \beta}\right)+\frac{C\left(X_{5}-X_{6}\right)}{\tan ^{2} \beta} \\
& \times\left\{1+4 \bar{\alpha}_{s}\left(X_{6}+\frac{1}{3} x_{t}-2 s_{\beta}^{2} c_{2 \beta} C^{-1} M_{Z}^{2}\right)\right\} .
\end{aligned}
$$

One can now define two-loop improved versions of $M_{A, c}^{2}$ and $M_{A, m}^{2}$ [cf. Eqs. (24) and (25)].

In the right panels of Figs. 1 and 2, we plot the twoloop improved versions of the corresponding one-loop results shown in the left panels. There are a few notable changes, which we now discuss. First, in our scan of the $\left(\widehat{\mu}, \widehat{A}_{t}\right)$ plane, we have observed numerically that there is a new solution to the alignment condition [cf. Eq. (32)] that is unrelated to the solutions found in the one-loop analysis. However, this solution always corresponds to a value of $\left|X_{t}\right|>3 M_{S}$, which lies outside our region of interest. Henceforth, we simply discard this possibility. What remains are solutions that can be identified as the two-loop corrected versions of the oneloop results obtained above. The right panels of Fig. 1 exhibit the remaining real positive $\tan \beta$ solutions of Eq. (32).

We can now see the effects of including the leading $\mathcal{O}\left(\alpha_{s} h_{t}^{2}\right)$ corrections. The regions where positive solutions to Eq. (32) exist shown in the right panels of Fig. 1 have shrunk somewhat as compared to the corresponding positive solutions to Eq. (20) shown in the left panels of Fig. 1. For example, only one positive solution for $\tan \beta$ exists for large $\widehat{\mu}$ and $\widehat{A}_{t}$ in the two-loop approximation, whereas three positive solutions exist in the one-loop approximation. Using the values of $\tan \beta$ found in the right panels of Fig. 1, one can now produce the corresponding two-loop corrected plots shown in the right panels of Fig. 2. The qualitative features of the one-loop and two-loop results are similar, after taking note of the slightly smaller regions in which positive solutions for $\tan \beta$ exist in the two-loop approximation.

One new feature of the two-loop approximation not yet emphasized is that we must now carefully define the input parameters $\mu$ and $A_{t}$. In the above formulae and plots we interpret these parameters as $\overline{\mathrm{MS}}$ parameters. However, it is often more convenient to re-express these parameters in terms of on-shell parameters. In Ref. [59], the following expression was obtained for the on-shell squark mixing parameter $X_{t}^{\mathrm{OS}}$ in terms of the $\overline{\mathrm{MS}}$ squark mixing parameter $X_{t}$, where only the leading $\mathcal{O}\left(\alpha_{S}\right)$ corrections are kept,

$X_{t}^{\mathrm{OS}}=X_{t}-\frac{\alpha_{s}}{3 \pi} M_{S}\left[8+\frac{4 X_{t}}{M_{S}}-\frac{X_{t}^{2}}{M_{S}^{2}}-\frac{3 X_{t}}{M_{S}} \ln \left(\frac{m_{t}^{2}}{M_{S}^{2}}\right)\right]$

Since the on-shell and $\overline{\mathrm{MS}}$ versions of $\mu$ are equal at this level of approximation, we also have

$A_{t}^{\mathrm{OS}}=X_{t}^{\mathrm{OS}}+\frac{\mu}{\tan \beta}$.

A more detailed examination of the above results, when expressed in terms of the on-shell parameters, will be treated in Ref. [47].

The approximations employed in the section capture some of the most important radiative corrections relevant for analyzing the alignment limit of the MSSM. However, it is important to appreciate what has been left out. First, higherorder corrections beyond $\mathcal{O}\left(\alpha_{s} h_{t}^{2}\right)$ are known to be relevant (see, e.g., Ref. [61]). In particular the $\mathcal{O}\left(h_{t}^{4}\right)$ corrections are in magnitude roughly $20 \%$ of the $\mathcal{O}\left(\alpha_{s} h_{t}^{2}\right)$ corrections, and enter with a different sign, thus leading to potentially nonnegligible corrections to the approximate two-loop results obtained above. On more general grounds, the analysis of this section ultimately corresponds to a renormalization of $\cos (\beta-\alpha)$, which governs the tree-level couplings of the Higgs boson and its departure from the alignment limit. However, radiative corrections also contribute other effects that modify Higgs production cross sections and branching ratios. It is well-known that for $M_{A} \ll M_{S}$, the effective low-energy theory below the scale $M_{S}$ is a general two Higgs doublet model with the most general Higgs-fermion Yukawa couplings. These include the so-called wrong-Higgs couplings of the MSSM $[62,63]$, which ultimately are responsible for the $\Delta_{b}$ and $\Delta_{\tau}$ corrections that can significantly modify the coupling of the Higgs boson to bottom quarks and tau leptons. ${ }^{4}$ The implication of these couplings will be briefly reviewed in Sect. 2.3. In addition, integrating out heavy SUSY particles at the scale $M_{S}$ can generate higher dimensional operators that can also modify Higgs production cross sections and branching ratios [65]. None of these effects are accounted for in the analysis presented in this section.

\footnotetext{
${ }^{4}$ For a review of these effects and a guide to the original literature, see Ref. [64].
} 


\subsection{Implications of the wrong-Higgs couplings}

At tree-level, the Higgs-fermion Yukawa couplings follow the Type-II pattern $[53,66]$ of the two-Higgs doublet model (2HDM), in which the hypercharge -1 Higgs doublet field $H_{D}$ couples exclusively to right-handed down-type fermions and the hypercharge +1 Higgs doublet field $H_{U}$ couples exclusively to right-handed up-type fermions. When radiative corrections are included, the so-called wrongHiggs Yukawa couplings are induced by supersymmetrybreaking effects, in which $H_{D}^{*}$ couples to right-handed uptype fermions and $H_{U}^{*}$ couples to right-handed down-type fermions. We shall denote by $M_{\text {SUSY }}$ a generic scale that characterizes the size of supersymmetric mass parameters. In the limit where $M_{Z}, M_{A} \ll M_{\mathrm{SUSY}}$, the radiatively-corrected Higgs-quark Yukawa couplings can be summarized by an effective Lagrangian, ${ }^{5}$

$$
\begin{aligned}
-\mathscr{L}_{\text {eff }}= & \epsilon_{i j}\left[\left(h_{b}+\delta h_{b}\right) \bar{b}_{R} H_{D}^{i} Q_{L}^{j}+\left(h_{t}+\delta h_{t}\right) \bar{t}_{R} Q_{L}^{i} H_{U}^{j}\right] \\
& +\Delta h_{t} \bar{t}_{R} Q_{L}^{k} H_{D}^{k *}+\Delta h_{b} \bar{b}_{R} Q_{L}^{k} H_{U}^{k *}+\text { h.c., }
\end{aligned}
$$

which yields a modification of the tree-level relations between $h_{t}, h_{b}$ and $m_{t}, m_{b}$ as follows [64,67-74]:

$$
\begin{aligned}
m_{b} & =\frac{h_{b} v}{\sqrt{2}} \cos \beta\left(1+\frac{\delta h_{b}}{h_{b}}+\frac{\Delta h_{b} \tan \beta}{h_{b}}\right) \\
& \equiv \frac{h_{b} v}{\sqrt{2}} \cos \beta\left(1+\Delta_{b}\right), \\
m_{t} & =\frac{h_{t} v}{\sqrt{2}} \sin \beta\left(1+\frac{\delta h_{t}}{h_{t}}+\frac{\Delta h_{t} \cot \beta}{h_{t}}\right) \\
& \equiv \frac{h_{t} v}{\sqrt{2}} \sin \beta\left(1+\Delta_{t}\right) .
\end{aligned}
$$

The dominant contributions to $\Delta_{b}$ are $\tan \beta$-enhanced, with $\Delta_{b} \simeq\left(\Delta h_{b} / h_{b}\right) \tan \beta$. Moreover, in light of our assumption that $M_{Z}, M_{A} \ll M_{\text {SUSY, }}$, it follows that $\delta h_{b} \sim$ $\mathcal{O}\left(M_{Z}^{2} / M_{\text {SUSY }}^{2}\right)$ is suppressed, whereas $\Delta h_{b}$ does not decouple. This non-decoupling can be explained by the fact that $\Delta h_{b}$ arises from the radiatively-generated wrong-Higgs couplings. Below the scale $M_{\text {SUSY }}$, the effective low energy theory is the 2HDM which contains the most general set of Higgs-fermion Yukawa couplings allowed by gauge invariance, and is no longer restricted to be of Type-II [62]. Similarly, $\delta h_{t} \sim \mathcal{O}\left(M_{Z}^{2} / M_{\text {SUSY }}^{2}\right)$ is suppressed, whereas $\Delta h_{t}$ does not decouple. However, $\Delta_{t}$ is not $\tan \beta$-enhanced and thus yields only small corrections to the Higgs boson couplings to fermions in the parameter regime of interest (i.e., where $\tan \beta \gtrsim 1$ ).

\footnotetext{
5 For simplicity, we ignore the couplings to first and second generation fermions. We also neglect weak isospin breaking effects that distinguish between the coupling of neutral and charged Higgs scalars.
}

In the parameter regime where $M_{Z}, M_{A} \ll M_{\text {SUSY }}[67-$ $69,73,75]$,

$$
\begin{aligned}
\Delta_{b}= & {\left[\frac{2 \alpha_{s}}{3 \pi} \mu m_{\tilde{g}} I\left(m_{\tilde{b}_{1}}, m_{\tilde{b}_{2}}, m_{\tilde{g}}\right)\right.} \\
& \left.+\frac{h_{t}^{2}}{16 \pi^{2}} \mu A_{t} I\left(m_{\tilde{t}_{1}}, m_{\tilde{t}_{2}}, \mu\right)\right] \tan \beta \\
& +\mathcal{O}\left(\frac{M_{Z}^{2}}{M_{\mathrm{SUSY}}^{2}}\right),
\end{aligned}
$$

where $m_{\tilde{g}}$ is the gluino mass, $m_{\tilde{b}_{1,2}}$ and $m_{\tilde{t}_{1,2}}$ are the bottom and top squark masses, respectively, and smaller electroweak corrections have been ignored. The loop integral $I\left(a^{2}, b^{2}, c^{2}\right)$ is given by

$$
\begin{aligned}
& I(a, b, c) \\
& \quad=\frac{a^{2} b^{2} \ln \left(a^{2} / b^{2}\right)+b^{2} c^{2} \ln \left(b^{2} / c^{2}\right)+c^{2} a^{2} \ln \left(c^{2} / a^{2}\right)}{\left(a^{2}-b^{2}\right)\left(b^{2}-c^{2}\right)\left(a^{2}-c^{2}\right)},
\end{aligned}
$$

Note that

$I(a, a, c)=\frac{a^{2}-c^{2}+c^{2} \ln \left(c^{2} / a^{2}\right)}{\left(c^{2}-a^{2}\right)^{2}}$,

and $I(a, a, a)=1 /\left(2 a^{2}\right)$. Thus, in the limit in which all supersymmetric parameters appearing in Eq. (39) are all very large, of $\mathcal{O}\left(M_{\text {SUSY }}\right)$, we see that $\Delta_{b} \simeq\left(\Delta h_{b} / h_{b}\right) \tan \beta$ does not decouple, as previously advertised.

From Eq. (36) we can obtain the couplings of the physical Higgs bosons to third generation fermions. The resulting interaction Lagrangian is of the form,

$$
\begin{aligned}
\mathscr{L}_{\text {int }}= & -\sum_{q=t, b, \tau}\left[g_{h q \bar{q}} q \bar{q} h+g_{H q \bar{q}} q \bar{q} H-i g_{A q \bar{q}} \bar{q} \gamma_{5} q A\right] \\
& +\left[g_{H^{-} t \bar{b}} \bar{b} t H^{-}+\text {h.c. }\right] .
\end{aligned}
$$

Expressions for the Higgs couplings to the third generation quarks can be found in Ref. [64]. In particular, the charged Higgs coupling to the third generation quarks is noteworthy. It is convenient to write the approximate one-loop corrected $H^{-} t \bar{b}$ coupling in the following form,

$$
\begin{aligned}
& g_{H^{-} t \bar{b}} \simeq\left[h_{t} \cos \beta\left(1+\frac{\delta h_{t}}{h_{t}}\right)-\Delta h_{t} \sin \beta\right] P_{R} \\
& +\frac{\sqrt{2} m_{b}}{v} \tan \beta\left[1+\frac{1}{\left(1+\Delta_{b}\right) \sin ^{2} \beta}\left(\frac{\delta h_{b}}{h_{b}}-\Delta_{b}\right)\right] P_{L},
\end{aligned}
$$

where $P_{R, L} \equiv \frac{1}{2}\left(1 \pm \gamma_{5}\right)$.

One of the important constraints on the MSSM Higgs sector is derived from the decay rate for $b \rightarrow s \gamma$ due to the presence of one loop diagrams involving a charged Higgs boson. At large $\tan \beta$, it is important to incorporate SUSY 
corrections to the charged Higgs couplings to quarks ${ }^{6}$ in the computation of $\mathrm{BR}(b \rightarrow s \gamma)$ [76]. Including the radiatively corrected $H^{+} \bar{t} b$ and $H^{-} t \bar{s}$ couplings using Eq. (43), suitably generalized to include intergenerational quark mixing, and taking $\tan \beta \gg 1$,

$$
\frac{\mathrm{BR}(b \rightarrow s \gamma)_{\mathrm{MSSM}, H^{ \pm}}}{\mathrm{BR}(b \rightarrow s \gamma)_{2 \mathrm{HDM}-\mathrm{II}}} \simeq \frac{1}{1+\Delta_{b}}\left[1-\frac{\Delta h_{t}}{h_{t}} \tan \beta\right],
$$

after comparing the result obtained from the contribution of the charged Higgs loop in the MSSM, including the leading SUSY radiative corrections to the charged Higgs-fermion couplings, to the corresponding results of the 2HDM with Type-II Yukawa couplings. In Eq. (44), $\Delta_{b}$ is given by Eq. (39) and $\Delta h_{t}$ is given by [76]

$$
\begin{aligned}
\frac{\Delta h_{t}}{h_{t}} \simeq & \frac{2 \alpha_{s}}{3 \pi} \mu m_{\tilde{g}}\left[\cos ^{2} \theta_{\tilde{t}} I\left(m_{\tilde{s}_{L}}, m_{\tilde{t}_{2}}, m_{\tilde{g}}\right)\right. \\
& \left.+\sin ^{2} \theta_{\tilde{t}} I\left(m_{\tilde{s}_{L}}, m_{\tilde{t}_{1}}, m_{\tilde{g}}\right)\right],
\end{aligned}
$$

where $m_{\widetilde{s}_{L}}$ is the mass of the SUSY partner of the left-handed strange quark, $\theta_{\tilde{t}}$ is the $\tilde{t}_{L}-\widetilde{t}_{R}$ mixing angle [77], and $I$ is defined in Eq. (40). Once again, the non-decoupling behavior of $\Delta h_{t}$ is evident in the limit in which all supersymmetric parameters appearing in Eq. (45) are of $\mathcal{O}\left(M_{\text {SUSY }}\right)$. As previously emphasized, the non-decoupling properties $\Delta_{b}$ and $\Delta h_{t}$ arise due to the wrong-Higgs Yukawa couplings, and are responsible for the significance of the deviation from Type-II behavior of the two-Higgs doublet sector of the MSSM.

\section{Parameter sampling, observables and constraints}

\subsection{Sampling of the parameter space}

We sample the $p M S S M 8$ parameter space with uniformly distributed random values in the eight input parameters. Scans are performed separately for the light Higgs and heavy Higgs interpretation of the observed Higgs signal (see below for details) over the parameter ranges given in Table 1. Besides the scan parameters listed in Table 1, the remaining MSSM parameters are chosen as described in Sect. 2.1.

In both cases, we start with $\mathcal{O}\left(10^{7}\right)$ randomly sampled points in the ranges given in Table 1 and identify interesting regions where either $h$ or $H$ has a mass close to the observed signal at $125 \mathrm{GeV}$ (i.e. we select points with $\left.M_{h / H} \in[120,130] \mathrm{GeV}\right)$ and the global $\chi^{2}$ function is low, see Sect. 3.2 for details on how the global $\chi^{2}$ function is evaluated. In a second step we perform dedicated smaller scans over more restricted parameter ranges in order to obtain high

${ }^{6}$ By including the radiative corrections via Eq. (43), we are effectively incorporating the leading two-loop contributions to the decay matrix element for $b \rightarrow s \gamma$ induced by SUSY vertex corrections. sampling densities in the interesting regions of the parameter space.

The choices of the parameter ranges for the light Higgs and heavy Higgs case differ in particular for $M_{A}$ and $\tan \beta$, where the ranges in the heavy Higgs case are quite restricted. This is because $M_{H} \sim 125 \mathrm{GeV}$ can only be obtained in a rather small region of the parameter space, and a high sampling density in this region is desired. Furthermore, while we scan the third generation squark masses, $M_{\tilde{q}_{3}}$, up to $5 \mathrm{TeV}$ in the light Higgs case, we restrict $M_{\tilde{q}_{3}}$ to be at most $1.5 \mathrm{TeV}$ in the heavy Higgs case. As mentioned before, the SM-like Higgs boson mass can be lifted to the observed value of $\sim 125 \mathrm{GeV}$ by radiative corrections from either a large stop mass scale, $M_{S}$, or from a large stop mixing parameter, $X_{t}$. We consider a larger $M_{\tilde{q}_{3}}$ range in the light Higgs case in order to allow for solutions with small to moderate $X_{t}, \mu$ and $A_{t}$ values. In contrast, in the heavy Higgs case, the SM-like properties can only be obtained in the alignment limit (without decoupling) which already requires large values of $\mu / M_{S}$ and/or $A_{t} / M_{S}$ (see Sect. 2.2), and we restrict ourselves to $M_{\tilde{q}_{3}}<1.5 \mathrm{TeV}$ in this case. Lastly, the choice of the scanning range in the Higgsino mass parameter, $\mu$, differs in the two cases. In the light Higgs case we restrict $|\mu| \leq 3 M_{\tilde{q}_{3}}$, thus allowing $\mu \lesssim 15 \mathrm{TeV}$ for very large third generation squark masses $M_{\tilde{q}_{3}} \sim 5 \mathrm{TeV}$. Parameter points with more extreme values of $\left|\mu / M_{\tilde{q}_{3}}\right|$ beyond $\sim 3$ often face severe constraints from vacuum stability requirements [78-86] (for a recent analysis see also Ref. [87]). Nevertheless, in the heavy Higgs case we include such more extreme values of $\left|\mu / M_{\tilde{q}_{3}}\right|$ and do not impose a specific upper limit on this ratio. As we discussed in Sect. 2.2, $\left|\mu / M_{S}\right|$ greatly influences the $\tan \beta$ value where the alignment limit occurs as well as the critical $M_{A}$ value that indicates the crossover of the light and heavy Higgs case mass hierarchies. As we will see, a large ratio $\mu / M_{\tilde{q}_{3}}$ will be crucial to obtain an acceptable fit of the heavy Higgs to the observed Higgs signal. We will comment on the fit outcome in the case where the requirement $\left|\mu / M_{\tilde{q}_{3}}\right| \leq 3$ is imposed.

Table 1 Ranges used for the free parameters in the $p M S S M 8$ scan

\begin{tabular}{llllll}
\hline Parameter & \multicolumn{2}{l}{ Light Higgs case } & & \multicolumn{2}{c}{ Heavy Higgs case } \\
\cline { 2 - 3 } \cline { 5 - 6 } & Minimum & Maximum & & Minimum & Maximum \\
\hline$M_{A}(\mathrm{GeV})$ & 90 & 1000 & & 90 & 200 \\
$\tan \beta$ & 1 & 60 & 1 & 20 \\
$M_{\tilde{q}_{3}}(\mathrm{GeV})$ & 200 & 5000 & & 200 & 1500 \\
$M_{\tilde{\ell}_{3}}(\mathrm{GeV})$ & 200 & 1000 & & 200 & 1000 \\
$M_{\tilde{\ell}_{1,2}}(\mathrm{GeV})$ & 200 & 1000 & & 200 & 1000 \\
$\mu(\mathrm{GeV})$ & $-3 M_{\tilde{q}_{3}}$ & $3 M_{\tilde{q}_{3}}$ & & -5000 & 5000 \\
$A_{f}(\mathrm{GeV})$ & $-3 M_{\tilde{q}_{3}}$ & $3 M_{\tilde{q}_{3}}$ & & $-3 M_{\tilde{q}_{3}}$ & $3 M_{\tilde{q}_{3}}$ \\
$M_{2}(\mathrm{GeV})$ & 200 & 500 & & 200 & 500 \\
\hline
\end{tabular}


In our scans we allow both signs of the Higgsino mass parameter $\mu$. The sign of the SUSY contributions to the anomalous magnetic moment of the muon, $(g-2)_{\mu}$, is given by the sign of $\mu$, thus the negative $\mu$ branch is significantly disfavored in the light of this observable, receiving a $\chi^{2}$ penalty like the SM or higher, depending on the mass scale of the relevant SUSY particles. In this work we will therefore present fit results where $(g-2)_{\mu}$ is either included or excluded from the global $\chi^{2}$ function, see below for details.

In addition to the eight $p M S S M 8$ scan parameters we sample the top quark pole mass from a Gaussian distribution with $m_{t}=173.34 \pm 0.76 \mathrm{GeV}$ [88], using a cutoff at $\pm 2 \sigma$. Effects from other parametric uncertainties of SM quantities are estimated to be small and therefore neglected in this analysis.

We calculate the SUSY particle spectrum and the MSSM Higgs masses using FeynHiggs (version 2.11.2) ${ }^{7}$ [61, 90-94] and estimate the remaining theoretical uncertainty (e.g. from unknown higher-order corrections) in the Higgs mass calculation to be $3 \mathrm{GeV}$ [61]. Following Refs. [19,35], we demand that all points fulfill a $\mathbf{Z}$-matrix criterion, ||$Z_{k 1}^{2 \mathrm{~L}}|-| Z_{k 1}^{1 \mathrm{~L}}|| /\left|Z_{k 1}^{1 \mathrm{~L}}\right|<0.25$ (with $k=1$ (2) in the light (heavy) Higgs case), in order to ensure a reliable and stable perturbative behavior in the calculation of propagator-type contributions in the MSSM Higgs sector. ${ }^{8}$ In the Feynmandiagrammatic approach of FeynHiggs all model parameters (except for $\tan \beta$, which is a $\overline{\mathrm{DR}}$ parameter defined at the scale $m_{t}$ ) are defined in the on-shell (OS) renormalization scheme, which we adopt for the definition of our fit parameters [cf. (3)].

\subsection{Observables}

In our scan we take into account the following experimental measurements (we denote all experimental measurements with a hat, while unhatted quantities correspond to the model predictions of the respective quantity):

\section{- Higgs boson mass:}

We use the combined result from the ATLAS and CMS Higgs mass measurements [3],

$$
\hat{M}_{H}=(125.09 \oplus 0.21 \text { (stat.) } \oplus 0.11 \text { (syst.)) } \mathrm{GeV}
$$

where we linearly combine the uncertainties. In our pMSSM 8 scans, where the measured Higgs mass corresponds to either the light Higgs or the heavy Higgs mass,

\footnotetext{
7 Recent updates in the Higgs boson mass calculations [89] lead to a downward shift in $M_{h}$, in particular for large values of $X_{t} / M_{S}$. These changes range within the estimated uncertainties and should not have a drastic impact on our analysis.

${ }^{8}$ The $\mathbf{Z}$-matrix is defined in Ref. [92].
}

we linearly add the theoretical Higgs mass uncertainty of $3 \mathrm{GeV}$. Thus, the total mass uncertainty in the MSSM case is $\sigma_{\hat{M}_{H}}=3.32 \mathrm{GeV}$.

- Higgs signal rates:

We employ the public code Higgs Signals-1 . 4 . 0 [17, $95,96]$ to evaluate a $\chi^{2}$ value, $\chi_{\mathrm{HS}}^{2}$, for the compatibility of the pMSSM 8 predictions with rate measurements in 85 different Higgs signal channels from the LHC experiments ATLAS and CMS, as well as the Tevatron experiments $\mathrm{CDF}$ and $\mathrm{D} \emptyset$. A detailed list of all Higgs rate observables is given in Appendix B. HiggsSignals takes into account the correlations among major systematic uncertainties, including the uncertainties of the integrated luminosity and the theoretical uncertainties for the cross section and branching ratio predictions for a SM Higgs boson. It furthermore takes into account a potential overlap of signals from nearby Higgs bosons by simply adding the signal rates if the mass difference of the Higgs bosons is less than the experimental mass resolution of the search channel. ${ }^{9}$ This feature is of relevance for the heavy Higgs interpretation where all three neutral Higgs bosons can be within the mass range $\sim(100-150) \mathrm{GeV}$. For instance, the mass resolution of the $H_{\mathrm{SM}} \rightarrow \tau^{+} \tau^{-}$ analyses is typically assumed to be $\sim 25 \mathrm{GeV}$, thus the $\tau^{+} \tau^{-}$signal rates of Higgs bosons within the above mass range will potentially be added by HiggsSignals.

The Higgs production cross sections are evaluated, both in the MSSM and the SM, with the code FeynHiggs (version 2.11.2) [61,90-92]. This includes an implementation of the SM cross sections of the LHC Higgs cross section working group (LHCHXSWG) [98-100] (using the $g g \rightarrow H$ cross section prediction from Refs. [101,102]). The MSSM Higgs production cross sections are calculated in the effective coupling approximation [103]. More details on the calculation of the production cross section in the various channels can be found in Refs. [103,104]. The Higgs decay widths are also calculated with FeynHiggs, including the full one-loop corrections for the Higgs decay to fermions and leading higher-order contributions [105, 106].

- Low energy observables (LEOs):

We include the rare $B$ meson decays $B \rightarrow X_{S} \gamma\left(X_{s}\right.$ represents any hadronic system containing a strange quark), $B_{s} \rightarrow \mu^{+} \mu^{-}$and $B^{+} \rightarrow \tau^{+} \nu_{\tau}$, which have small branching ratios in the SM, being either loop or helicity suppressed. In SUSY, however, they can be mediated through SUSY particles and/or charged Higgs bosons, which can

\footnotetext{
${ }^{9}$ Interference effects can be incorporated in this context using the method developed in Ref. [97]. Since in our analysis non-negligible interference contributions only occur between the $\mathcal{C P}$-even states $h$ and $H$ in the parameter regions where they are nearly mass-degenerate, we neglect these effects here.
} 
Table 2 The experimental values and SM theory predictions for the low-energy observables (LEOs) that are used in the $p M S S M 8$ scan. The last column lists additional uncertainties intrinsic to the MSSM predictions

\begin{tabular}{llll}
\hline Observable & Experimental value & SM value & MSSM uncertainty \\
\hline $\mathrm{BR}\left(B \rightarrow X_{s} \gamma\right)$ & $(3.43 \pm 0.21 \pm 0.07) \times 10^{-4}[114]$ & $(3.40 \pm 0.22) \times 10^{-4}$ & $\pm 0.15 \times 10^{-4}$ \\
$\mathrm{BR}\left(B_{S} \rightarrow \mu^{+} \mu^{-}\right)$ & $(2.8 \pm 0.7) \times 10^{-9}[117]$ & $(3.54 \pm 0.2) \times 10^{-9}$ & - \\
$\mathrm{BR}\left(B^{+} \rightarrow \tau^{+} \nu_{\tau}\right)$ & $(9.1 \pm 1.9 \pm 1.1) \times 10^{-5}[119,120]$ & $(8.09 \pm 0.7) \times 10^{-5}$ & - \\
$\delta a_{\mu}$ & $(30.2 \pm 9.0) \times 10^{-10}[124-126]$ & - & - \\
$M_{W}$ & $(80.385 \pm 0.015) \mathrm{GeV}[133,134]$ & $(80.358 \pm 0.007) \mathrm{GeV}$ & $\pm 0.003 \mathrm{GeV}$ \\
\hline
\end{tabular}

give sizable contributions. Thus these observables feature a high sensitivity to physics beyond the Standard Model (BSM). ${ }^{10}$

We evaluate the SM prediction - using a top mass value of $m_{t}=173.34 \mathrm{GeV}$ and a Higgs mass value of $M_{H}^{\mathrm{SM}}=$ 125.09 GeV as input - and MSSM predictions for these flavor observables with the public code SuperIso (version 3.5) [111-113]. These predictions are listed besides the latest (combinations of) experimental measurements in Table 2. For BR $\left(B \rightarrow X_{s} \gamma\right)$ we use the current world average of Ref. [114]. Here we assign an additional uncertainty on the MSSM prediction for $\operatorname{BR}\left(B \rightarrow X_{s} \gamma\right)$ of $\pm 0.15 \times 10^{-4}[115,116]$. The process $B_{s} \rightarrow \mu^{+} \mu^{-}$was observed for the first time by LHCb and CMS [117] and recently also by ATLAS [118]. ${ }^{11}$ For $B^{+} \rightarrow \tau^{+} \nu_{\tau}$ we use a Belle combination of measurements using hadronic and semi-leptonic tagging methods with a combined significance of $4.6 \sigma[119,120]$. For all observables in Table 2, theoretical and experimental uncertainties are combined linearly.

The anomalous magnetic moment of the muon, $a_{\mu}=$ $\frac{1}{2}(g-2)_{\mu}$, comprises another very sensitive low energy probe of BSM physics [121-123]. The experimentally observed value exhibits a very persistent deviation from

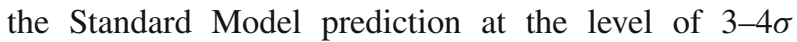
[124-126]. We obtain the MSSM contribution to the anomalous magnetic moment of the muon from SuperIso, which includes the one-loop result [127] as well as leading two-loop contributions [128-131]. We cross-checked the SuperIso result with results from FeynHiggs and found good agreement.

Besides the flavor observables and $a_{\mu}$, we also include the MSSM prediction of the $W$ boson mass into our fit. The SM value for $M_{W}$ shows a $1.8 \sigma$ deviation [132] from the latest experimental value $[133,134]$. Our MSSM evalua-

\footnotetext{
10 We do not include the $B \rightarrow D^{(*)} \tau^{-} \bar{\nu}_{\tau}$ measurements [107-109], which show some tension with respect to the SM prediction. For an explanation within the MSSM (requiring a mass degeneracy between the lightest chargino and neutralino), see [110].

11 The value used in this work does not include the ATLAS measurement yet.
}

tion of $M_{W}$ follows Refs. [132,135] and includes, besides the most advanced SM calculation, the full SUSY oneloop contributions as well as leading SUSY two-loop contributions. The uncertainties from unknown higher-order corrections have been estimated to be around $4 \mathrm{MeV}$ in the SM [136] and somewhat larger $(\sim(4-9) \mathrm{MeV})$ in the MSSM [137,138], depending on the SUSY mass scale. The main parametric uncertainty on $M_{W}$ stems from the top quark mass and does not need to be included in our $\chi^{2}$ evaluation because we vary $m_{t}$ within its $2 \sigma$ uncertainty in the scan. The remaining parametric uncertainties from $M_{Z}$ and $\Delta \alpha_{\text {had }}$ are $\sim 3 \mathrm{MeV}$. Combining these two sources of theoretical uncertainties linearly we estimate $10 \mathrm{MeV}$ for the MSSM uncertainty and $7 \mathrm{MeV}$ for the SM uncertainty.

From these observables and their predictions we evaluate for every parameter point in the scan the global $\chi^{2}$ function

$$
\begin{aligned}
\chi^{2}= & \frac{\left(M_{h, H}-\hat{M}_{H}\right)^{2}}{\sigma_{\hat{M}_{H}}^{2}} \\
& +\chi_{\mathrm{HS}}^{2}+\sum_{i=1}^{n_{\mathrm{LEO}}} \frac{\left(O_{i}-\hat{O}_{i}\right)^{2}}{\sigma_{i}^{2}}-2 \ln \mathcal{L}_{\text {limits }} .
\end{aligned}
$$

As mentioned above, we denote all experimental measurements with a hat. Unhatted quantities correspond to the model predictions of the respective quantity. The sum over the low energy observables $O_{i}$ runs over the five observables mentioned above. The last term, $-2 \ln \mathcal{L}_{\text {limits }}$, denotes the contribution from Higgs search limits at LEP and LHC, for which the likelihood information about the level of exclusion is available. Details will be given below in Sect. 3.3.

The total number of degrees of freedom, $v$, is given by the number of observables, $n_{\mathrm{obs}}$, minus the number of scan parameters, $n_{\text {para }}$. We count every observable and constraint that contributes to the global $\chi^{2}$ function, Eq. (47), to $n_{\mathrm{obs}}$, thus we have in total $n_{\mathrm{obs}}=93$ if all observables are included in the fit. In the SM we have only one free parameter ( $n_{\text {para }}=$ 1 ), namely the Higgs mass, whereas in both MSSM cases we have eight fit parameters $\left(n_{\text {para }}=8\right)$. 


\subsection{Constraints}

- Exclusion limits from Higgs collider searches: For every scan point we test the neutral and charged Higgs bosons against the exclusion limits from Higgs searches at the LEP, Tevatron and LHC experiments by employing the public computer code HiggsBounds-4 . 2 . 1 [23, 139-142]. HiggsBounds determines for each model parameter point the most sensitive exclusion limit, based on the expected exclusion limit given by the experiments. It then judges whether the parameter point is excluded at the $95 \%$ C.L. by comparing the signal prediction against the observed exclusion limit from the most sensitive analysis. In this way, the quoted C.L. of the limit is preserved even though many different Higgs analyses are considered at the same time.

Besides the hard cut imposed by testing the parameter points at the standard 95\% C.L. limit, HiggsBounds enables us to obtain a likelihood value for the model exclusion by LEP Higgs searches [13], as well as by the CMS search for non-standard Higgs bosons decaying into $\tau$ lepton pairs $[143,144]$ (see Ref. [23] for details). While the LEP Higgs searches are only relevant in the heavy Higgs case, i.e. where the heavier Higgs state is the SM-like Higgs boson at $125 \mathrm{GeV}$, the CMS search yields important constraints in either case, and in particular at larger values of $\tan \beta$. Each of these likelihoods, which we commonly denote as $-2 \ln \mathcal{L}_{\text {limits }}$, approximately resembles a $\chi^{2}$ contribution corresponding to one degree of freedom, and can therefore simply be added to the global $\chi^{2}$ function, Eq. (47).

- Exclusion limits from SUSY collider searches:

Lower limits on sfermion and chargino masses from mostly model-independent direct searches at LEP are typically at the level of $\sim 100 \mathrm{GeV}$ (summarized in the PDG review [145]) and are applied in our scan. We furthermore require the lightest supersymmetric particle (LSP) to be the lightest neutralino, however, we do not apply any dark matter relic density constraints.

Exclusions from SUSY searches at Run 1 of the LHC are tested by employing the public computer code CheckMATE-1 . 2 . 2 [146], which includes all relevant $8 \mathrm{TeV}$ SUSY analyses from ATLAS and CMS. However, due to the large computational effort and the large scan samples it is neither feasible nor relevant to test all scan points with CheckMATE. Therefore, in a postprocessing step, we select the most interesting parameter points, i.e. points with a $\chi^{2}$ difference to the minimal $\chi^{2}$ of less than $\sim 10$, and only test the LHC SUSY search constraints on these points. ${ }^{12}$ For each of these

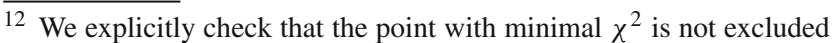
by CheckMATE, or, in case it is excluded, we select more points for
}

points we evaluate the sparticle decay spectrum with SUSY-HIT-1 . 5 [147] and feed these into Herwig ++ (version 2.7.1) [148,149] for Monte-Carlo generation of inclusive sparticle pair production and the evaluation of the leading-order production cross section. It is not computationally feasible to evaluate the NLO corrections to the leading-order cross section for each parameter point. Instead, we multiply the leading-order cross section by an estimated global $k$-factor of 1.5 to approximately account for these corrections. CheckMATE processes the MC events through the implemented ATLAS and CMS analyses and follows a similar statistical procedure as HiggsBounds: It first determines which analysis is the most sensitive one, based on the expected exclusion limit, and then applies the observed exclusion limit from only this search in order to judge whether the parameter point is excluded at the $95 \%$ C.L. or not.

\section{Results}

In this section we discuss the results of our numerical analysis. We first discuss the results for the best fit points in both the light and heavy Higgs case in order to give an impression on the overall fit quality. Then we discuss the preferred parameter space for the light Higgs case in Sect. 4.2. We include a dedicated discussion of the alignment without decoupling scenario for which we select only parameter points with $M_{A} \leq 350 \mathrm{GeV}$. In Sect. 4.3 we present the results for the heavy Higgs case. New benchmark scenarios for the heavy Higgs case for LHC Higgs searches during Run II are presented in Sect. 4.4.

Recall that approximate alignment without decoupling relies on an approximate cancellation between tree-level and loop level contributions to the effective Higgs basis parameter $Z_{6}$ as discussed in Sect. 2.2. The extent of the tuning associated with the regions in the pMSSM 8 scan that exhibit approximate Higgs alignment without decoupling is discussed in Appendix A.

\subsection{Best-fit points and fit quality}

The minimal $\chi^{2}$ over the number of degrees of freedom, $v$, indicates the best achievable level of agreement with the observations within a specific model and defines our best-fit (BF) points. These are summarized in Table 3 for the SM and the two MSSM fits that are separately performed for the light Higgs interpretation $(h)$ and heavy Higgs interpretation $(H)$. The results are given for fits to three different selections of observables (cf. Sect. 3.2): (i) only Higgs data (i.e. Higgs

the CheckMATE test in order to retain the maximal $\chi^{2}$ difference of $\sim 10$ to the minimum. 
Table 3 Global $\chi^{2}$ results with $v$ degrees of freedom from the fits of the SM and the MSSM with either $h$ or $H$ as the LHC signal, the reduced $\chi_{v}^{2} \equiv \chi^{2} / \nu$, and the corresponding $p$ values. The number of degrees of freedom, $v$, are estimated by subtracting the number of free model parameters from the number of observables

Table 4 Pull table for the best-fit (BF) points of the two MSSM Higgs interpretations

\begin{tabular}{|c|c|c|c|c|c|c|c|c|c|}
\hline \multirow[t]{2}{*}{ Case } & \multicolumn{3}{|l|}{ Full fit } & \multicolumn{3}{|c|}{ Fit without $a_{\mu}$} & \multicolumn{3}{|c|}{ Fit without all LEOs } \\
\hline & $\chi^{2} / v$ & $\chi_{v}^{2}$ & $p$ & $\chi^{2} / v$ & $\chi_{v}^{2}$ & $p$ & $\chi^{2} / v$ & $\chi_{v}^{2}$ & $p$ \\
\hline SM & $83.7 / 91$ & 0.92 & 0.69 & $72.4 / 90$ & 0.80 & 0.91 & $70.2 / 86$ & 0.82 & 0.89 \\
\hline$h$ & $68.5 / 84$ & 0.82 & 0.89 & $68.2 / 83$ & 0.82 & 0.88 & $67.9 / 79$ & 0.86 & 0.81 \\
\hline$H$ & $73.7 / 85$ & 0.87 & 0.80 & $71.9 / 84$ & 0.86 & 0.82 & $70.0 / 80$ & 0.88 & 0.78 \\
\hline
\end{tabular}

\begin{tabular}{|c|c|c|c|c|}
\hline \multirow[t]{2}{*}{ Observable } & \multicolumn{2}{|c|}{ Light Higgs case } & \multicolumn{2}{|c|}{ Heavy Higgs case } \\
\hline & Prediction & Pull & Prediction & Pull \\
\hline$M_{h / H}(\mathrm{GeV})$ & 125.20 & +0.034 & 124.15 & -0.29 \\
\hline $\mathrm{BR}\left(B \rightarrow X_{s} \gamma\right)$ & $3.55 \times 10^{-4}$ & +0.185 & $4.17 \times 10^{-4}$ & +1.138 \\
\hline $\mathrm{BR}\left(B_{s} \rightarrow \mu^{+} \mu^{-}\right)$ & $3.03 \times 10^{-9}$ & +0.247 & $3.48 \times 10^{-9}$ & +0.731 \\
\hline $\mathrm{BR}\left(B^{+} \rightarrow \tau^{+} \nu\right)$ & $7.53 \times 10^{-5}$ & -0.424 & $7.38 \times 10^{-5}$ & -0.465 \\
\hline$\delta a_{\mu}$ & $28.8 \times 10^{-10}$ & -0.151 & $27.6 \times 10^{-10}$ & -0.289 \\
\hline$M_{W}(\mathrm{GeV})$ & 80.383 & -0.080 & 80.373 & -0.480 \\
\hline
\end{tabular}

mass and rate measurements as well as the Higgs exclusion likelihoods, right column), (ii) all observables except $a_{\mu}$ (middle column), and (iii) all observables (left column). We furthermore provide the reduced $\chi^{2}$ value, $\chi_{v}^{2} \equiv \chi^{2} / \nu$, as well as the corresponding $p$ value (assuming an idealized $\chi^{2}$ probability distribution) for each scenario in Table 3 .

In total, we have 92 (93) observables in the SM and light Higgs MSSM interpretation (heavy Higgs MSSM interpretation) contributing to the global $\chi^{2}$ value (cf. Sect. 3.2): 85 Higgs signal rate measurements, one Higgs mass measurement, and one (two) Higgs exclusion observable(s) for the SM and light Higgs MSSM interpretation (heavy Higgs MSSM interpretation - here also the LEP exclusion bounds apply), as well as five low energy observables.

We treat the SM as a one-parameter model, where the free parameter is the Higgs mass, $M_{H}$. Its best-fit value is mainly set by the Higgs mass measurement. The $\chi^{2}$ contribution from the Higgs mass measurement is therefore negligible in the SM. In both MSSM cases we have eight free model parameters.

Taking into account only the Higgs data, the minimal $\chi^{2}$ values found in all three cases are very similar, with the lowest value being found in the light Higgs case of the MSSM. However, accounting for the additional degrees of freedom in the two MSSM cases, the overall fit quality is slightly better in the SM. Nevertheless, all three scenarios give very high $p$ values, indicating excellent agreement with the observations in Higgs searches in each case. ${ }^{13}$

\footnotetext{
$\overline{13}$ It should be noted that the Higgs signal rates of certain search channels are based on the same physical degrees of freedom of the model
}

The picture does not change much when the three flavor observables $\mathrm{BR}\left(B \rightarrow X_{s} \gamma\right), \operatorname{BR}\left(B_{s} \rightarrow \mu^{+} \mu^{-}\right)$and $\operatorname{BR}\left(B_{u} \rightarrow \tau \nu_{\tau}\right)$, as well as the $W$ boson mass are included in the fit. In the SM the largest $\chi^{2}$ contributions from these additional observables come from the $W$ boson mass $\left(\chi^{2} \sim 1.5\right)$ and $\operatorname{BR}\left(B_{S} \rightarrow \mu^{+} \mu^{-}\right)\left(\chi^{2} \sim 0.6\right)$. Both MSSM best-fit points yield slightly better agreement with these observables in comparison to the SM.

Taking into account also the anomalous magnetic moment of the muon, $a_{\mu}$, as observable in the fit, the SM receives a large $\chi^{2}$ penalty $\left(\chi^{2} \sim 11.3\right)$ and thus becomes disfavored with respect to the two MSSM interpretations. Both the light and heavy Higgs case of the MSSM are capable to accommodate the $a_{\mu}$ measurement, receiving $\chi^{2}$ contributions of only $\sim 0.3$ and $\sim 1.8$ from this observable at the best fit point, respectively. The various Higgs mass and LEO predictions and the respective pull, defined as $\left(\hat{O}_{i}-O_{i}\right) / \sigma_{i}$, for the $\mathrm{BF}$ points of the two MSSM interpretations are summarized in Table 4.

The parameters for the best-fit points in the light and heavy Higgs case are shown in Table 5. Naturally, the $M_{A}$ values differ significantly, with a value in the decoupling regime for the light Higgs case and a low value $\sim 170 \mathrm{GeV}$ in the heavy Higgs case. Large mixing in the stop sector is required

and thus their predictions cannot be varied independently. For example, in the MSSM, the predicted rates in $h \rightarrow Z Z^{*}$ and $h \rightarrow W W^{*}$ searches are directly related. The effective number of degrees of freedom is thus lower, see e.g. Ref. [150] for a detailed discussion and analysis. However, since the naive $p$ value found in the study presented here is of $\mathcal{O}(>50 \%)$, no significant change of the conclusion on the validity of the model can be expected from a pseudo-data based study as performed in Ref. [150]. 
Table 5 MSSM parameters for the BF points found for the light Higgs $(h)$ and heavy Higgs $(H)$ interpretation in the full fit

\begin{tabular}{lllllllll}
\hline Case & $M_{A}(\mathrm{GeV})$ & $\tan \beta$ & $\mu(\mathrm{GeV})$ & $A_{t}(\mathrm{GeV})$ & $M_{\tilde{q}_{3}}(\mathrm{GeV})$ & $M_{\tilde{\ell}_{3}}(\mathrm{GeV})$ & $M_{\tilde{\ell}_{1,2}}(\mathrm{GeV})$ & $M_{2}(\mathrm{GeV})$ \\
\hline$h$ & 929 & 21.0 & 7155 & 4138 & 2957 & 698 & 436 & 358 \\
$H$ & 172 & 6.6 & 4503 & -71 & 564 & 953 & 262 & 293 \\
\hline
\end{tabular}

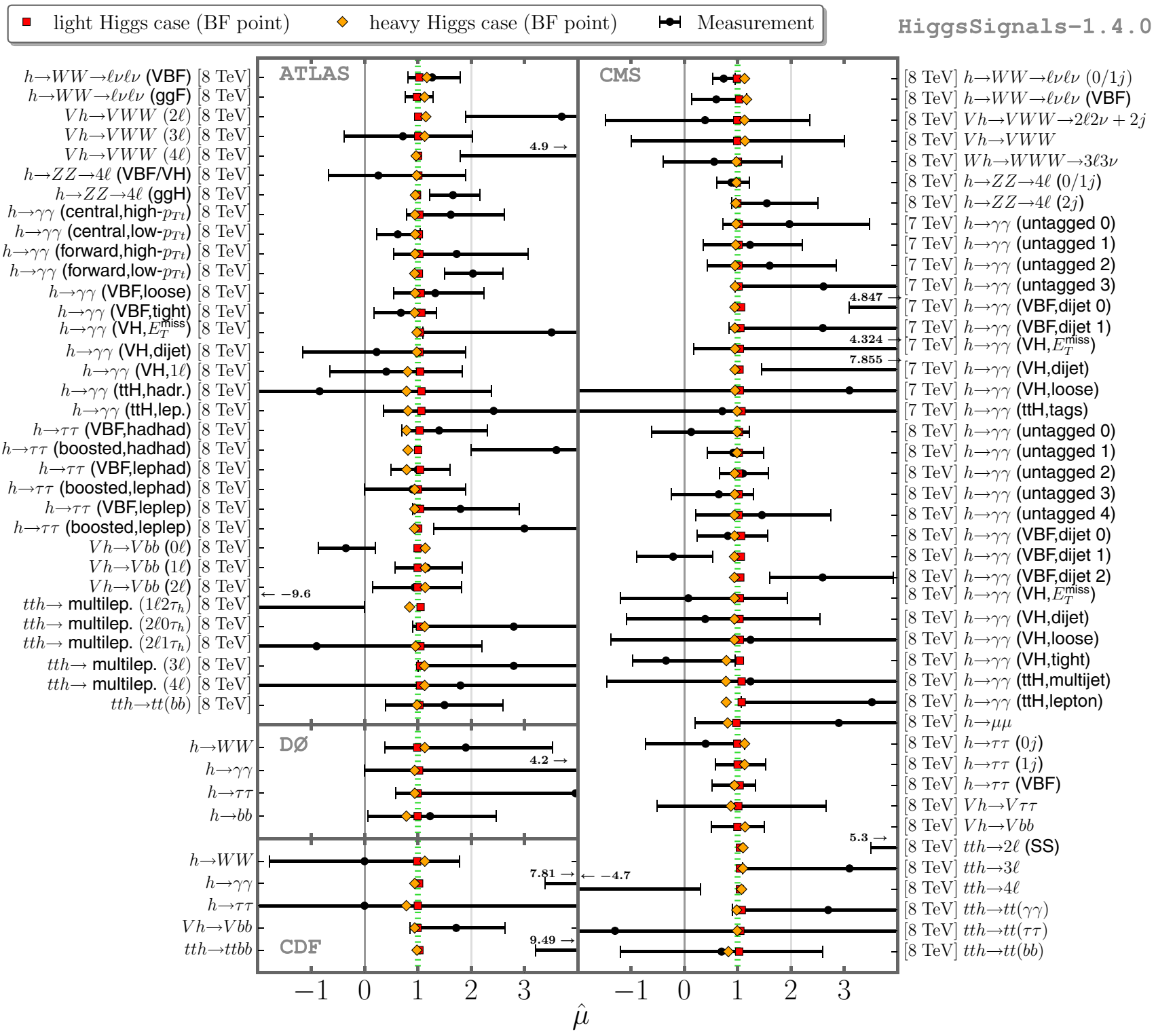

Fig. 3 Comparison of Higgs signal rates in terms of signal strength modifiers $\mu$ between the BF point predictions for the MSSM light Higgs case (red squares) and heavy Higgs case (orange diamonds) and the measurements from the LHC and Tevatron experiments (black dots with error bars). Displayed are all 85 Higgs rate observables that are provided by HiggsSignals-1.4.0 and included in our fit in the light Higgs case to yield $M_{h} \sim 125 \mathrm{GeV}$. As explained above, large higher-order corrections are also required in the heavy Higgs case. For the best fit value in the latter case the trilinear coupling $A_{t}$ is small, while $X_{t}$ is still sizable because of the large contribution from the term $\mu / \tan \beta$. We find large and positive values of $\mu$ for the best fit points in both scenarios. In the light Higgs case we find parameter points providing a very good fit in the entire positive $\mu$ range (as we will discuss below), whereas in the heavy Higgs case large values for $\mu / M_{S}$ are crucial to achieve the approximate 
alignment limit. The scalar leptons of the first and second generation are relatively light to accommodate $a_{\mu}$, and also the preferred value for $M_{2}$ is relatively low.

A comparison of the Higgs signal rates - given in terms of signal strength modifiers $\mu$, which are defined by the measured value of $\sigma \times \mathrm{BR}$ in the respective channel normalized to the SM prediction (see e.g. Ref. [95]) - between the predictions of the BF points in the light and heavy Higgs case and the Tevatron and LHC measurements is displayed in Fig. 3. The BF point predictions are closely centered around the SM prediction $(\mu=1)$, especially in the light Higgs case, where deviations from the SM prediction are $\lesssim \mathcal{O}(2-3 \%)$. For the heavy Higgs case BF point the deviations are slightly larger $\lesssim \mathcal{O}(10 \%)$, however, these mostly appear in less accurately measured channels.

\subsection{The light Higgs interpretation}

We start our presentation of the fit results in the light Higgs interpretations of the MSSM with the predicted Higgs signal rates and their correlations for the preferred parameter points. We then give an overview of the preferred MSSM parameter regions. Here we separate the discussion between the full parameter space and a region with low $\mathcal{C} \mathcal{P}$-odd Higgs mass, $M_{A} \leq 350 \mathrm{GeV}$. The latter selects most of the preferred parameter points that feature the limit of alignment without decoupling. The last two subsections provide dedicated discussions of the impact of the low energy observables and direct LHC SUSY searches on the fit.

\subsubsection{Higgs signal rates}

In Fig. 4 we show the $\Delta \chi_{h}^{2}=\chi_{h}^{2}-\chi_{h \text {, min }}^{2}$ distributions (the subscript ' $h$ ' refers to the light Higgs ( $h$ ) interpretation), based on all observables, for four different Higgs signal rates, defined by

$R_{X X}^{P(h)}=\frac{\sum_{P(h)} \sigma(P(h)) \times \mathrm{BR}(h \rightarrow X X)}{\sum_{P(h)} \sigma_{\mathrm{SM}}(P(h)) \times \mathrm{BR}_{\mathrm{SM}}(h \rightarrow X X)}$.

Here $X X=V V, \gamma \gamma, b b, \tau \tau$ (with $V=W^{ \pm}, Z$ ) denotes the final state from the Higgs decay and $P(h)$ denotes the Higgs production mode. For inclusive Higgs production, $P(h) \equiv h$, the sum in Eq. (48) runs over the five dominant Higgs production modes: gluon-gluon fusion (ggf), vector boson fusion (VBF), associated Higgs production with a $W$ or $Z$ boson, ( $V H$, with $V=W^{ \pm}, Z$ ) and Higgs production in association with a top quark pair $(t \bar{t} H)$. The subscript 'SM' denotes the quantities as predicted in the SM, whereas no subscript refers to the quantity predicted in the model. Parameter points that pass (do not pass) the constraints from Higgs exclusion limits, tested via HiggsBounds, and direct SUSY LHC searches, tested via CheckMATE, are given as blue (gray) points.

The preferred Higgs signal rates are

$R_{V V}^{h}=0.99_{-0.08}^{+0.09}, \quad R_{\gamma \gamma}^{h}=1.02_{-0.10}^{+0.16}$,

$R_{b b}^{V h}=1.00_{-0.05}^{+0.02}, \quad R_{\tau \tau}^{h}=1.00_{-0.20}^{+0.06}$,

where the upper and lower values are determined by $\Delta \chi_{h}^{2} \leq$ 1 , which approximately corresponds to the one-dimensional $68 \%$ C.L. interval. All central values lie very close to the SM prediction $(R=1)$.

The narrowest range is found for the Higgs signal rate $R_{b b}^{V h}$, i.e. for $V h$ production with the Higgs boson decaying into $b \bar{b}$. This is mainly because a direct variation of $\mathrm{BR}(h \rightarrow b \bar{b})$ through a modification of the partial width $\Gamma(h \rightarrow b \bar{b})$ also leads to a substantial change of the total Higgs decay width, $\Gamma_{h \text {,tot }}$, and thus affects significantly the branching ratios of all other Higgs decay modes. This global rescaling of the branching ratios of all decay modes except $h \rightarrow b \bar{b}$ could only be compensated by an inverse rescaling in the rates of all Higgs production modes. However, this cannot be accomplished within the MSSM, and hence the modification of $\mathrm{BR}(h \rightarrow b \bar{b})$ is severely constrained.

In Fig. 4 we can furthermore observe a spread of parameter points deviating substantially from the SM value, $R=1$, within the $\Delta \chi^{2} \leq 4$ interval (approximately corresponding to the $95 \%$ C.L. interval), e.g. $R_{\gamma \gamma}^{h} \lesssim 1.35$ and $R_{\tau \tau}^{h} \gtrsim 0.65$. Such modifications can easily appear in the MSSM: An enhancement of the $h \rightarrow \gamma \gamma$ partial decay width can appear through the loop contribution of light charged SUSY particles such as light scalar tau leptons (staus) or charginos. ${ }^{14}$ The observed reduction of $R_{\tau \tau}^{h}$ for some points in Fig. 4 originates from a simultaneous (but small) suppression of the gluon fusion production cross section, $\sigma(g g \rightarrow h)$, and the decay rate $\mathrm{BR}\left(h \rightarrow \tau^{+} \tau^{-}\right)$with respect to their SM predictions.

The two-dimensional correlations among the Higgs signal rates are shown in Fig. 5. Compared to Fig. 4 we introduced two new colors in order to indicate regions close to the minimum $\chi^{2}$. Points with $\Delta \chi_{h}^{2}<2.30$ (5.99) are highlighted in red (yellow), corresponding to points in a two-dimensional $68 \%(95 \%)$ C.L. region in the Gaussian limit. We shall denote these regions simply by favored/preferred (yellow) and most favored/preferred regions (red). The best fit point is indicated by a black star in the figures.

As already noticed in our previous analysis [19] the diphoton rate $R_{\gamma \gamma}^{h}$ exhibits a strong correlation with $R_{V V}^{h}$ and a strong anti-correlation with $R_{b b}^{V h}$. The latter arises through

\footnotetext{
${ }^{14}$ In the analysis of Ref. [19], based on the data available at that time, contributions of this kind leading to a substantial increase in $R_{\gamma \gamma}^{h}$ were favored, whereas with the new data this enhancement turns out to be much smaller.
} 

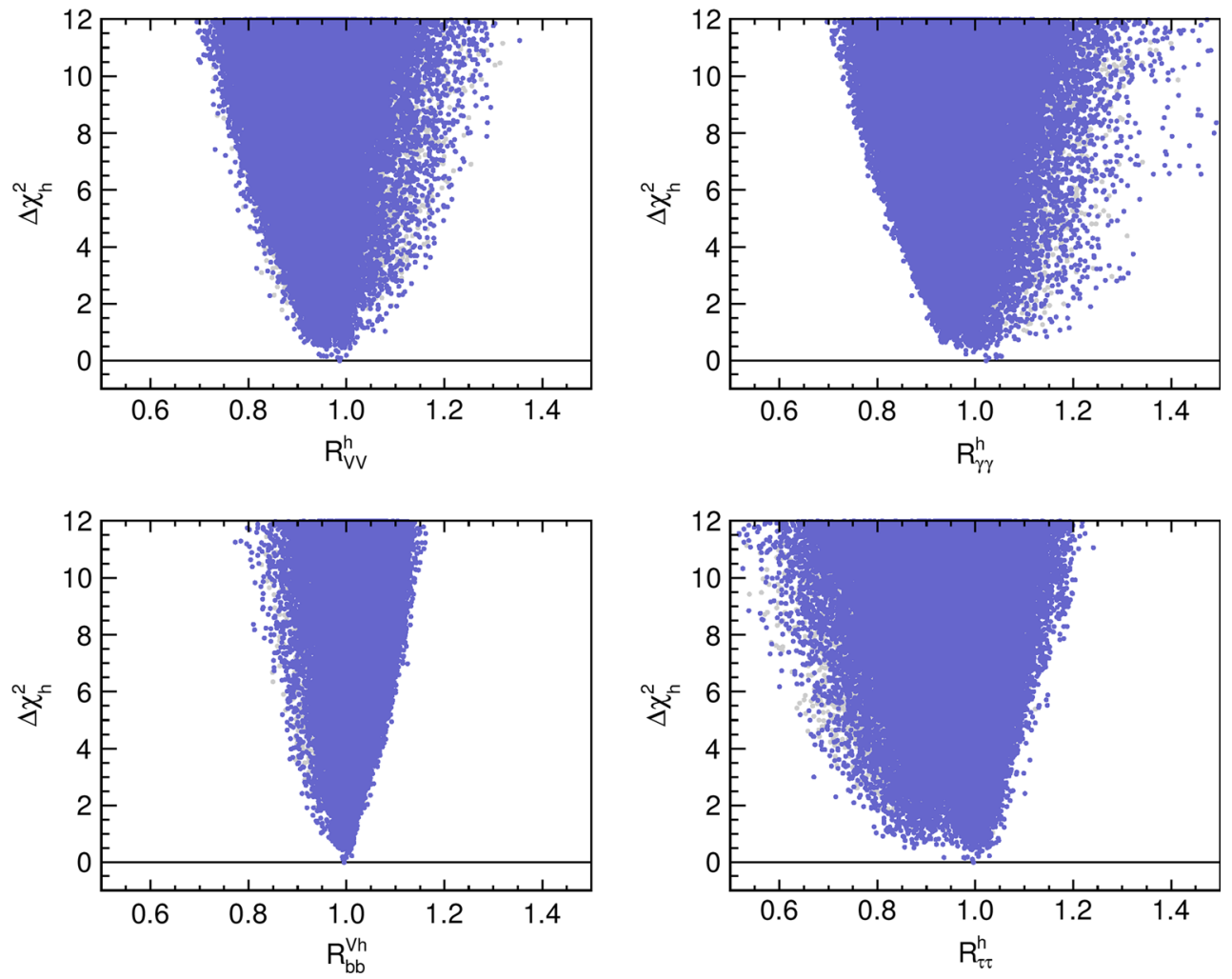

Fig. $4 \Delta \chi_{h}^{2}=\chi_{h}^{2}-\chi_{h \text {, } m i n}^{2}$ distributions in the four dominant Higgs signal rates (defined in the text) for the light Higgs interpretation. Points that pass (do not pass) the direct constraints from Higgs searches

from HiggsBounds and from LHC SUSY particle searches from CheckMATE are shown in blue (gray)
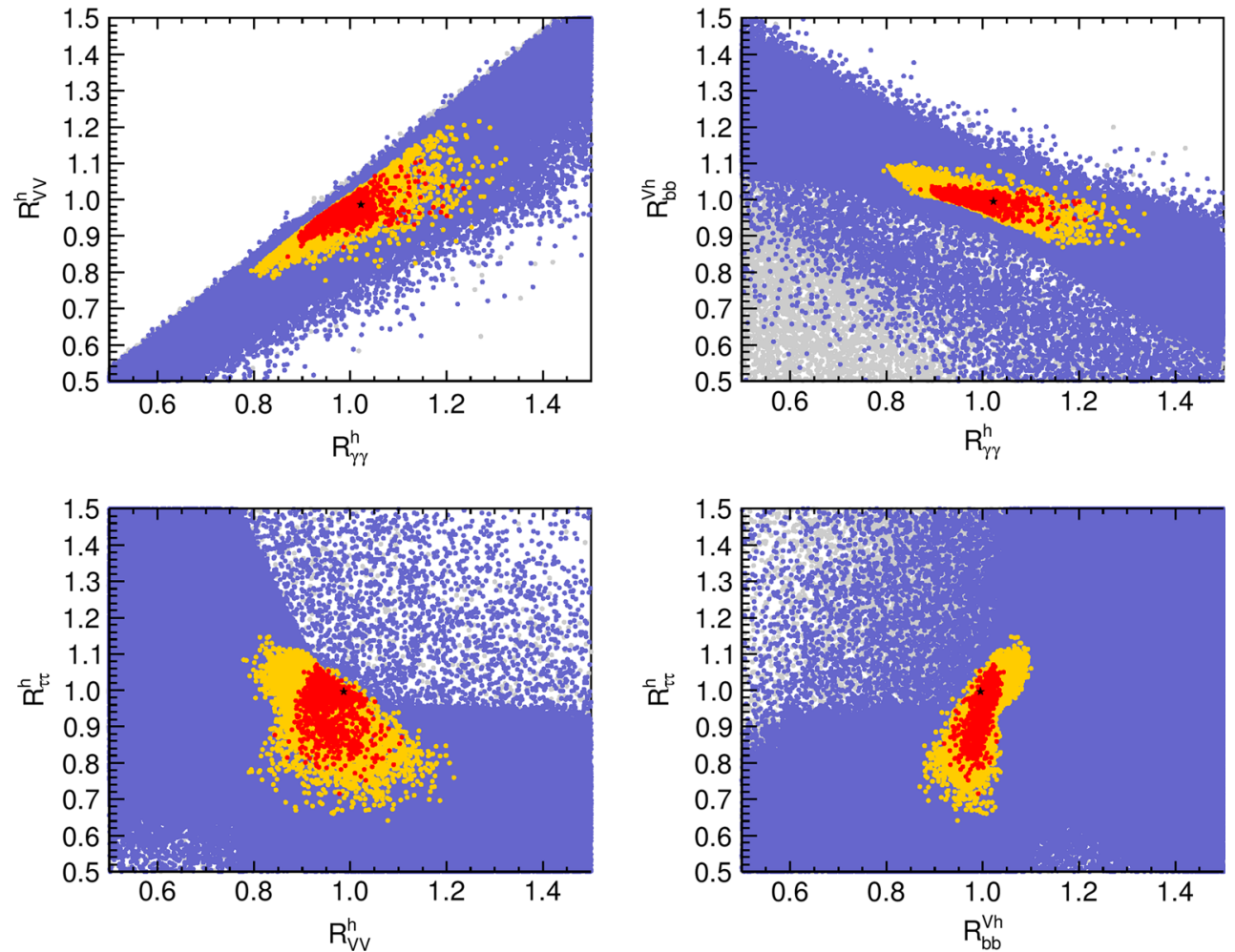

Fig. 5 Correlations between Higgs signal rates for the light Higgs case. The color coding follows that of Fig. 4, with the addition of the favored regions with $\Delta \chi_{h}^{2}<2.3$ (red) and $\Delta \chi_{h}^{2}<5.99$ (yellow). The best fit point is indicated by a black star 

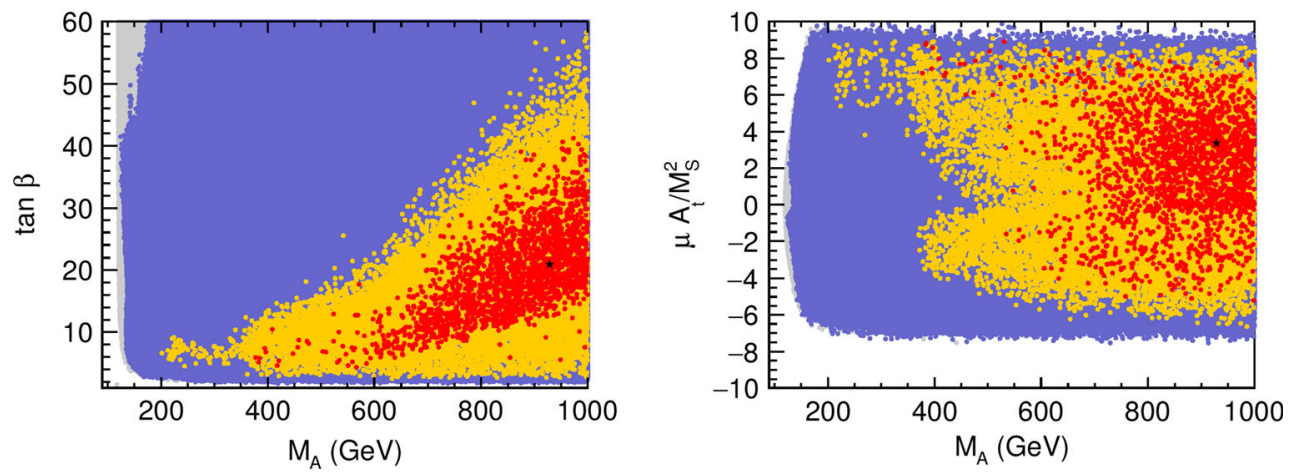

Fig. 6 Preferred parameter regions in the $\left(M_{A}, \tan \beta\right)$ plane (left) and the $\left(M_{A}, \mu A_{t} / M_{S}^{2}\right)$ plane (right) for the light Higgs case. The color coding is the same as in Fig. 5

the strong influence of the $h \rightarrow b \bar{b}$ partial width on the total Higgs decay width (see the discussion above). In contrast, the rate $R_{\tau \tau}^{h}$ only shows mild correlations with $R_{V V}^{h}, R_{\gamma \gamma}^{h}$ (not shown here) and $R_{b b}^{V h}$. The latter is easily understood from the fact that the same Higgs doublet couples to down-type quarks and leptons in the MSSM, thus, at tree-level, the light Higgs coupling to $\tau$ leptons and $b$ quarks is affected in the same way (and enhanced at large $\tan \beta$ ). However, we also find favoured points that feature a significant suppression of $R_{\tau \tau}^{h}$ while $R_{b b}^{h}$ shows no (or only a small) suppression. Differences between $R_{\tau \tau}^{h}$ and $R_{b b}^{h}$ arise from loop contributions, which modify the $h \tau^{+} \tau^{-}$and $h b \bar{b}$ couplings in a different manner, see e.g the discussion of $\Delta_{b}$ corrections in Ref. [19].

\subsubsection{Parameter space}

The distribution of preferred parameter points in the plane of the parameters $M_{A}$ and $\tan \beta$, which determine the Higgs sector at lowest order, is shown in Fig. 6 (left). The bulk of the favored points is found at large $\mathcal{C P}$-odd Higgs mass values, $M_{A} \gtrsim 350 \mathrm{GeV}$, i.e. in a region where the decoupling limit is already approximately realized. Large $\tan \beta$ values at moderate values of $M_{A}$ are disfavored by the non-observation of a signal in LHC $H / A \rightarrow \tau^{+} \tau^{-}$searches, which is incorporated in our study by adding the exclusion likelihood from CMS (provided by HiggsBounds) to the global $\chi^{2}$ function instead of applying the hard cut at the $95 \%$ C.L. (see Sect. 3.3) [23]. Thus, points excluded (e.g. at the 95\% C.L.) by the CMS search alone can still appear as blue points here, but are unlikely to show up as yellow or red points.

While all most favored (red) points are found for $M_{A} \gtrsim$ $350 \mathrm{GeV}$, some preferred parameter points (yellow) are found at low $\mathcal{C P}$-odd Higgs masses down to $M_{A} \gtrsim 170 \mathrm{GeV}$ in a narrow range of $\tan \beta$ values $\sim 4-10$. These points are far away from the decoupling limit, however, they turn out to feature an (approximate) realization of the limit of alignment without decoupling. As discussed in Sect. 2.2 these points must have either large values of $\mu A_{t} / M_{S}^{2}$ or, in the case where $\left|A_{t} / M_{S}\right|$ is small, even larger values of $\left|\mu / M_{S}\right|$ (beyond $\sim 3$ ) in order to achieve the Higgs alignment limit at reasonably small values of $\tan \beta$ which are unexcluded by LHC $H / A \rightarrow \tau^{+} \tau^{-}$searches. This is indeed the case, as can be seen in Fig. 6 (right), where we show the preferred parameter points in the $\left(M_{A}, \mu A_{t} / M_{S}^{2}\right)$ plane. All preferred parameter points with $M_{A} \lesssim 350 \mathrm{GeV}$ have $\mu A_{t} / M_{S}^{2}$ values of at least 3 , but typically between 5 and 9 . It should be noted that in the light Higgs scan we constrain both scan parameters $\mu$ and $A_{t}$ to be $\leq 3 M_{\tilde{q}_{3}}$ (see Table 1), limiting the possible values of $\mu A_{t} / M_{S}^{2}$ to $\lesssim 9$. In the following, in order to study the parameter points close to the limit of alignment without decoupling, we apply a cut $M_{A} \leq 350 \mathrm{GeV}$ (denoted as low$M_{A}$ selection) whenever relevant to isolate these points from the other parameter points.

The MSSM parameters from the stop sector, namely the stop mixing parameter, $X_{t} / M_{S}$, and the light stop mass, $m_{\tilde{t}_{1}}$, are shown in Fig. 7 for the full scan (left) and the low- $M_{A}$ selection (right). In the full scan we find preferred parameter points in both the positive and negative $X_{t} / M_{S}$ branches near the value where the contribution to the Higgs mass from stop mixing is maximized, $\left|X_{t} / M_{S}\right| \sim 2 .{ }^{15}$ Light stop masses, $m_{\tilde{t}_{1}}$, down to values $\gtrsim 300$ (400) $\mathrm{GeV}$ are possible in the positive (negative) $X_{t} / M_{S}$ branch. ${ }^{16}$ In the low- $M_{A}$ selection preferred parameter points are found only in the positive $X_{t} / M_{S}$ branch for $X_{t} / M_{S} \gtrsim 2$. Here, the lowest light stop mass value in the preferred parameter region is found at around $m_{\tilde{t}_{1}} \sim 580 \mathrm{GeV}$.

In order to understand why the favored parameter points near the limit of alignment without decoupling are found only at $X_{t} / M_{S}=A_{t} / M_{S}-\left(\mu / M_{S}\right) \cot \beta \gtrsim 2$, we show the cor-

\footnotetext{
15 The highest $M_{h}$ values are reached for $\left|X_{t} / M_{S}\right| \sim 2$ due to the inclusion of the higher-order corrections in the on-shell renormalization scheme, see Ref. [151] for details.

16 Note that we assumed universality of the left- and right-handed softbreaking stop mass parameter here. Lower light stop masses even below the top quark mass can be obtained while being consistent with the Higgs rates in the presence of a large mass splitting in the stop sector [152].
} 

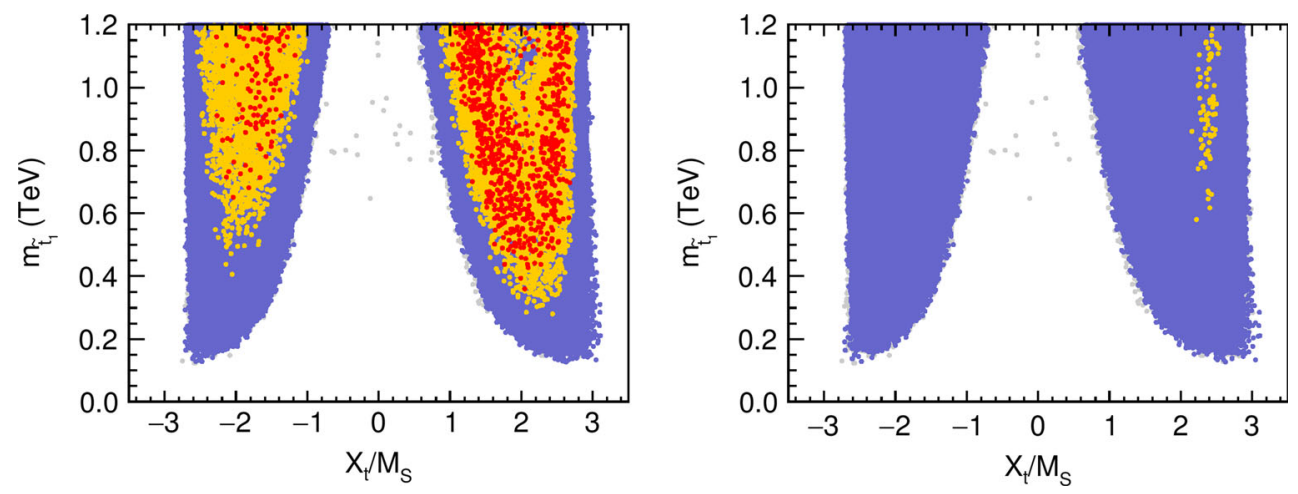

Fig. 7 Stop mixing parameter, $X_{t} / M_{S}$, versus the light stop mass, $m_{\tilde{t}_{1}}$, for the light Higgs case for the full scan $(l e f t)$ and the low- $M_{A}$ selection (right). The color coding is the same as in Fig. 5
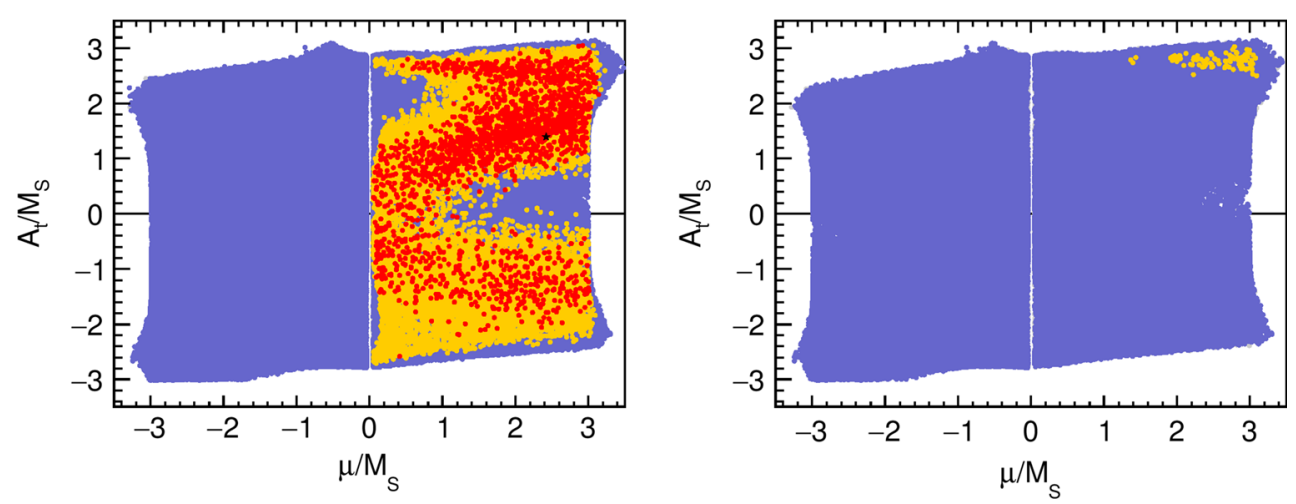

Fig. 8 Preferred parameter regions in the $\left(\mu / M_{S}, A_{t} / M_{S}\right)$ plane for the full fit (left) and the low- $M_{A}$ selection (right). The color coding is the same as in Fig. 5

relations of the parameters $A_{t} / M_{S}$ and $\mu / M_{S}$ in Fig. 8 for the full scan (left) and the low- $M_{A}$ selection (right). While we find preferred parameter points at both positive and negative $A_{t} / M_{S}$ values for $\mu / M_{S}>0$ in the full scan, the low- $M_{A}$ selection features favored points only for very large and positive values of $A_{t} / M_{S}$ and $\mu / M_{S}$. In particular, we find most of the preferred parameter points in the low- $M_{A}$ region in a narrow range $2.4 \lesssim A_{t} / M_{S} \lesssim 3$, while the range in $\mu / M_{S}$ is larger (roughly between 1.4 and 3 ). In the full scan (left) it can be seen that even values with $A_{t} / M_{S}$ and $\mu / M_{S}$ close to zero can yield a very good fit. Consequently, the quite large best fit value of $\mu$, see also Table 5 , should be regarded as accidental.

In the following we will analyze in detail which observables and constraints lead to this particular favored region of parameter space in the alignment limit. We recall the parametric dependence of the approximate one-loop alignment condition in the limit $\left|\mu A_{t}\right| \tan \beta \gg M_{S}^{2}$, (21),

$\tan \beta \sim\left(\frac{\mu A_{t}}{M_{S}^{2}}\left[\frac{A_{t}^{2}}{M_{S}^{2}}-6\right]\right)^{-1}$
In order to find viable solutions of this condition (while restricting ourselves to $\left|A_{t}\right| / M_{S} \leq 3$ and $|\mu| / M_{S} \leq 3$ ) we need to have ${ }^{17}$

$A_{t} \begin{cases}>\sqrt{6} M_{S} & \text { if } \mu A_{t}>0 \\ <\sqrt{6} M_{S} & \text { if } \mu A_{t}<0\end{cases}$

We illustrate the impact of the various constraints and observables on the possible solutions for the limit of alignment without decoupling in the $\left(\mu / M_{S}, A_{t} / M_{S}\right)$ plane in Fig. 9. Here we plot only the parameter points in the low- $M_{A}$ selection with $\Delta \chi^{2} \leq 5.99$ (approximately corresponding to the $95 \%$ C.L. region) based on different sets of observables: only Higgs mass and signal rates (top left), Higgs mass, signal rates and $h / H / A \rightarrow \tau^{+} \tau^{-}$exclusion likelihood (upper right), all observables except $a_{\mu}$ (lower left) and all observables (lower right). In color we indicate the $\tan \beta$ value of the parameter points (see legend). The upper left plot of Fig. 9 shows all possible regions where the alignment condition, $Z_{6} v^{2}=0$, (cf. (17)), is approximately fulfilled.

17 Note that higher-order corrections as discussed in Sect. 2.2 can modify the numbers given here. 

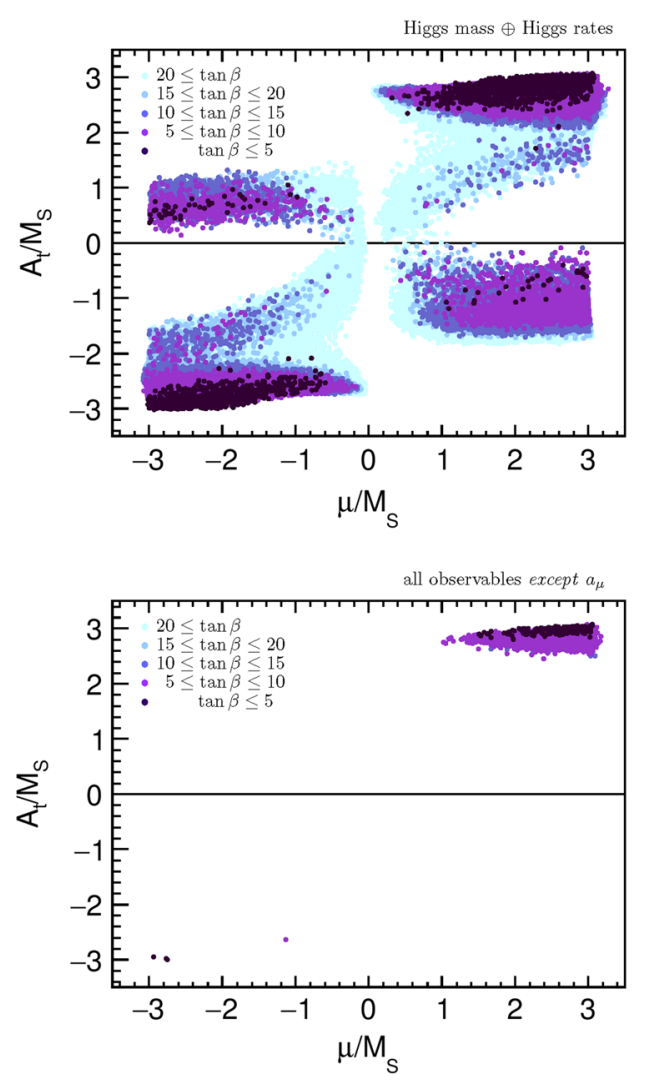

Fig. $9 \mu / M_{S}$ vs. $A_{t} / M_{S}$ for the preferred points with low $\mathcal{C} \mathcal{P}$-odd Higgs mass, $M_{A} \leq 350 \mathrm{GeV}$, for different selection of observables. The color indicates the $\tan \beta$ value of the parameter points (see legend). The points are within the (approximate) 95\% C.L. region, based

These are found in each quadrant labeled by the algebraic signs of $\left(\mu / M_{S}, A_{t} / M_{S}\right):(+,+),(+,-),(-,+),(-,-)$ (cf. also the discussion of Fig. 1 in Sect. 2.2). It can clearly be seen that $\left|A_{t} / M_{S}\right|<\sqrt{6}$ is required in the $(+,-)$ and $(-,+)$ quadrants, consistent with $(51)$, and that these parameter points tend to have larger $\tan \beta$ values than those in the $(+,+)$ and $(-,-)$ quadrants. Once the constraints from LHC $H / A \rightarrow \tau^{+} \tau^{-}$searches are taken into account, as shown in the upper right plot of Fig. 9, the large $\tan \beta$ points and thus the $(+,-)$ and $(-,+)$ quadrants - become strongly disfavored. Small $\left|A_{t}\right|$ values in the $(+,+)$ and $(-,-)$ quadrants also require larger $\tan \beta$ and are thus equally disfavored. Adding also the flavor observables $\operatorname{BR}\left(B \rightarrow X_{s} \gamma\right)$, $\mathrm{BR}\left(B_{s} \rightarrow \mu^{+} \mu^{-}\right)$and $\mathrm{BR}\left(B^{+} \rightarrow \tau^{+} \nu_{\tau}\right)$ as well as the $W$ boson mass observable to the fit, the negative $\mu$ region (as well as the regions with $\mu A_{t}<0$ ) become mostly disfavored, as shown in the lower left plot of Fig. 9. Interestingly, this feature emerges already without including the observable $a_{\mu}$ in the fit. The negative $\mu$ region is mostly disfavored by $\operatorname{BR}\left(B \rightarrow X_{s} \gamma\right)$, while the negative $\mu A_{t}$ region is disfavored by $\operatorname{BR}\left(B_{s} \rightarrow \mu^{+} \mu^{-}\right)$, as we will discuss in more detail in the next section. In addition, the negative $\mu$ region becomes strongly disfavored after adding $a_{\mu}$ to the fit, as the
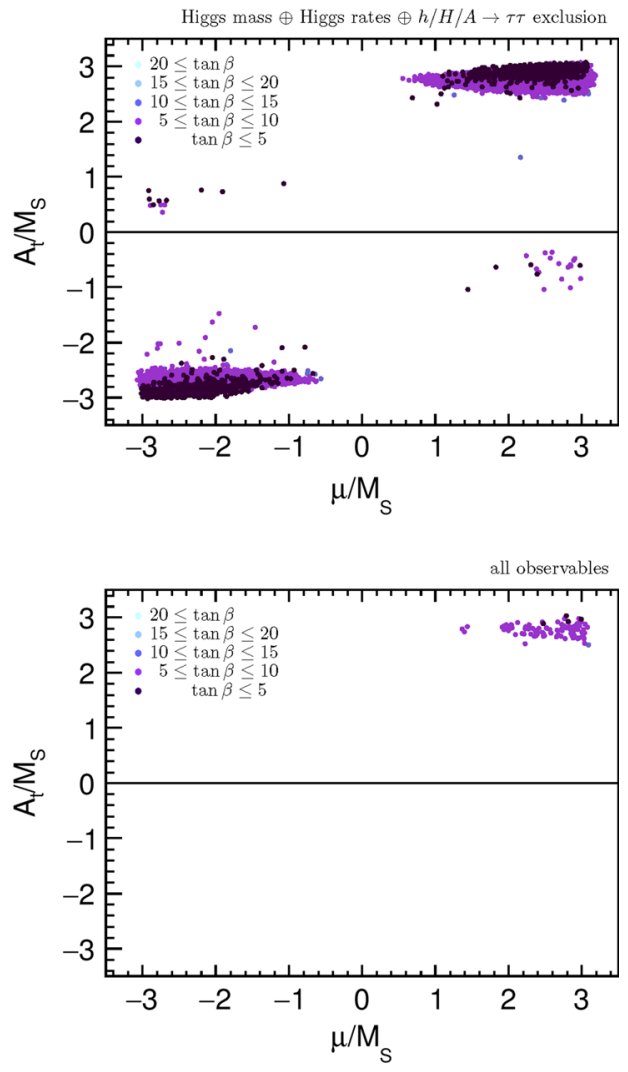

on the following selection of observables: only Higgs mass and signal rates (upper left), Higgs mass, signal rates and $h / H / A \rightarrow \tau^{+} \tau^{-}$exclusion likelihood (upper right), all observables except $a_{\mu}$ (lower left), all observables (lower right)

sign of the SUSY contribution to $a_{\mu}$ depends on the sign of $\mu$. Thus $\mu$ needs to be positive in order to account for the currently observed discrepancy between measurement and theory prediction. This is shown in the lower right plot of Fig. 9 where only points with positive $\mu$ and $A_{t}$ at low $\tan \beta$ values remain, reproducing the distribution of favored points in the right plot of Fig. 8.

\subsubsection{Impact of low energy observables}

In this section we discuss the interplay of the Higgs observables and limits from Higgs searches with the low-energy observables, in particular the rare $B$ decays, in the global fit. The light Higgs case features a very good fit to all lowenergy observables as we have already seen in Tables 3 and 4. For the most part we concentrate on the low- $M_{A}$ selection and study the low-energy observables for the parameter points close to the limit of alignment without decoupling. This is particularly interesting since the low $M_{A}$ value implies that all MSSM Higgs bosons, and in particular the charged Higgs boson, are relatively light, which can lead to large contributions to the $B$ decays. In contrast, the MSSM (loop-)contributions to the anomalous magnetic moment of 


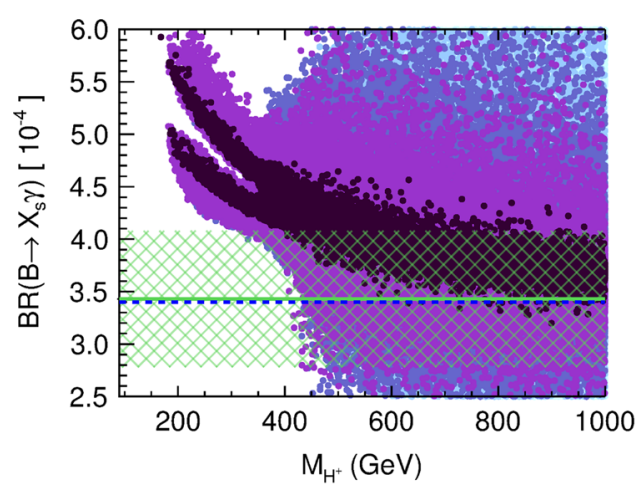

Fig. $10 \operatorname{BR}\left(B \rightarrow X_{s} \gamma\right)$ vs. $M_{H^{ \pm}}$(left) and $\mu / M_{S}$ (right) for the favored points in the fit without taking into account the LEOs. In the right plot only points with $M_{A}<350 \mathrm{GeV}$ are shown. The green line and hatched region indicate the corresponding experimental measure-

the muon and the $W$ boson mass are dominated by light squarks and sleptons, effects from MSSM Higgs bosons are not very pronounced.

The leading contribution to the FCNC process $b \rightarrow s \gamma$ occurs in the SM via a $W^{ \pm}-t$ loop, allowing new-physics contributions to be of similar size. The branching ratio $\mathrm{BR}\left(B \rightarrow X_{s} \gamma\right)$ can receive sizable positive contributions from an $H^{ \pm}-t$ loop if the charged Higgs boson mass is not too large. It was recently pointed out [153] that $b \rightarrow s \gamma$ excludes charged Higgs bosons with $M_{H^{ \pm}}<480 \mathrm{GeV}$ at the $95 \%$ C.L. in a 2HDM with Type-II Yukawa couplings $[53,66,154]$. However, as discussed in Sect. 2.3, wrong-Higgs Yukawa couplings are induced radiatively by SUSY-breaking effects in the MSSM. These lead to important modifications of the charged Higgs couplings to up- and down-type quarks [cf. Eq. (43)], which in turn change the $\operatorname{BR}\left(B \rightarrow X_{s} \gamma\right)$ prediction in the MSSM from the corresponding prediction in the Type-II 2HDM according to Eq. (44). In particular, if $\Delta_{b}$ [given by Eq. (39)] is positive, then the MSSM prediction for $\operatorname{BR}\left(B \rightarrow X_{s} \gamma\right)$ is smaller than in the Type-II 2HDM. Furthermore, supersymmetric particles in the loop can also contribute to the $b \rightarrow s \gamma$ amplitude. For example, charginostop $\left(\tilde{\chi}^{ \pm}-\tilde{t}\right)$ loops can contribute with either sign, depending on sign and magnitude of the parameters $\mu, M_{2}$ and $A_{t}$, and thus may partially cancel the effects of the $H^{ \pm}-t$ loop.

The impact of the LEOs on the global fit is illustrated best by studying the LEO predictions of the parameter points that are preferred before the LEOs are included in the fit. In the following we therefore focus on the (approximate) 95\% C.L. preferred parameter points in the low- $M_{A}$ selection after the Higgs signal rates, Higgs mass and $h / H / A \rightarrow \tau^{+} \tau^{-}$exclusion likelihood are included in the fit, i.e. the points in the upper right plot of Fig. 9. The left plot in Fig. 10 shows the charged Higgs mass dependence of $\operatorname{BR}\left(B \rightarrow X_{s} \gamma\right)$. Generally we observe that for light charged Higgs values corre-

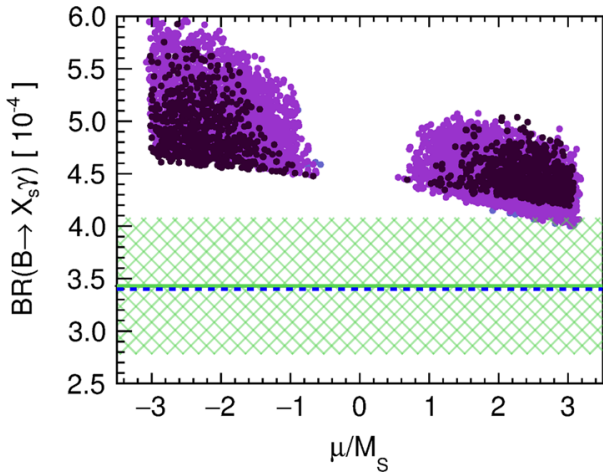

ments and the total $1 \sigma$ uncertainty region, while the SM prediction is indicated by the blue dashed line. The color coding of the displayed points is the same as in Fig. 9

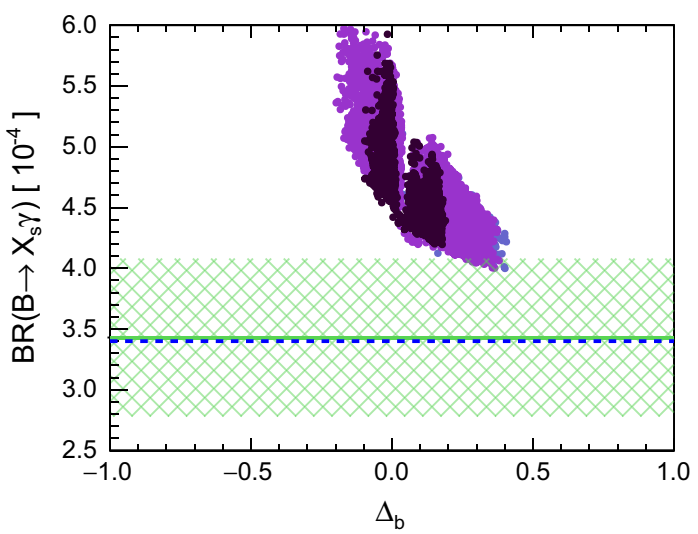

Fig. $11 \operatorname{BR}\left(B \rightarrow X_{s} \gamma\right)$ vs. $\Delta_{b}$ for the favored points in the fit without taking into account the LEOs, for the low $M_{A}$ selection $\left(M_{A}<\right.$ $350 \mathrm{GeV}$ ). The green and blue line and the hatched region are the same as in Fig. 10. The color coding of the displayed points is the same as in Fig. 9

sponding to the low- $M_{A}$ selection (slightly) too large predictions for BR $\left(B \rightarrow X_{s} \gamma\right)$ are obtained. For $M_{H^{ \pm}} \lesssim 350 \mathrm{GeV}$ we do not find any preferred points within the $1 \sigma$ region of the experimental measurement, which is indicated by the green band. One can see that the $\operatorname{BR}\left(B \rightarrow X_{s} \gamma\right)$ prediction tends to increase when going to smaller charged Higgs masses, $M_{H^{ \pm}}$. The two branches in this parameter region visible at low $M_{A}$ values correspond to $\mu>0$ (lower branch) and $\mu<0$ (upper branch), which can also be seen in the right plot in Fig. 10, where $\operatorname{BR}\left(B \rightarrow X_{s} \gamma\right)$ is shown as a function of $\mu / M_{S}$ (for $M_{A}<350 \mathrm{GeV}$ ). The lower branch lies mostly within the $2 \sigma$ region of the experimental measurement whereas most of the upper branch is inconsistent with the measurement at the $2 \sigma$ level. This confirms our previous statement that negative $\mu$ is disfavored by $B \rightarrow X_{s} \gamma$.

The $\mu$ dependence of the $\operatorname{BR}\left(B \rightarrow X_{s} \gamma\right)$ prediction is easily understood from the discussion in Sect. 2.3. In the 


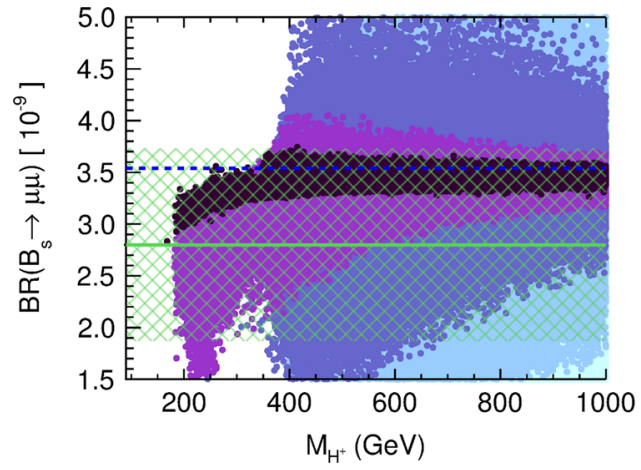

Fig. $12 \operatorname{BR}\left(B_{s} \rightarrow \mu^{+} \mu^{-}\right)$in dependence of the charged Higgs mass, $M_{H^{ \pm}}$, (left) and $\mu A_{t} / M_{S}^{2}$ (right) for the favored points in the fit without taking into account the LEOs. In the right plot only points with $M_{A}<350 \mathrm{GeV}$ are shown. The green line and hatched region indicate

approximation $M_{Z}, M_{A} \ll M_{S}, \Delta_{b}$ is large and positive [negative] for large positive [negative] $\mu$, thereby leading to a substantial decrease [increase] of $\operatorname{BR}\left(B \rightarrow X_{s} \gamma\right)$ with respect to the Type-II $2 \mathrm{HDM}$ prediction [cf. Eq. (44)]. This $\Delta_{b}$ dependence is also directly shown in Fig. 11 for the parameter region with $M_{A} \leq 350 \mathrm{GeV}$. The color coding furthermore illustrates that $\Delta_{b} \propto \tan \beta$, i.e. the largest suppression of $\operatorname{BR}\left(B \rightarrow X_{s} \gamma\right)$ is obtained for large, positive $\mu / M_{S}$ and large $\tan \beta$. Note that the chargino-stop contributions to $\operatorname{BR}\left(B \rightarrow X_{s} \gamma\right)$ are found to be relatively small in this parameter region and thus play only a minor role in this discussion.

The decay $B_{s} \rightarrow \mu^{+} \mu^{-}$can be mediated in the MSSM by a neutral Higgs boson and receives loop corrections involving squarks, sleptons and electroweakinos. As can be seen in Fig. 12, in the limit of alignment without decoupling we find a very good fit to $\operatorname{BR}\left(B_{S} \rightarrow \mu^{+} \mu^{-}\right)$, while for larger values of $M_{H^{ \pm}}$larger deviations are possible. In the right plot of Fig. 12, where $\operatorname{BR}\left(B_{s} \rightarrow \mu^{+} \mu^{-}\right)$is shown as a function of $\mu A_{t} / M_{S}^{2}$ (for $M_{A}<350 \mathrm{GeV}$ ), one can see that the few points with negative $\mu A_{t}$ yield a value of $\operatorname{BR}\left(B_{s} \rightarrow \mu^{+} \mu^{-}\right)$close to the $\mathrm{SM}$ result, whereas the many points with positive $\mu A_{t}$ predict a smaller $\operatorname{BR}\left(B_{s} \rightarrow \mu^{+} \mu^{-}\right)$than in the $\mathrm{SM}$, which are in better agreement with the current experimental central value. These negative corrections to $\operatorname{BR}\left(B_{S} \rightarrow \mu^{+} \mu^{-}\right)$become more sizable for larger values of $\tan \beta \gtrsim 5$. It is interesting to note that predictions for $\operatorname{BR}\left(B_{s} \rightarrow \mu^{+} \mu^{-}\right)$that precisely match the experimental central value are possible over the whole range of $M_{H^{ \pm}}$values displayed in the left plot of Fig. 12.

The decay $B^{+} \rightarrow \tau^{+} v$ is helicity suppressed in the SM. The tree-level exchange of a charged Higgs boson constitutes the dominant MSSM contribution. These contributions can be sizable for large values of $\tan \beta$ and small $M_{H^{ \pm}}$. In the low- $M_{A}$ selection we have small $\tan \beta$ after including the

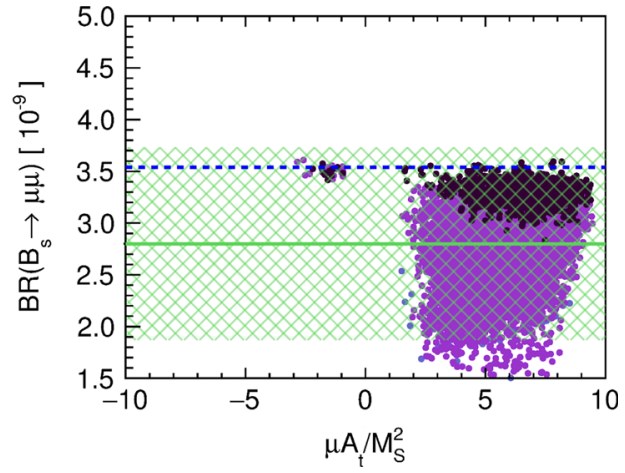

the corresponding experimental measurements and the total $1 \sigma$ uncertainty region, while the SM prediction is indicated by the blue dashed line. The color coding of the displayed points is the same as in Fig. 9

$h / H / A \rightarrow \tau^{+} \tau^{-}$exclusion likelihood and thus the contributions to $\operatorname{BR}\left(B^{+} \rightarrow \tau^{+} \nu\right)$ are small. We have checked that the MSSM predictions are close to the SM value over the entire range of $M_{H^{ \pm}}$covered in our scan.

Finally, Fig. 13 shows the prediction for the SUSY contribution to the anomalous magnetic moment of the muon, $\delta a_{\mu}$, against the first and second generation slepton mass, $m_{\tilde{e}, \tilde{\mu}}$ for all scan points (left) and in the low- $M_{A}$ selection (right). ${ }^{18}$ The MSSM contributions to $a_{\mu}$ consist of smuon-neutralino $\left(\tilde{\mu}^{ \pm}-\tilde{\chi}^{0}\right)$ and sneutrino-chargino $\left(\tilde{v}-\tilde{\chi}^{ \pm}\right)$ loops. Clearly $\delta a_{\mu}$ is strongly correlated with the slepton mass, whereas the correlation with the chargino and neutralino masses (not shown) is less pronounced. In the full scan the favored region extends to large $\tan \beta$ values, implying that sizable contributions to $\delta a_{\mu}$ are possible also for moderately large slepton masses. Parameter points within the $1 \sigma$ region around the desired $\delta a_{\mu}$ value are found all the way up to $m_{\tilde{e}, \tilde{\mu}} \sim 1000 \mathrm{GeV}$ for large $\tan \beta$. In the low- $M_{A}$ selection, $\tan \beta$ is small $(\lesssim 10)$ and therefore the sleptons need to be very light, $\lesssim 300 \mathrm{GeV}$, in order to give a large enough contribution to $\delta a_{\mu}$. This has interesting consequences for the direct SUSY searches, discussed below, where we find that a larger fraction of the preferred points in the alignment region is excluded compared to the decoupling region. This is because SUSY searches with multilepton final states targeting direct stop, gaugino or slepton production yield stronger exclusion if the $1 \mathrm{st} / 2$ nd generation sleptons are light.

\footnotetext{
18 Note that in order to be able to separately analyze the corresponding effects in the fit to the Higgs rates and the fit to $a_{\mu}$, we have chosen to treat $M_{\tilde{\ell}_{1,2}}$ and $M_{\tilde{\ell}_{3}}$ as independent fit parameters in this work. Indeed, light staus can significantly influence the Higgs rates, in particular the $\gamma \gamma$ rate $[19,152,155]$.
} 


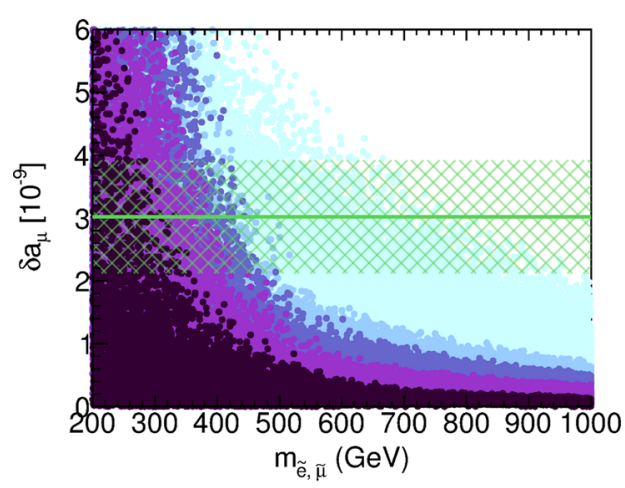

Fig. 13 The SUSY contribution to the anomalous magnetic moment of the muon, $\delta a_{\mu}$, as a function of the (1st and 2nd generation) slepton mass, $m_{\tilde{e}, \tilde{\mu}}$, for the favored points in the fit without taking into account the LEOs, for all scan points (left) and for the low- $M_{A}$ selection, $M_{A}<350 \mathrm{GeV}$ (right). The green line indicates the desired new

\subsubsection{Impact of direct LHC SUSY searches}

As discussed in Sect. 3.3, we have included all relevant $8 \mathrm{TeV}$ SUSY searches in our analysis. ${ }^{19}$ Overall, we find that the LHC SUSY searches impact the validity of the scan points in the light Higgs interpretation considerably. In total $38 \%$ of the $2.4 \times 10^{5}$ tested $p M S S M 8$ parameter points are excluded by the limits from LHC SUSY searches. ${ }^{20}$ Restricting ourselves to the points within the (approximate) 95\% C.L. favored region, LHC SUSY searches exclude $43 \%$ of these points. The impact of the direct LHC SUSY searches is mostly "orthogonal" to the impact of the Higgs constraints in the sense that the direct SUSY searches have no impact on the $\chi^{2}$ profile. This observation can already be inferred from the light gray points of Fig. 4.

This point is further exemplified in the left plots of Fig. 14 in two-dimensional mass planes of the lightest stop, stau, selectron/smuon and neutralino, which are the most important SUSY particles in this context. The impact of the LHC searches for SUSY particles has been tested with CheckMATE for all displayed points. The points excluded by direct SUSY search limits are plotted in pale colors above the points allowed by the direct SUSY searches, which are shown in bright colors. The excluded points are found with a similar distribution as the allowed points in all projections of these mass parameters. The reduced density of excluded points for low values of the lightest neutralino mass, $m_{\tilde{\chi}_{1}^{0}}$, is explained by a reduction of the signal acceptance in most of the searches applied (the $\tilde{t} \tilde{t} \rightarrow 1 \ell+N$ jets $+E_{T}^{\text {miss }}$ search [164] being a notable exception). This is caused by

\footnotetext{
19 CheckMATE-1.2.2 does not yet include the first $13 \mathrm{TeV}$ analyses of ATLAS and CMS. However so far the $13 \mathrm{TeV}$ exclusion limits are comparable (or still weaker) than the $8 \mathrm{TeV}$ limits.

${ }^{20}$ Recall that only points with a $\Delta \chi^{2} \leq 10$ with respect to the BF point are fed into the CheckMATE analysis.
}

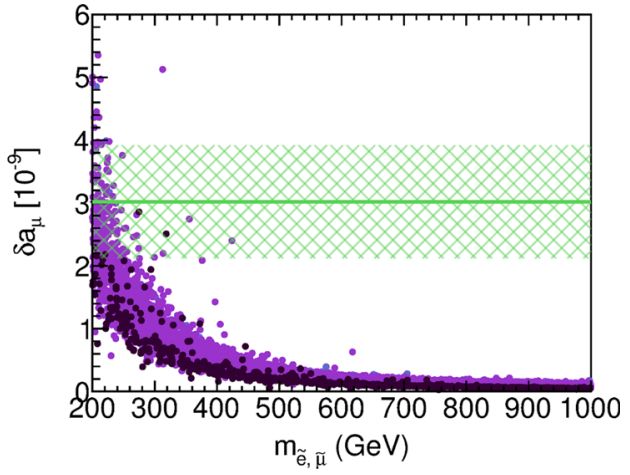

physics contribution needed to achieve agreement with the observed deviation from the SM, and the hatched region corresponds to the $1 \sigma$ experimental uncertainty. The color coding of the displayed points is the same as in Fig. 9

the fact that often $m_{\tilde{\chi}_{1}^{0}} \approx \frac{1}{2} m_{\tilde{\chi}_{1}^{ \pm}} \approx \frac{1}{2} m_{\tilde{\chi}_{2}^{0}}$ due to the assumption of (6). For small neutralino masses the signal acceptance of $\tilde{\chi}^{ \pm} / \tilde{\chi}^{0} \rightarrow\left(W^{ \pm} / Z\right) \chi_{1}^{0}$ final states reduces as the phase space of the decay decreases, $m_{\tilde{\chi}^{0} / \tilde{\chi}^{ \pm}}-m_{\tilde{\chi}_{1}^{0}} \rightarrow M_{W} / M_{Z}$ (see e.g. Fig. 11 of Ref. [168]), yielding reduced final state activity. Furthermore, the missing energy is smaller for small neutralino masses, affecting in particular the signal acceptance of searches for hadronic final states. Nevertheless, we do not find any specific effect of this feature on the allowed Higgs sector phenomenology.

Although the impact of the Higgs data seems orthogonal to the impact of the LHC SUSY searches, the impact of some of the included LEOs, in particular $a_{\mu}$, is not. The latter favors light first/second generation sleptons and light gauginos, which can be probed by searches at the LHC. As a result, we find a significant amount of favored points being excluded by multilepton searches for direct slepton, electroweak gaugino or stop pair production. As already mentioned above, this effect is particularly prominent in the low- $M_{A}$ region, where the requirement of light $1 \mathrm{st} / 2$ nd generation sleptons to accommodate $\delta a_{\mu}$ is much stricter than in the decoupling region (cf. Fig. 13).

For the SUSY exclusion using CheckMATE, only $8 \mathrm{TeV}$ searches and LO cross sections are used with a global $k$ factor of 1.5 (see Sect. 3.3), due to their availability in the applied computer codes (as mentioned above, the version of CheckMATE used for our analysis does not yet include the first limits from ATLAS and CMS searches at $13 \mathrm{TeV}$ ). The results shown in the left plots of Fig. 14 imply that possible limits from upcoming searches at $13 \mathrm{TeV}$ are not expected to significantly alter the Higgs signal interpretation in the pMSSM 8: Due to the described "orthogonality" of the SUSY searches to the Higgs rate constraints, a refinement of the $k$-factor calculation or a further strengthening of the limits, both in terms of rate constraints and in terms of mass reach, are not expected to change the parameter ranges pre- 

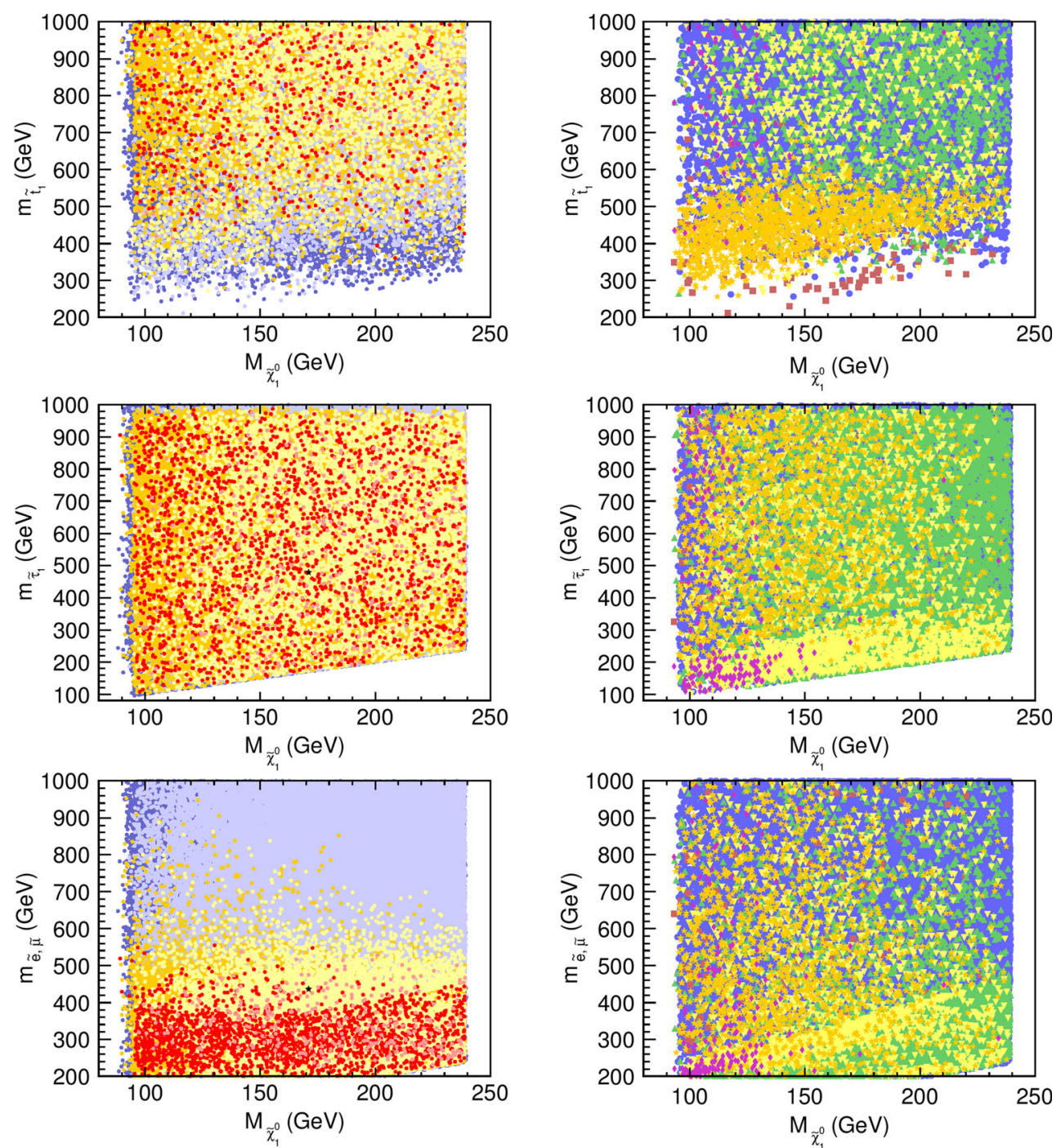

Fig. 14 Impact of 8 TeV LHC SUSY searches on the fit, in dependence of the lightest stop (top row), stau (middle row) and selectron/smuon (bottom row) mass and the lightest neutralino mass. In the three left panels, we compare the CheckMATE allowed (bright colored) and excluded (pale colored) points for all scan points (blue), the (approximate) $95 \%$ C.L. (yellow) and $68 \%$ C.L. (red) preferred points (plotted in this order, with excluded points on top of the allowed points in each step); In the three right panels, we exhibit LHC analyses that

yield the exclusion (in the order of plotting): (i) $N$ jets $+E_{T}^{\text {miss }}$ searches (blue circles) [156,157], (ii) hadronic $\tilde{t} \tilde{t}$ searches with $b$-jets (red squares) [158-161], (iii) $\chi_{1}^{ \pm} \chi_{2}^{0} \rightarrow 3 \ell+E_{T}^{\text {miss }}$ searches (green up-triangles) [162], (iv) $\tilde{t} \tilde{t} \rightarrow 2 \ell+E_{T}^{\text {miss }}$ searches (yellow downtriangle) [163], (v) $\tilde{t} \tilde{t} \rightarrow 1 \ell+N$ jets $+E_{T}^{\text {miss }}$ searches (orange stars) [164], (vi) $\chi_{1}^{ \pm} \chi_{2}^{0}, \tilde{\ell} \tilde{\ell} \rightarrow \ell^{+} \ell^{-}+E_{T}^{\text {miss }}$ searches (magenta diamonds) [165], (vii) $2 \ell+N$ jets $+E_{T}^{\text {miss }}$ (gray plus) $[166,167]$

ferred by the Higgs rate observables significantly. Instead, it can be expected that strengthened SUSY limits will only decrease the point density, thus indicating that parameters need an increasingly refined tuning in order to still find a good fit for the Higgs mass and the Higgs rates.

The sensitivity of the various SUSY searches in the different kinematical regions of the parameter space is shown in the right plots of Fig. 14, where we give for every excluded parameter point the relevant LHC SUSY search (see figure caption for a detailed list and references). In the upper right plot in Fig. 14, hadronic and one-lepton stop searches (red squares and orange stars) can be easily identified to be

the most sensitive searches for $m_{\tilde{t}} \lesssim 500 \mathrm{GeV}$, while for larger masses a more mixed distribution of all searches is observed. Likewise, in the middle and bottom right plots in Fig. 14 it can be seen that the sensitivity of LHC searches for direct electroweak gaugino and slepton production with dilepton final states (magenta diamonds) is centered at low mass parameters for sleptons, staus and neutralinos. Dilepton searches for stop pairs (yellow down-triangles) and multilepton searches for electroweak gauginos (green up-triangles) also provide important constraints at low stau and slepton masses, and in particular remain sensitive at larger neutralino masses. 


\subsection{The heavy Higgs interpretation}

We now consider the more exotic MSSM interpretation where the heavy $\mathcal{C P}$-even Higgs boson is identified as the observed Higgs boson at $125 \mathrm{GeV}$. We recall that this interpretation has a similarly good fit quality as the light Higgs interpretation (see Table 3). As in the light Higgs case we first show the predictions for the Higgs signal rates and their correlations and then discuss the preferred parameter region. After commenting on the impact of the low energy observables and LHC SUSY searches on the fit we conclude this section by discussing the discovery prospects for the other neutral and charged Higgs states in the heavy Higgs interpretation.

\subsubsection{Higgs signal rates}

We show the $\Delta \chi^{2}$ profiles in the most important Higgs signal rates (cf. (48)) in Fig. 15. From the sparseness of the allowed points (blue) in these distributions it is evident that the heavy Higgs interpretation is quite constrained. Moreover, the actual $\chi^{2}$ minimum of all scan points lies signif-
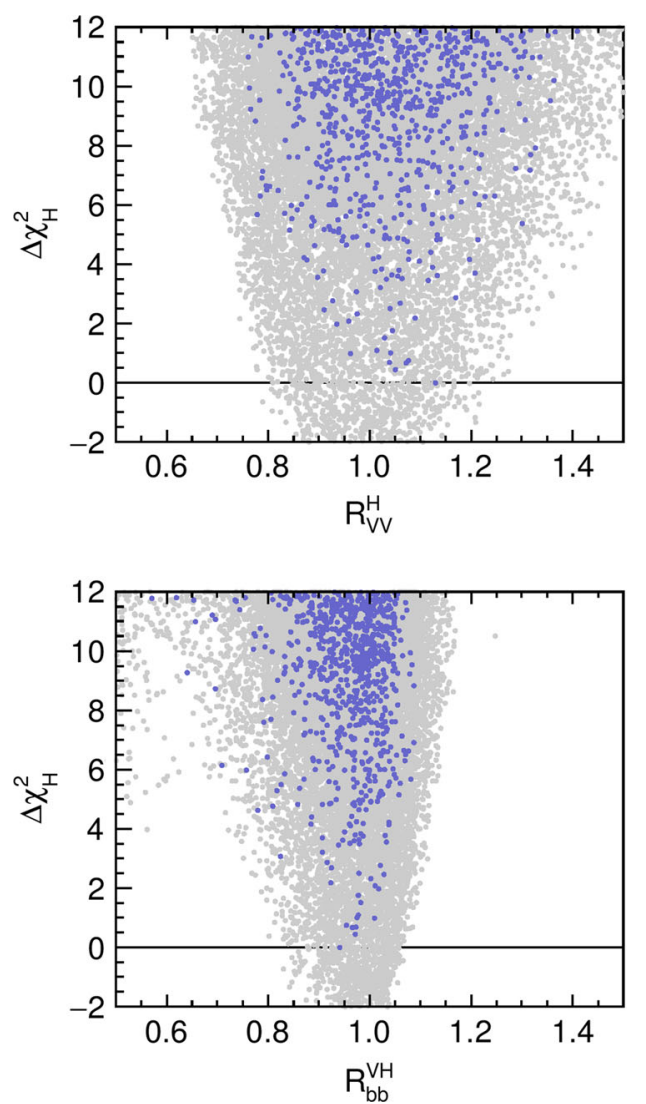

Fig. $15 \Delta \chi^{2}$ distributions for the most important Higgs signal rates (defined in the text) from the complete scan for the heavy Higgs interpretation. The colors show all points in the scan (gray), and points that icantly deeper than the determined best-fit point, however, all points with a lower total $\chi^{2}$ than the best-fit point are excluded by either direct Higgs search limits, SUSY search limits, or other more technical requirements such as the $\mathbf{Z}$ matrix criterion (see Sect. 3.1).

We find the preferred ranges for the Higgs signal rates to be

$R_{V V}^{H} \in[0.95,1.13], \quad R_{\gamma \gamma}^{H} \in[0.81,0.94]$,

$R_{b b}^{V H} \in[0.94,1.03], \quad R_{\tau \tau}^{H} \in[0.78,0.90]$.

The Higgs rates $R_{X X}^{P(H)}$ are defined as in the light Higgs case, see (48), but with $h \leftrightarrow H$. Due to the sparseness of points in the vicinity of the $\chi^{2}$ minimum, these ranges should be taken only as indicative results for the actual $68 \%$ C.L. range (and therefore we also refrain from giving the central values here). Nevertheless, these distributions indicate on the one hand rather good agreement with the SM prediction $\left(R_{X X}^{H}=\right.$ 1) for the $H \rightarrow V V$ and $V H \rightarrow V b \bar{b}$ channels, and on the other hand a modest suppression of the $H \rightarrow \gamma \gamma$ and $H \rightarrow \tau \tau$ signal rates with respect to the SM prediction.
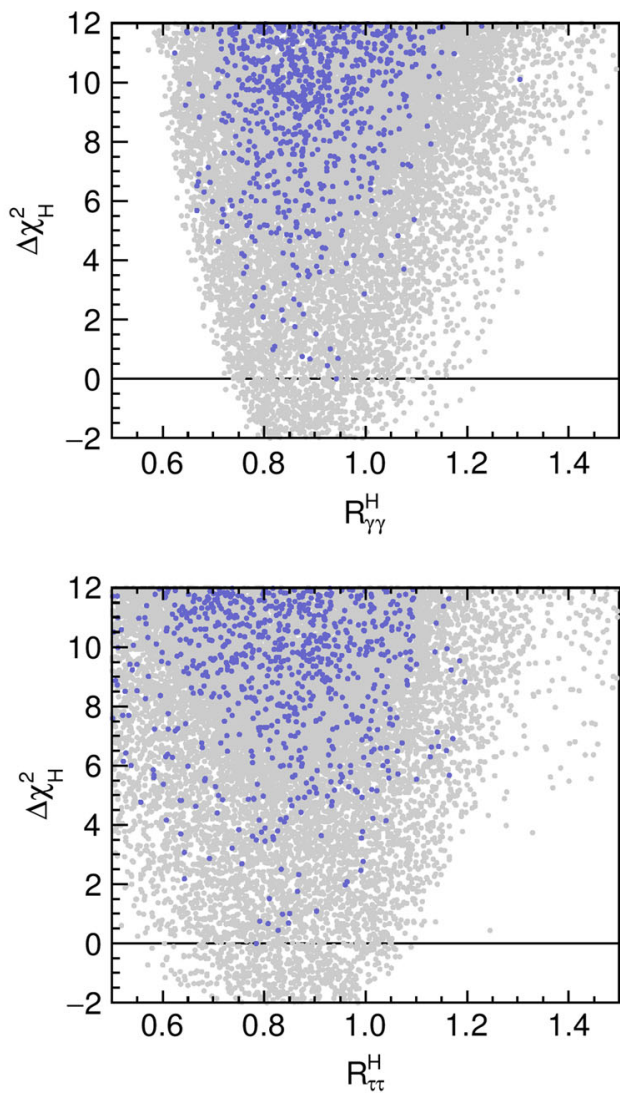

pass the direct constraints from Higgs searches from HiggsBounds (v. 4.2.1) and from LHC sparticle searches from CheckMATE (v.1.2.2) (blue) 

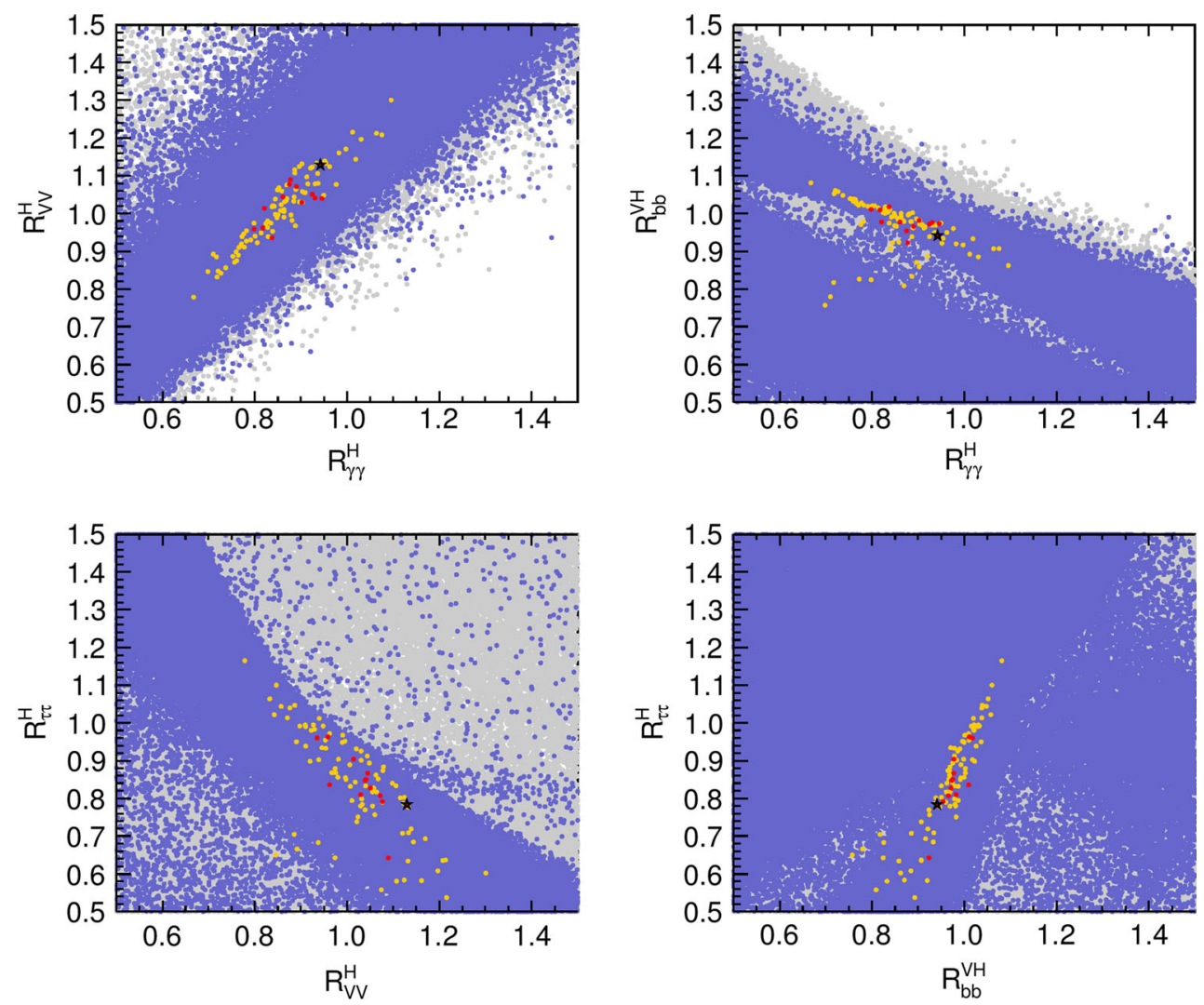

Fig. 16 Correlations between signal rates for the heavy Higgs case. The color coding follows that of Fig. 15, with the addition of the favored regions with $\Delta \chi_{H}^{2}<2.3$ (red) and $\Delta \chi_{H}^{2}<5.99$ (yellow). The best fit point is indicated by a black star

These tendencies appear not only for the most favored points (with $\Delta \chi^{2} \leq 1$ ) but also for the bulk of allowed points with larger $\Delta \chi^{2}$ values. They may thus be tested in the current and upcoming LHC runs.

In Ref. [19] we analyzed two mechanisms for modifying $R_{\gamma \gamma}$. It can be enhanced (suppressed) either via a suppression (enhancement) of the total width, which is dominated by $H \rightarrow b \bar{b}$, or via a directly enhancement (suppression) of the $H \rightarrow \gamma \gamma$ decay width through light charged particles, e.g. the lightest stau. Since we observe $R_{b b}^{V H} \sim 1$ here, the suppression of $R_{\gamma \gamma}^{H}$ is due to a direct suppression of the $H \rightarrow \gamma \gamma$ width induced by SUSY loops in the $H \rightarrow \gamma \gamma$ decay amplitude. Indeed we find for the most favored points $\Gamma(H \rightarrow \gamma \gamma) / \Gamma(H \rightarrow \gamma \gamma)_{\mathrm{SM}} \sim 0.80-0.95$, from loops of a light charged Higgs boson and a moderately light stop, $m_{\tilde{t}_{1}} \sim(350-650) \mathrm{GeV}$, where the stop mixing parameter is close to $X_{t} \sim-1.5 M_{S}$ (see Sect. 4.3.2) [152].

We show some of the correlations of the four Higgs signal rates in Fig. 16. Interestingly, all preferred parameter points have $R_{V V}^{H}>R_{\gamma \gamma}^{H}$, with almost all of them following a strong linear correlation approximately given by $R_{V V}^{H} \approx 0.05+1.23 \cdot R_{\gamma \gamma}^{H}$. Note that in the light Higgs case we observed a similar linear correlation, however, $R_{V V}^{h}<R_{\gamma \gamma}^{h}$ for most of the scan points in that case. Precision determination of the $H \rightarrow V V$ and $H \rightarrow \gamma \gamma$ rates might there- fore help to distinguish between the two interpretations. The other rate correlations, namely $\left(R_{\gamma \gamma}^{H}, R_{b b}^{V H}\right),\left(R_{V V}^{H}, R_{\tau \tau}^{H}\right)$ and $\left(R_{b b}^{V H}, R_{\tau \tau}^{H}\right)$, are very similar to those found for the light Higgs interpretation (cf. Fig. 5). We note that the (approximate) $95 \%$ C.L. region extends over smaller values for the $H \rightarrow \tau^{+} \tau^{-}$rate than in the light Higgs interpretation, down to values of $R_{\tau \tau}^{H} \gtrsim 0.5$.

\subsubsection{Parameter space}

We show the fit results for the heavy Higgs interpretation in Fig. 17 in the $\left(M_{A}, \tan \beta\right)$ plane (left) and the $\left(\mu / M_{S}, A_{t} / M_{S}\right)$ plane (right). The preferred parameter points expand over only a narrow range in the parameters determining the Higgs sector at lowest order, $M_{A} \sim$ $(140,185) \mathrm{GeV}$ and $\tan \beta \sim 6-11$. Compared to our previous results [19], where we found smaller values $M_{A} \sim(110$ 140) $\mathrm{GeV}$ being preferred, the favored parameter region has shifted towards larger $M_{A}$ values, caused by several reasons. Firstly, at small values $M_{A} \lesssim 150 \mathrm{GeV}$ the $\mathcal{C P}$-odd Higgs boson $A$ potentially contributes to the predicted signal rate at $125 \mathrm{GeV}$ in the $\tau^{+} \tau^{-}$channel. ${ }^{21}$ In that case,

\footnotetext{
${ }^{21}$ HiggsSignals automatically adds the signal rates of Higgs bosons that overlap within the combined experimental and theoretical
} 

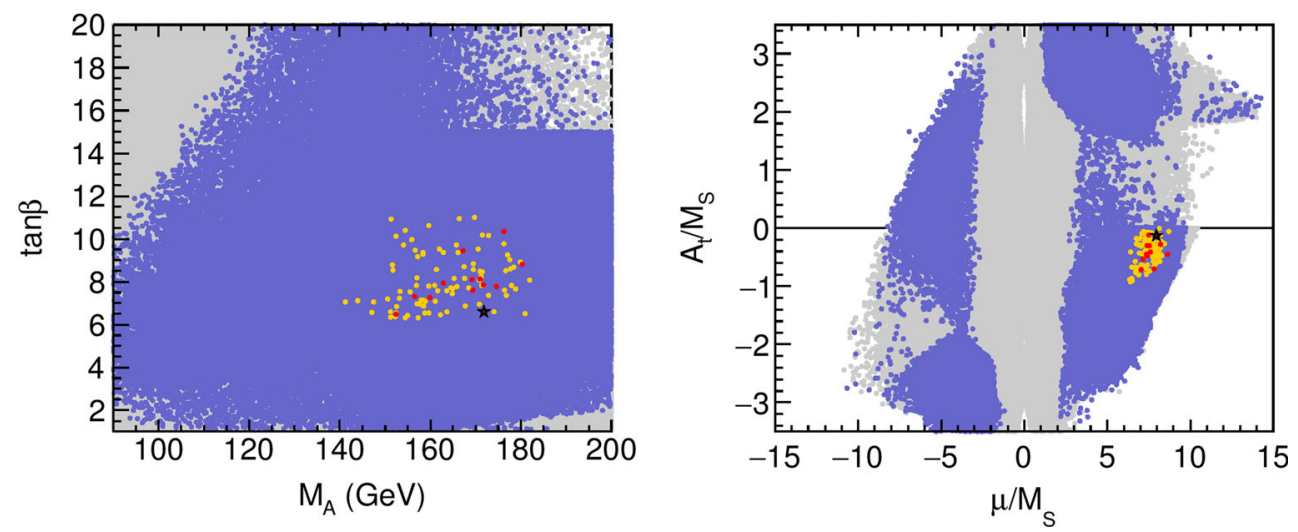

Fig. 17 Preferred parameter regions in the $\left(M_{A}, \tan \beta\right)$ plane (left) and the $\left(\mu / M_{S}, A_{t} / M_{S}\right)$ plane (right) in the heavy Higgs case. The color coding is the same as in Fig. 16

the predicted signal rate would tend to be higher than the total observed $\tau^{+} \tau^{-}$rate, resulting in a larger $\chi^{2}$ from Higgs Signals. In Ref. [19] we also took a possible signal overlap of $H$ and $A$ in the $\tau^{+} \tau^{-}$channel into account; the measurements at that time, however, were not accurate enough to notably affect the fit outcome. Secondly, parameter points with charged Higgs masses $M_{H^{+}}$below $160 \mathrm{GeV}$ are strongly constrained by exclusion limits from LHC searches for a charged Higgs boson in top quark decays, $t \rightarrow H^{+} b$, with successive decay to $\tau$ leptons, $H^{+} \rightarrow \tau^{+} \nu_{\tau}[44,45]$. At tree-level, the $\mathcal{C P}$-odd and charged Higgs masses are related as $M_{H^{ \pm} \text {,tree }}^{2}=M_{A}^{2}+M_{W}^{2}$, thus, these constraints apply in particular at low values $M_{A} \lesssim 140 \mathrm{GeV}$. In Ref. [19] we found good discovery prospects for the heavy Higgs case in $t \rightarrow H^{+} b \rightarrow\left(\tau v_{\tau}\right) b$ searches. Based on the most recent limits from such searches performed by ATLAS and CMS $[44,45]$ the favored parameter regions of Ref. [19] are now excluded and the new preferred parameter space has moved towards larger $M_{A}$ values in the light of the updated limits. Thirdly, another reason for disfavoring $M_{A}$ values below $\sim 150 \mathrm{GeV}$ is the prediction of somewhat too large values of $\operatorname{BR}\left(B \rightarrow X_{s} \gamma\right)$, as will be discussed below.

The distribution of preferred parameter points in the $\left(\mu / M_{S}, A_{t} / M_{S}\right)$ plane, Fig. 17 (right), singles out values of $\mu \sim(6-9) M_{S}$ and $A_{t}$ from $-M_{S}$ to zero. This is due to an interplay of various observables and constraints, as we will outline in the following. Agreement of the heavy Higgs boson $H$ with the LHC Higgs rate measurements requires firstly that $M_{H} \sim 125 \mathrm{GeV}$ and $m_{h}<M_{H}$ (corresponding to $M_{A}<M_{A, c}$, see Sect. 2.2) and secondly that the alignment condition, $Z_{6} v^{2}=0$, is approximately fulfilled. Furthermore, the LHC $H / A \rightarrow \tau^{+} \tau^{-}$constraints impose

\section{Footnote 21 Continued}

mass uncertainties. For most Higgs channels with $\tau^{+} \tau^{-}$final states, the experimental mass resolution is assumed to be $20 \% \cdot m_{H} \approx 25 \mathrm{GeV}$, thus the signals of a $125 \mathrm{GeV}$ heavy Higgs $H$ and a $150 \mathrm{GeV} \mathcal{C P}$-odd Higgs $A$ would be added.

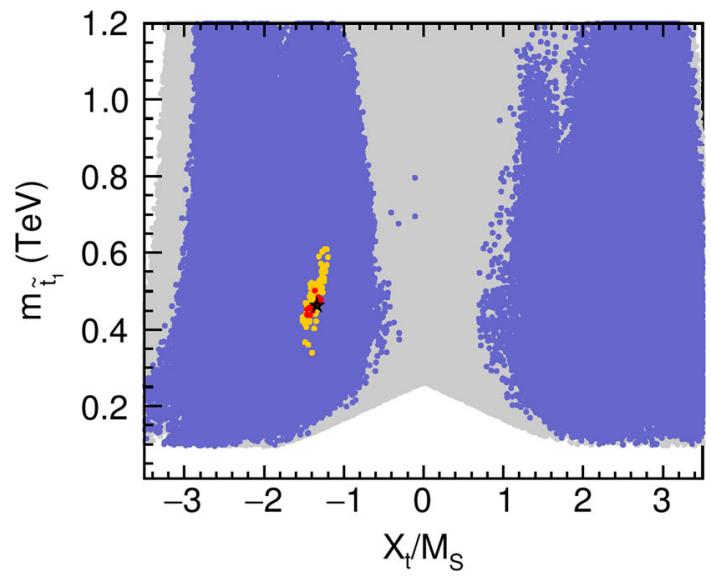

Fig. 18 Preferred parameter regions in the $\left(X_{t} / M_{S}, m_{\tilde{t}_{1}}\right)$ plane in the heavy Higgs case. The color coding is the same as in Fig. 16

that this alignment must occur at not too large $\tan \beta$ values. As we analyzed in detail in Sect. 2.2, these requirements single out small regions of the $\left(\mu, A_{t}\right)$ plane, namely either $\mu / M_{S} \sim A_{t} / M_{S} \approx \pm(3-4)$ or $|\mu| / M_{S} \approx 4-9$ (or even larger) and negative $A_{t}$ with $\left|A_{t}\right| / M_{S} \lesssim 1$. Quite generically, in the heavy Higgs interpretation we have a slightly too high prediction of $\mathrm{BR}\left(B \rightarrow X_{s} \gamma\right)$ due to the light charged Higgs boson. This discrepancy, however, decreases for very large positive values of $\mu / M_{S}$ (similar to the alignment limit in the light Higgs case, cf. Fig. 10). Combining these arguments we find that large positive $\mu$ values, together with rather small negative $A_{t}$ are favored.

In order to avoid problems with vacuum instability, we applied a cut of $|\mu| / M_{S}<3$ in the light Higgs case. Obviously, all our favored points in the heavy Higgs case would be cut away by such a constraint. ${ }^{22}$ However we want to stress

\footnotetext{
22 Taking into account only the parameter points which would survive such a cut $\left(|\mu| / M_{S}<3\right)$, we find a minimum $\chi_{H}^{2}$ of 90.7 for the full fit $(v=85)$, corresponding to a $p$ value of 0.32 . In this case the best-fit region features values of $M_{\tilde{q}_{3}} \sim 1 \mathrm{TeV}$ and $\mu \sim A_{t} \approx(2.5-3) M_{S}$.
} 


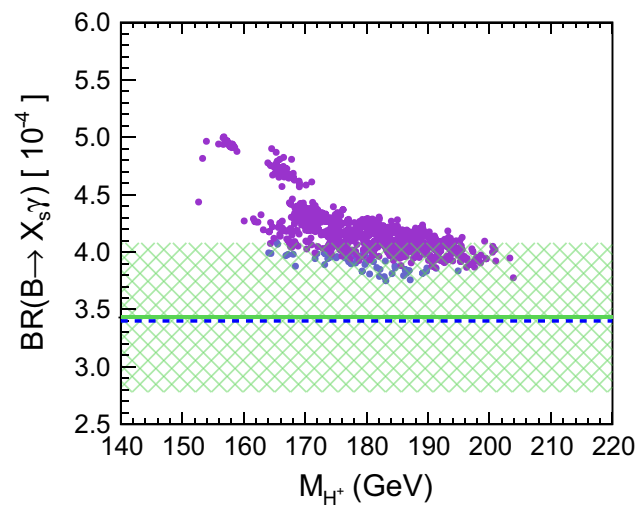

Fig. $19 \mathrm{BR}\left(B \rightarrow X_{s} \gamma\right)$ vs. $M_{H^{ \pm}}$(left) and $\mu / M_{S}$ (right) for the favored points in the heavy Higgs case in the fit without taking into account the LEOs. The green line and hatched region show the experi-

here that this cut only provides an approximate limit. Testing if any of our favored points in the heavy Higgs case are still allowed by vacuum stability would require a more thorough analysis, which is beyond the scope of this paper.

The fact that $A_{t}$ and $\mu$ are predicted to be confined to a narrow range in the heavy Higgs interpretation also results in a definite prediction for $X_{t}$ and a quite small range for the light stop mass, as shown in Fig. 18. The favored region has $X_{t} \sim-1.5 M_{S}$, and the light stop mass is found between 350 and $650 \mathrm{GeV}$ - a prediction that can be tested at the upcoming LHC stop searches. However, note that the upper limit on the light stop mass is a consequence of our restricted scan range, $\mu \leq 5 \mathrm{TeV}$, and the fact that large $\mu / M_{S}$ values are favored.

\subsubsection{Impact of low energy observables}

The discussion of the low energy observables in the heavy Higgs case closely follows the corresponding discussion in the alignment limit of the light Higgs case, see Sect. 4.2.3, as the parameters most relevant for the low energy observables are similar, i.e. we again have small $M_{A}, M_{H^{ \pm}}$and small $\tan \beta$.

Figure 19 shows $\mathrm{BR}\left(B \rightarrow X_{s} \gamma\right)$ as a function of $M_{H^{ \pm}}$ (left plot) and $\mu / M_{S}$ (right plot) for the favored points of the fit to the Higgs signal rates and Higgs mass (including the exclusion likelihood from LEP and LHC searches, but without LEOs). As discussed above the parameter points with $\mu / M_{S} \approx 3-4$ feature $A_{t} \sim \mu$, whereas the points with $\mu / M_{S} \gtrsim 6$ correspond to small and negative $A_{t}$ values. By comparing the two plots in Fig. 19 we can also see that the parameter points with $\mu / M_{S} \sim A_{t} / M_{S} \approx 3-4$ have relatively light charged Higgs masses, $M_{H^{ \pm}}<m_{t}$. Again we find the expected increase of $\operatorname{BR}\left(B \rightarrow X_{s} \gamma\right)$ when going to small charged Higgs masses. Overall, we observe surprisingly good agreement with the experimental measurement.

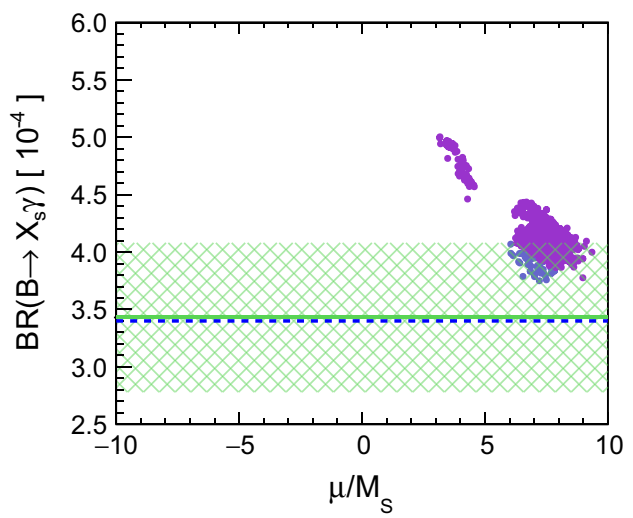

mental measurement and the total $1 \sigma$ uncertainty region, while the SM prediction is indicated by the blue dashed line. The color coding of the displayed points is the same as in Fig. 9

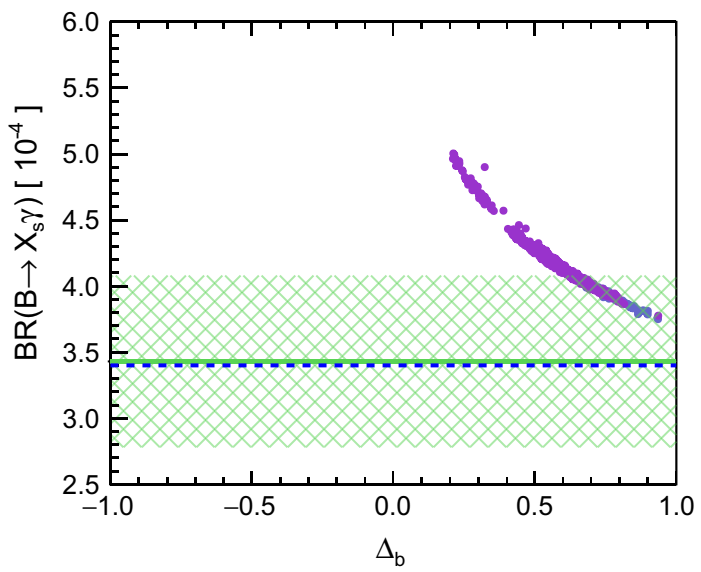

Fig. $20 \operatorname{BR}\left(B \rightarrow X_{s} \gamma\right)$ vs. $\Delta_{b}$ for the favored points in the heavy Higgs case in the fit without taking into account the LEOs. The green and blue line and the hatched region are the same as in Fig. 19. The color coding of the displayed points is the same as in Fig. 9

Even parameter points with a charged Higgs mass below the top quark mass lie mostly within the $2 \sigma$ region of the experimental measurement.

As in the light Higgs case (cf. Fig. 10) we again find that large positive $\mu$ values give the best agreement with the measurement (within the $1 \sigma$ region or just above). However, whereas it takes $\mu / M_{S} \gtrsim 3$ in order that the $\mathrm{BR}\left(B \rightarrow X_{s} \gamma\right)$ prediction be within the $1 \sigma$ region of the measurement in the light Higgs case, here we need much larger values $\mu / M_{S} \gtrsim 6$ to achieve this. Again, this can be understood from the sizable $\Delta_{b}$ corrections to the charged Higgs coupling to top and bottom quarks, as discussed in Sect. 2.3, and shown in Fig. 20, which impressively resembles the relation in Eq. (44).

The $\operatorname{BR}\left(B_{s} \rightarrow \mu^{+} \mu^{-}\right)$prediction is shown in the left plot of Fig. 21 in dependence of $\mu A_{t} / M_{S}^{2}$. We find that the few points with positive $\mu A_{t}$ are in excellent agreement with $\operatorname{BR}\left(B_{s} \rightarrow \mu^{+} \mu^{-}\right)$, whereas for negative $\mu A_{t}$ the predictions are slightly too high, in particular for $\mu A_{t} / M_{S}^{2} \lesssim-5$. Again this can be compared to Fig. 12 where we found a 


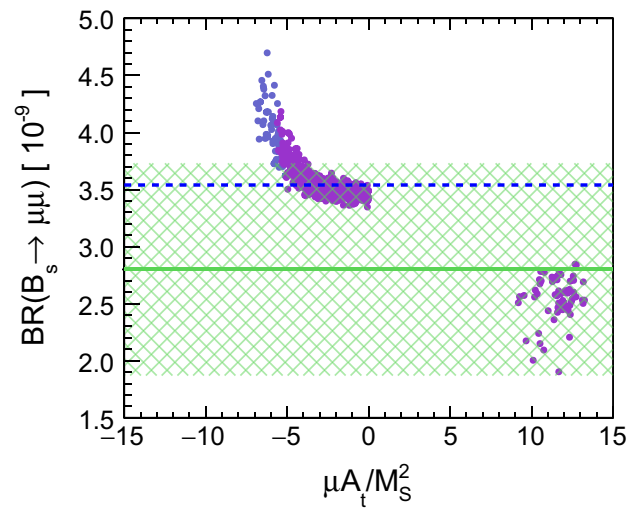

Fig. $21 B_{S} \rightarrow \mu^{+} \mu^{-}$vs. $\mu A_{t} / M_{S}^{2}$ (left) and $\operatorname{BR}\left(B^{+} \rightarrow \tau^{+} \nu\right)$ vs. $M_{H^{ \pm}}(r i g h t)$ for the favored points in the heavy Higgs case in the fit without taking into account the LEOs. The green line and hatched

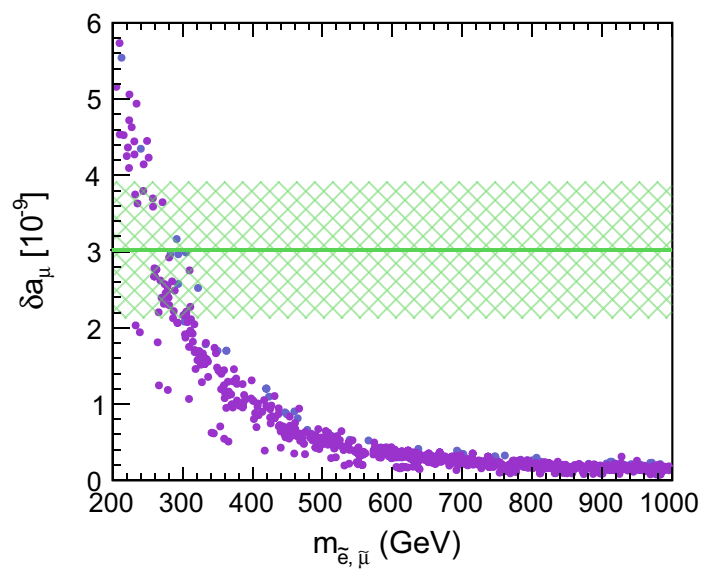

Fig. 22 The SUSY contribution to the anomalous magnetic moment of the muon, $\delta a_{\mu}$, as a function of the (1st and 2 nd generation) slepton mass, $m_{\tilde{e}, \tilde{\mu}}$, for the favored points in the heavy Higgs case in the fit without taking into account the LEOs. The green line and hatched region show the desired new physics contribution needed to achieve agreement with the observed deviation from the SM and the $1 \sigma$ experimental uncertainty. The colors of the displayed points is the same as in Fig. 9

similar result (even though there the typical $\mu A_{t}$ values of the favored points were quite different). Again we find only very small SUSY corrections to $\operatorname{BR}\left(B_{u} \rightarrow \tau v_{\tau}\right)$ (despite the light charged Higgs) due to the small $\tan \beta$ values, as one can see in the right plot of Fig. 21.

The prediction for the SUSY contribution to the anomalous magnetic moment of the muon, $\delta a_{\mu}$, as a function of the slepton mass are shown in Fig. 22. Again we see a very strong correlation, i.e. the value for $\delta a_{\mu}$ is almost entirely determined by the slepton mass. Light slepton masses $\lesssim 300 \mathrm{GeV}$ are favored in the heavy Higgs case.

\subsubsection{Impact of direct LHC SUSY searches}

The comparison of the impact of the direct SUSY searches with the impact of the other constraints qualitatively agrees

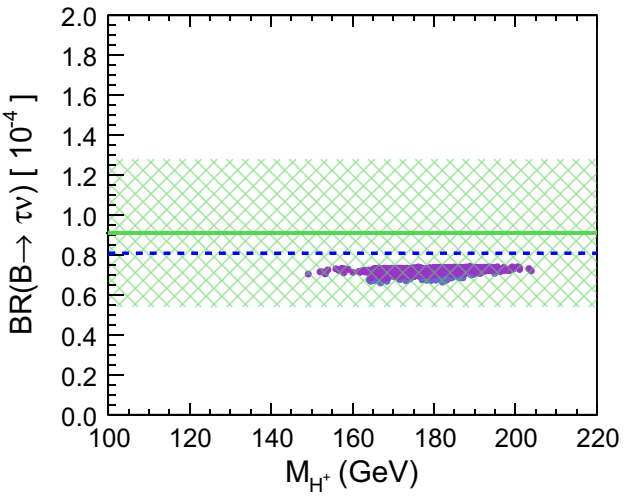

region show the experimental measurements and the total $1 \sigma$ uncertainty region, the SM prediction is indicated by the blue dashed line. The color coding of the displayed points is the same as in Fig. 9

between the light Higgs interpretation (see Sect. 4.3.4) and the heavy Higgs interpretation. Also for the heavy Higgs interpretation, the SUSY search limits are thinning the parameter space in an "orthogonal" way to the Higgs observables and limits from the Higgs searches. We again show the distribution of CheckMATE allowed and excluded points in terms of the most relevant mass parameters in Fig. 23 (left), and indicate the corresponding experimental search that yields the exclusion in Fig. 23 (right). Similar to the light Higgs case, the CheckMATE exclusion is correlated with low-energy constraints, particularly the constraints from the anomalous magnetic moment of the muon. As discussed in the previous section, $a_{\mu}$ strongly favors low slepton masses. Hence we observe a large fraction of favored parameter points excluded by multilepton searches for gaugino pair production (green up-triangles) as long as $M_{2}-m_{\tilde{e}, \tilde{\mu}} \gtrsim 20 \mathrm{GeV}$ is fulfilled. As we have relatively light stops in the preferred region of parameter space, constraints from dilepton searches (yellow down-triangles) as well as hadronic searches with $b$-jets (red squares) targeting stop pair production are also relevant. Overall, LHC SUSY searches exclude only $\sim 5 \%$ of all tested parameter points (i.e. points with $\Delta \chi^{2} \leq 10$ ), but $\sim 65 \%$ of the favored $\left(\Delta \chi^{2}<5.99\right)$ parameter points. This illustrates again the complementarity between the impact of $a_{\mu}$ and the constraints from SUSY searches sensitive to light sleptons.

\subsubsection{Phenomenology of the other Higgs states}

Here we discuss the prospects for the discovery of the other Higgs bosons at the LHC in the heavy Higgs interpretation. We start with the phenomenology of the charged Higgs boson, which is a crucial test of this scenario. In fact, the previous benchmark scenario for the heavy Higgs interpretation [19] has been excluded with limits that have meanwhile been obtained from searches for charged Higgs bosons $[44,45]$. 

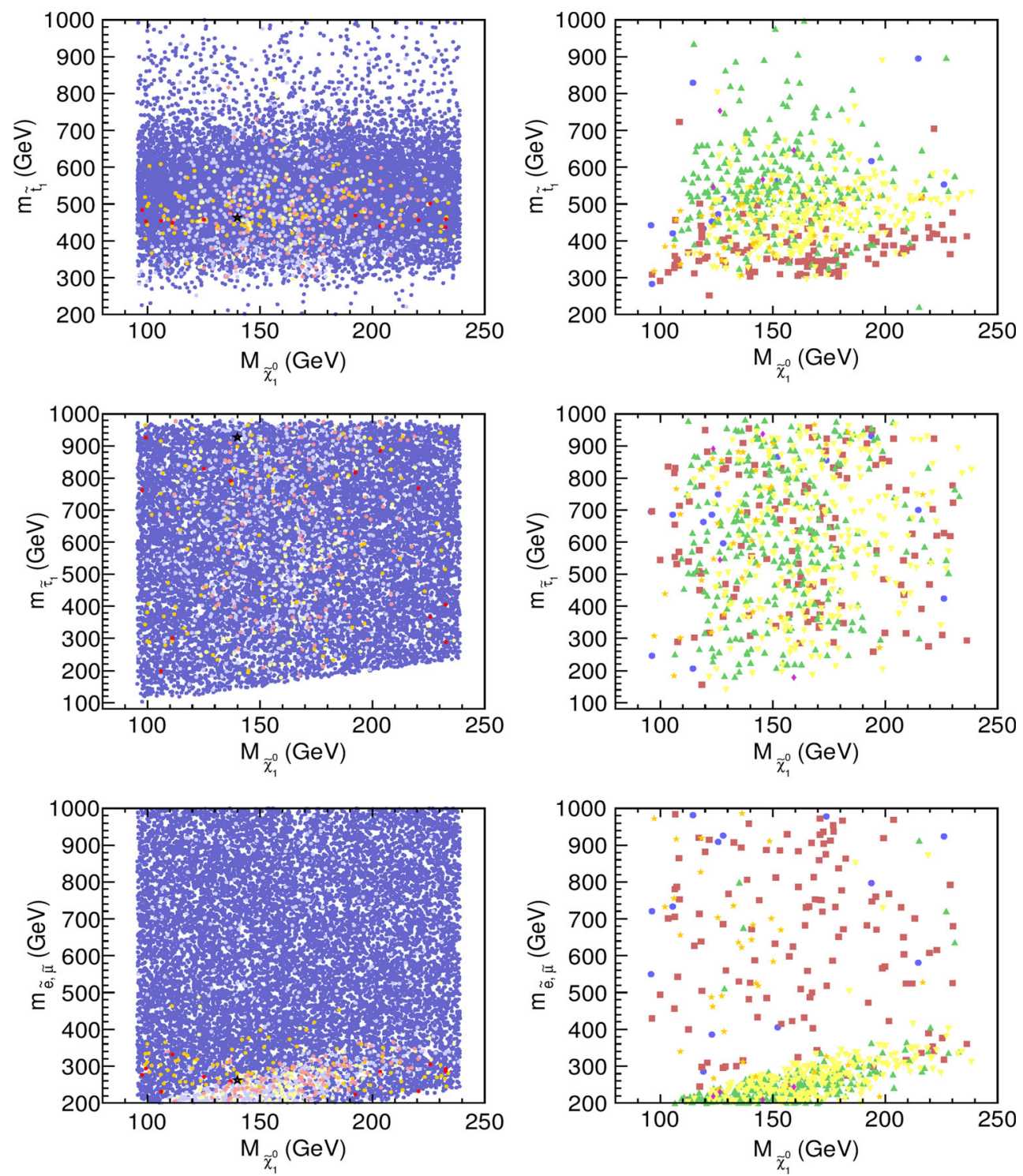

Fig. 23 Impact of $8 \mathrm{TeV}$ LHC SUSY searches on the fit, in dependence of the lightest stop (top row), stau (middle row) and selectron/smuon (bottom row) mass and the lightest neutralino mass. In the three left panels, we compare the CheckMATE allowed (bright colored) and excluded (pale colored) points for all scan points (blue), the $95 \%$ C.L. (yellow) and $68 \%$ C.L. (red) preferred points (plotted in this order, with excluded points on top of the allowed points in each step); In the three right panels, we exhibit LHC analyses that yield the

exclusion (in the order of plotting): (i) $N$ jets $+E_{T}^{\text {miss }}$ searches (blue circles) $[156,157]$, (ii) hadronic $\tilde{t} \tilde{t}$ searches with $b$-jets (red squares) [158161], (iii) $\chi_{1}^{ \pm} \chi_{2}^{0} \rightarrow 3 \ell+E_{T}^{\text {miss }}$ searches (green up-triangles) [162], (iv) $\tilde{t} \tilde{t} \rightarrow 2 \ell+E_{T}^{\text {miss }}$ searches (yellow down-triangle) [163], (v) $\tilde{t} \tilde{t} \rightarrow 1 \ell+N$ jets $+E_{T}^{\text {miss }}$ searches (orange stars) [164], (vi) $\chi_{1}^{ \pm} \chi_{2}^{0}, \tilde{\ell} \tilde{\ell} \rightarrow \ell^{+} \ell^{-}+E_{T}^{\text {miss }}$ searches (magenta diamonds) [165], (vii) $2 \ell+N$ jets $+E_{T}^{\text {miss }}$ (gray plus) $[166,167]$

In Fig. 24 we show the rate for the main production and decay channel of a light charged Higgs boson with $M_{H^{ \pm}}<$ $m_{t}, \mathrm{BR}\left(t \rightarrow H^{ \pm} b\right) \times \mathrm{BR}\left(H^{ \pm} \rightarrow \tau v_{\tau}\right)$, as a function of $M_{H^{ \pm}}$. The impact of the current limits from charged Higgs searches in this channel $[44,45]$ can be seen by the gray area in Fig. 24, cutting out the region with $M_{H^{ \pm}}<160 \mathrm{GeV}$ and $\mathrm{BR}\left(t \rightarrow H^{ \pm} b \rightarrow \tau v_{\tau} b\right) \gtrsim(2-4) \times 10^{-3}$. Only a few favored points (and none of the most favored points) have $M_{H^{ \pm}}<m_{t}$ and are therefore displayed in Fig. 24. As one can see, these points have charged Higgs masses close to the top

quark mass and thus $\mathrm{BR}\left(t \rightarrow H^{ \pm} b\right) \times \mathrm{BR}\left(H^{ \pm} \rightarrow \tau v_{\tau}\right)$ is strongly suppressed due to the limited phase space of the top quark decay. Also the decay $\operatorname{BR}\left(H^{ \pm} \rightarrow \tau v_{\tau}\right)$ is suppressed by the competing decay $H^{ \pm} \rightarrow h W^{ \pm}$, which is open for most of these favored points (we will discuss the $m_{h}$ range of the favored points below). Consequently it is very difficult to detect charged Higgs bosons in this mass range in the $t \rightarrow H^{+} b \rightarrow\left(\tau^{+} v_{\tau}\right) b$ channel at the LHC.

Many of our favored and most favored points have $M_{H^{ \pm}}>$ $m_{t}$ (and are thus not visible in Fig. 24). Charged Higgs 


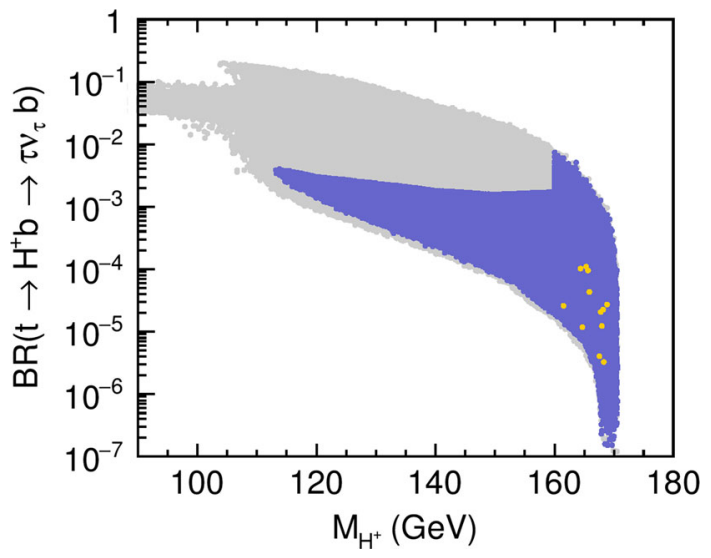

Fig. 24 Branching ratio of the top quark decay into a charged Higgs boson and a bottom quark, with the successive decay of the charged Higgs boson into a tau lepton and neutrino, in the heavy Higgs case. The color coding is the same as in Fig. 16

bosons with masses above the top quark mass are searched for in the $p p \rightarrow t H^{ \pm}$production channel with $H^{ \pm} \rightarrow \tau v_{\tau}$ $[44,45,169]$ or $H^{ \pm} \rightarrow t b[45,170]$. These searches, although concentrating on the charged Higgs mass region that is relevant for the heavy Higgs interpretation, are not yet sensitive to constrain the favored parameter space. However, they will become more sensitive with increasing integrated luminosity. Furthermore, we emphasize again that the decay $H^{ \pm} \rightarrow h W^{ \pm}$is possible and unsuppressed in large parts of the parameter space, but currently not directly searched for at the LHC. In Sect. 4.4 we will present specific benchmark scenarios, inspired by our best-fit point in the heavy Higgs case, that can be employed to study the sensitivity of these searches.

We will now turn to the discussion of the phenomenology of the light $\mathcal{C P}$-even Higgs boson, $h$, in the preferred parameter region in the heavy Higgs case. The light $\mathcal{C P}$-even Higgs boson has a mass in the range (20-90) $\mathrm{GeV}$ and a strongly reduced coupling to vector bosons. This is shown in the top left plot of Fig. 25, where the squared coupling $g_{h V V}^{2}$ is displayed, normalized to the corresponding coupling in the SM with the same value of the Higgs boson mass. One can see that the squared coupling is reduced by a factor of $10^{3}$ or more with respect to the $\mathrm{SM}$, as the heavy $\mathcal{C P}$ even Higgs boson $H$ in this scenario acquires the coupling to vector bosons with approximately SM Higgs strength. This results in a strongly reduced cross section for the LEP Higgs-Strahlung process, $e^{+} e^{-} \rightarrow Z h$. Consequently, the light Higgs boson in this case would have escaped detection in corresponding LEP Higgs searches. The limits from the Higgs searches at LEP occur for higher values of the relative squared coupling $g_{h V V}^{2}$ and are not visible in this plot.

The reduced light Higgs coupling to vector bosons furthermore leads to a reduced rate of the $h \rightarrow \gamma \gamma$ decay, which happens through a $W$-boson loop (amongst other contribut- ing diagrams). In contrast, the light Higgs coupling to gluons is up to ten times stronger than the SM Higgs boson coupling at very low light Higgs masses, $M_{h}$, as shown in the center left plot of Fig. 25, where the (SM normalized) squared light Higgs-gluon coupling, $g_{h g g}^{2}$, is shown in dependence of $M_{h}$. This results in an abundant production of the light $\mathcal{C P}$-even Higgs boson via gluon fusion. The resulting LHC cross section for $g g \rightarrow h$ (at $8 \mathrm{TeV}$ ) with subsequent decay $h \rightarrow \gamma \gamma$ is shown in the bottom left plot of Fig. 25. Limits from LHC searches in this channel [171] have been taken into account in our analysis (using HiggsBounds). Their effect can be seen in this plot as the gray excluded region above $\sim 0.1$ pb. Clearly these searches are currently very far from being sensitive to detect the light Higgs boson in this scenario.

The light Higgs boson predominantly decays to bottom quarks $(\sim(70-80) \%$ of the time) or tau leptons $(\sim(15-30) \%$ of the time), as shown in the top and middle right plots in Fig. 25, respectively. Given the mass range $20 \leq M_{h} \leq$ $90 \mathrm{GeV}$ we expect direct LHC searches in both channels to be rather challenging, given the huge SM background and the difficulty to trigger the events. The majority of the favored points have $M_{h}>M_{H} / 2$, however we also observe some favored points with lower $M_{h}$ (down to $20 \mathrm{GeV}$ ) for which the Higgs-to-Higgs decay channel $H \rightarrow h h$ is kinematically open. If this decay rate is sizable the rates of heavy Higgs $H$ decays to SM particle final states can be significantly affected. We find that for our preferred points the branching ratio of this decay is at most $\sim 20 \%$, but for most points $\lesssim 10 \%$, as shown in the bottom right plot of Fig. 25. Branching fractions of this size lead to only moderate changes of the Higgs decay rates to SM particles. Consequently we still find a good fit in this scenario even for the case $M_{h}<M_{H} / 2$ (the allowed points for $M_{h}>M_{H} / 2$ with vanishing branching ratio of $H \rightarrow h h$ are not visible in the plot).

The $\mathcal{C P}$-odd Higgs boson has a mass between $140 \mathrm{GeV}$ and $185 \mathrm{GeV}$, as shown already in Fig. 17, for $\tan \beta$ in the range between 6 and 11. Consequently, in this scenario it should signify itself with higher luminosity at the LHC in $A \rightarrow \tau \tau$ searches. At the lower end of its mass range it might be visible possibly as an enhanced decay rate of the SM-like Higgs boson due to the limited mass resolution. At the higher end of its mass range it would appear as a new resonance decaying to $\tau^{+} \tau^{-}$.

\subsection{Updated $l o w-M_{H}$ benchmark scenarios}

In the previous section we demonstrated that the heavy Higgs interpretation in which the heavy $\mathcal{C P}$-even Higgs boson is identified with the observed signal [7,34-37] is still a viable scenario. While the original low- $M_{H}$ benchmark scenario [43] has meanwhile been ruled out by ATLAS and CMS via the search for a light charged Higgs boson in top quark decays $[44,45]$, we showed that viable realizations 

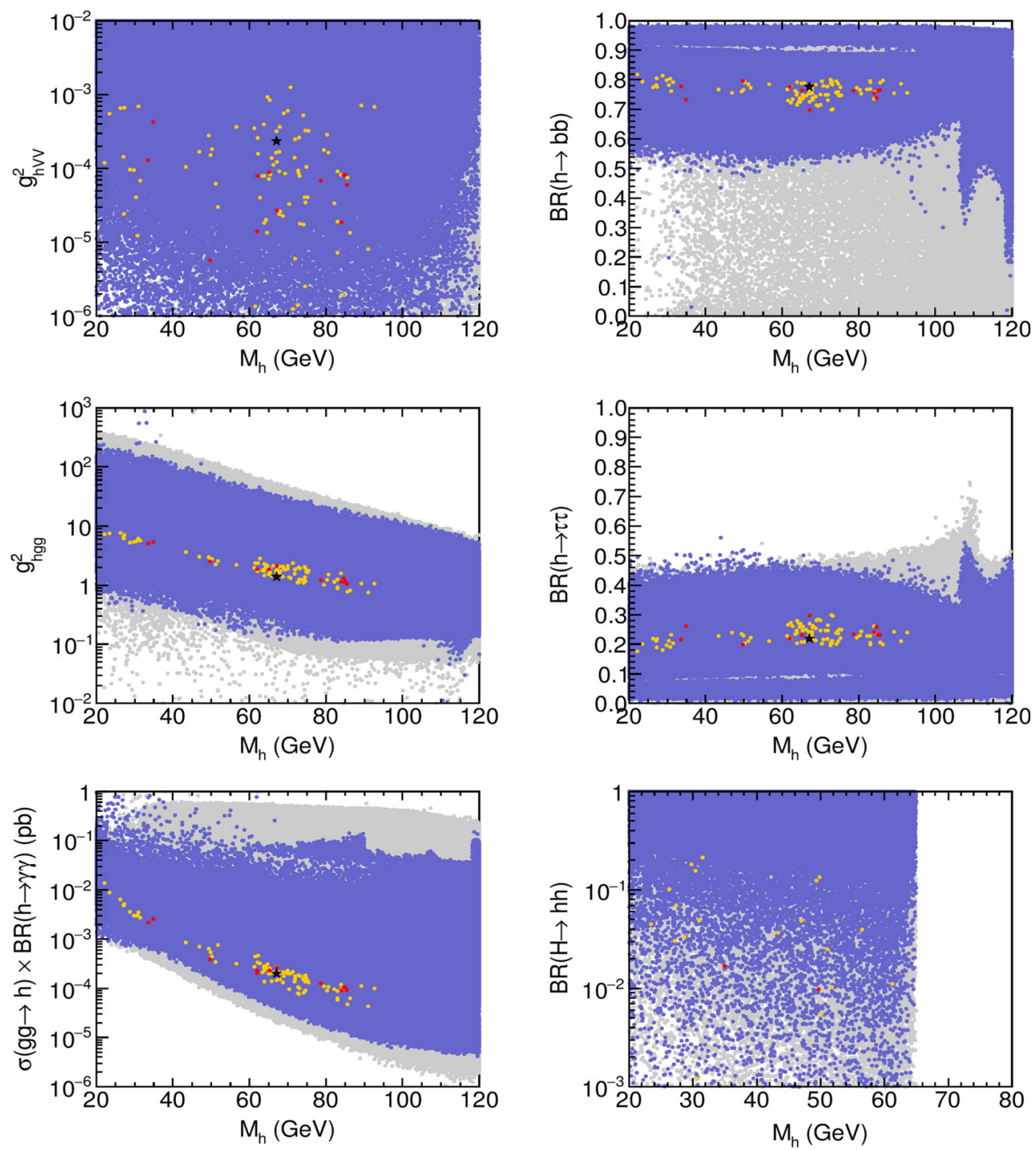

Fig. 25 Light Higgs boson $(h)$ phenomenology, in dependence of the light Higgs mass $M_{h}$, in the heavy Higgs interpretation: (SM normalized) squared $h V V$ coupling, $g_{h V V}^{2}$, (top left) and $h g g$ coupling, $g_{h g g}^{2}$, (middle left), LHC $8 \mathrm{TeV}$ signal rate for the process $g g \rightarrow h \rightarrow \gamma \gamma$

(bottom left), branching fractions for the decays $h \rightarrow b \bar{b}$ (top right), $h \rightarrow \tau^{+} \tau^{-}$(middle right) and the Higgs-to-Higgs decay $H \rightarrow h h$ (bottom right). The color coding is the same as in Fig. 16

of the heavy Higgs interpretation exist outside the parameter region where these searches are sensitive. In this section we define new versions of the $l o w-M_{H}$ benchmark scenario that are valid after taking into account all current experimental constraints. They are inspired by the best-fit point found in our global analysis of the heavy Higgs case, see Table 5, however, we slightly increased the stop mass scale (while roughly retaining the preferred $\mu$ to $M_{S}$ ratio) in order to evade potential exclusion limits on the stop mass from the upcoming $13 \mathrm{TeV}$ LHC results. These new scenarios could provide a useful benchmark for the ongoing light charged Higgs boson searches in the MSSM. In particular, they exhibit the not-yet-sought-for MSSM decay signature $H^{+} \rightarrow W^{+} h$ and, in some parameter regions, even the decay $H^{+} \rightarrow W^{+} H$.

We define three different scenarios, which we call low $-M_{H}^{\text {alt }}$ in order to distinguish them from the previous low $-M_{H}$ benchmark scenario [43]. The first two $l o w-M_{H}^{\text {alt }}$ scenarios follow the original idea in Ref. [43] and fix a "heavy" Higgs boson mass, in this case the charged Higgs boson mass, $M_{H^{ \pm}}$, to a certain value, whereas $\mu$ and $\tan \beta$ are taken as free parameters. We suggest two variants given by different choices of $M_{H^{ \pm}}$below (low $-M_{H}^{\text {alt- }}$ ) and above $\left(\right.$ low $\left.-M_{H}^{\text {alt }+}\right)$ the top quark mass, $m_{t}$. The second scenario fixes $\mu$ and explores the $\left(M_{H^{ \pm}}, \tan \beta\right)$ plane. This scenario may be utilized for charged Higgs boson searches in the relevant mass range. The parameters of these benchmark scenarios are given in Table 6.

In the following we discuss the compatibility of these benchmark planes with the current experimental constraints. 
Table 6 Parameters of the updated low- $M_{H}$ benchmark scenarios. All parameters are given in the on-shell (OS) definition. The lower row gives the fixed parameters that are common to all three benchmark scenarios. $M_{1}$ is fixed via (6)

\begin{tabular}{llll}
\hline Benchmark scenario & $M_{H^{ \pm}}(\mathrm{GeV})$ & $\mu(\mathrm{GeV})$ & $\tan \beta$ \\
\hline low $-M_{H}^{\text {alt- }}$ & 155 & $3800-6500$ & $4-9$ \\
low $-M_{H}^{\text {alt }}$ & 185 & $4800-7000$ & $4-9$ \\
low $-M_{H}^{\text {alt }}$ & $140-220$ & 6000 & $4-9$
\end{tabular}

Fixed parameters: $\quad m_{t}=173.2 \mathrm{GeV}, \quad A_{t}=A_{\tau}=A_{b}=-70 \mathrm{GeV}, M_{2}=300 \mathrm{GeV}$,

$M_{\tilde{q}_{L}}=M_{\tilde{q}_{R}}=1500 \mathrm{GeV}(q=c, s, u, d), m_{\tilde{g}}=1500 \mathrm{GeV}$,

$M_{\tilde{q}_{3}}=750 \mathrm{GeV}, \quad M_{\tilde{\ell}_{1,2}}=250 \mathrm{GeV}, \quad M_{\tilde{\ell}_{3}}=500 \mathrm{GeV}$
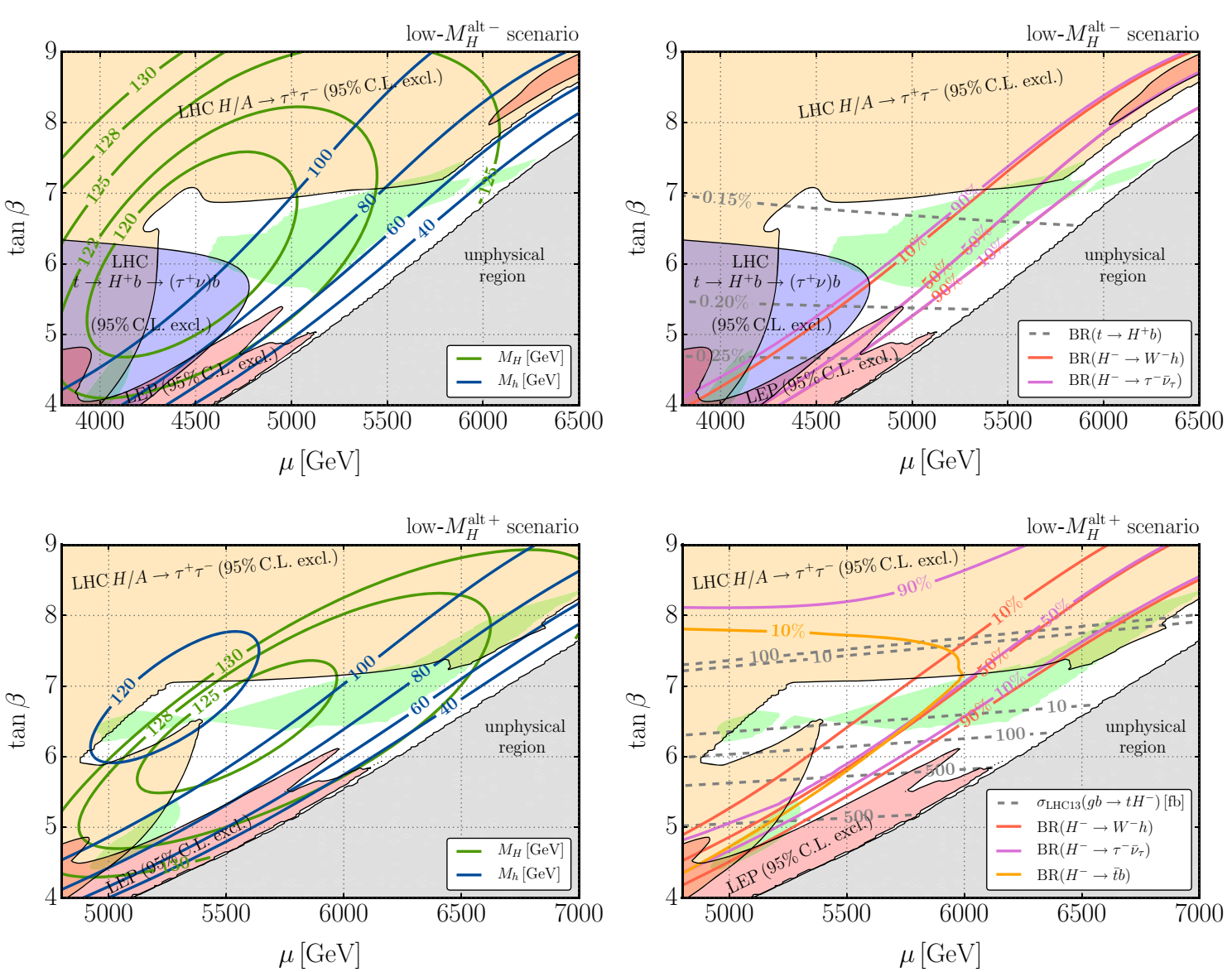

Fig. 26 The $l o w-M_{H}^{\text {alt }-}$ and $l o w-M_{H}^{\text {alt }+}$ benchmark scenarios in the $(\mu, \tan \beta)$ plane with $M_{H^{ \pm}}=155 \mathrm{GeV}$ (upper row), and with $M_{H^{ \pm}}=185 \mathrm{GeV}$ (lower row), respectively. The red, orange and blue regions are disfavoured at the 95\% C.L. by LEP light Higgs $h$ searches [13], LHC $H / A \rightarrow \tau^{+} \tau^{-}$searches [143,144] and LHC $t \rightarrow H^{+} b \rightarrow(\tau \nu) b$ searches $[44,45]$, respectively. The green area indicates parameter regions that are compatible with the Higgs signal

In Fig. 26 we present the results in the $(\mu, \tan \beta)$ plane in the low $-M_{H}^{\text {alt- }}$ scenario with $M_{H^{ \pm}}=155 \mathrm{GeV}$ (upper row) and in the low $-M_{H}^{\text {alt+ }}$ scenario with $M_{H^{ \pm}}=185 \mathrm{GeV}$ (lower row). The blue, red and orange areas indicate the $95 \%$ C.L. excluded regions by LHC $t \rightarrow H^{+} b \rightarrow(\tau \nu) b$ searches [44,45], LEP light Higgs searches [13] (mostly from the $e^{+} e^{-} \rightarrow Z h \rightarrow Z(b \bar{b})$ channel) and LHC $H / A \rightarrow$ $\tau^{+} \tau^{-}$searches [143,144], respectively. For the latter two we again employ the $\chi^{2}$ implementation of these results

(at $\sim 95 \%$ C.L., see text for details), unphysical regions are displayed in gray (see text). In the two left panels, contour lines indicate the Higgs masses $M_{h}$ and $M_{H}$ (in $\mathrm{GeV}$ ). In the two right panels, contours lines indicate the charged Higgs branching ratios, as well as the branching ratio for the top quark decay $t \rightarrow H^{+} b$ (upper row) or the $13 \mathrm{TeV}$ LHC cross section for charged Higgs production in association with a top quark, $\sigma_{\mathrm{LHC} 13}\left(\mathrm{~g} b \rightarrow t \mathrm{H}^{+}\right)$(in fb) $[172,173]$ (lower row)

in HiggsBounds, see Sect. 3.3, and define the 95\% C.L. excluded region by $\Delta \chi^{2} \geq 6.0$ (given the two free parameters of the model). Moreover, we indicate the regions compatible with the Higgs signal based on the total $\chi^{2}$ constructed from Higgs signal rates, Higgs mass and the two exclusion likelihoods from LEP light Higgs searches and LHC $H / A \rightarrow$ $\tau^{+} \tau^{-}$searches. The green area indicates where the $p$ value - estimated from this total $\chi^{2}$ value under the assumption of independent and Gaussian observables - is above 5\%. 


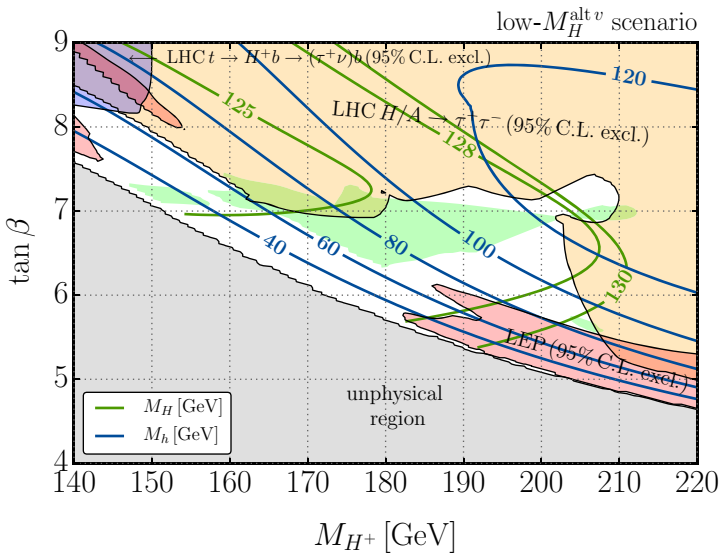

Fig. 27 The $l o w-M_{H}^{\text {alt v }}$ benchmark scenario in the $\left(M_{H^{ \pm}}, \tan \beta\right)$ plane (with $\mu=6000 \mathrm{GeV}$ ). The colored regions follow the definitions in Fig. 26. In the left panel, contour lines indicate the Higgs masses $M_{h}$ and $M_{H}$ (in $\mathrm{GeV}$ ). In the right panel, contours lines indicate the charged

In most of the parameter region of the $l o w-M_{H}^{\text {alt- }}$ and low $-M_{H}^{\text {alt+ }}$ scenarios, the heavy Higgs mass, $M_{H}$, ranges between $120 \mathrm{GeV}$ and $130 \mathrm{GeV}$, whereas the light Higgs mass, $M_{h}$, varies between $\sim 0 \mathrm{GeV}$ (at the edge of the unphysical, gray region) and $120 \mathrm{GeV} \cdot{ }^{23}$ In the $l o w-M_{H}^{\text {alt- }}$ $\left(\right.$ low $-M_{H}^{\text {alt+ }}$ ) scenario the $\mathcal{C} \mathcal{P}$-odd Higgs mass is $M_{A} \sim$ 137 (169) $\mathrm{GeV}$ (within a few $\mathrm{GeV}$ ).

In the low $-M_{H}^{\text {alt }-}$ scenario the branching fraction for the top quark decay into a charged Higgs boson, $t \rightarrow H^{+} b$, ranges between $\sim 0.1$ and $0.25 \%$. The charged Higgs $H^{+}$successively decays either to $\tau^{+} \nu_{\tau}$ or $W^{+} h$, where the branching ratios are highly dependent on the kinematical phase space of the $H^{+} \rightarrow W^{+} h$ decay, and thus on the light Higgs mass, $M_{h}$. Either decay can be completely dominating while the other is suppressed, depending on the parameter space. The charged Higgs boson decay $\mathrm{H}^{+} \rightarrow c \bar{s}$ is negligible.

In the low $-M_{H}^{\text {alt }+}$ scenario, the charged Higgs boson is predominantly produced in association with a top quark via $g b \rightarrow t H^{ \pm}$. The charged Higgs boson branching fractions in the $l o w-M_{H}^{\text {alt }+}$ scenario are very similar to the $l o w-M_{\underline{H}}^{\text {alt }-}$ scenario, with the exception that the decay $H^{+} \rightarrow t \bar{b}$ is present. However, its decay rate amounts to at most $\sim 20 \%$ (at the low end of the $\mu$ range). An interesting situation in this benchmark scenario occurs in the region around $\mu \sim$ (5.0-5.5) $\mathrm{TeV}$ and $\tan \beta \sim 6-7$, where both the light and heavy Higgs boson have masses between 120 and $130 \mathrm{GeV}$. In our analysis, part of this region is even compatible with the Higgs signal and at the same time not directly excluded by limits from Higgs searches, i.e. this part of the parameter space gives rise to two $\mathcal{C P}$-even Higgs bosons close to $125 \mathrm{GeV}$. A dedicated experimental analysis of such a sce-

\footnotetext{
${ }^{23}$ In the evaluation of these benchmark scenarios, the two-loop corrections to the relation between $M_{A}$ and $M_{H^{ \pm}}$have been omitted. Taking them into account will lead to a slight shift in the $M_{h}$ prediction.
}

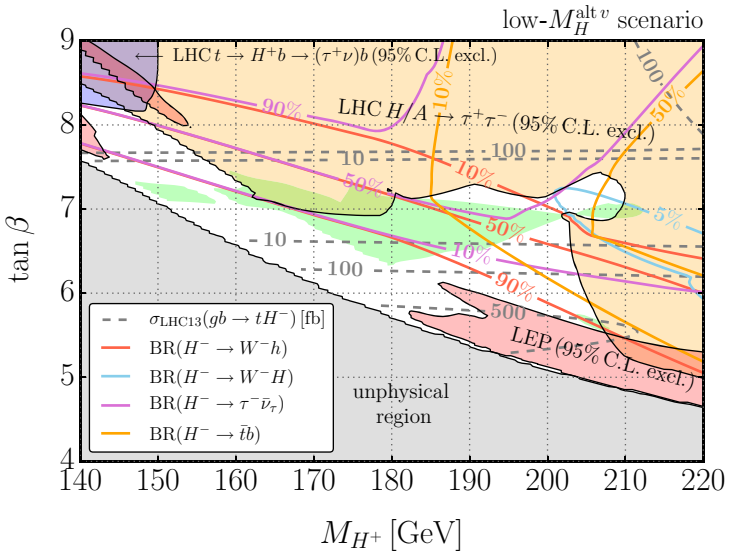

Higgs branching ratios and the $13 \mathrm{TeV}$ LHC cross section for charged Higgs production in association with a top quark, $\sigma_{\mathrm{LHC13}}\left(g b \rightarrow t H^{+}\right)$ (in $\mathrm{fb}$ )

nario, taking into account also interference effects between the two nearly mass-degenerate Higgs bosons, see Ref. [97], would be desirable.

The low $-M_{H}^{\text {alt v }}$ benchmark scenario is illustrated in Fig. 27. Here it can nicely be seen that the limit of alignment without decoupling occurs roughly at $\tan \beta \sim 7$, as the green area is centered around this value. The charged Higgs phenomenology is quite rich: at lower charged Higgs masses, $M_{H^{ \pm}} \lesssim 180 \mathrm{GeV}$, the decay modes $H^{+} \rightarrow \tau^{+} v_{\tau}$ and $H^{+} \rightarrow W^{+} h$ completely dominate. At larger $M_{H^{ \pm}}$, the decay mode $H^{+} \rightarrow t \bar{b}$ and even $H^{+} \rightarrow W^{+} H$ become non-negligible, albeit they remain small for most of the unexcluded parameter space.

The best prospects for exploring these scenarios may still be via LHC searches for $H / A \rightarrow \tau^{+} \tau^{-}$. However, these benchmark scenarios will hopefully also provide useful guidance for upcoming charged Higgs searches and in particular motivate searches for new charged Higgs signatures such as $H^{ \pm} \rightarrow W^{ \pm} h$, with the light Higgs $h$ decaying into bottom quark or tau lepton pairs, $h \rightarrow b \bar{b}, \tau^{+} \tau^{-}$. Excluding these scenarios, which appear to be cornered from all sides, with the upcoming searches at the LHC would strongly restrict the heavy Higgs interpretation in the MSSM, approaching an exclusion of this interesting possibility, whose phenomenology drastically differs from the most commonly considered light Higgs scenario.

\section{Conclusions and outlook}

We have analyzed the compatibility of the phenomenological Minimal Supersymmetric Standard Model (pMSSM) with the SM-like Higgs boson with a mass $\sim 125 \mathrm{GeV}$ as measured by ATLAS and CMS. We performed a parameter scan of the pMSSM with the eight most relevant parameters var- 
ied freely ( $p M S S M$ 8): the $\mathcal{C P}$-odd Higgs boson mass, $M_{A}$, the ratio of the two neutral Higgs vacuum expectation values, $\tan \beta$, a common soft SUSY-breaking parameter for the scalar top- and bottom quarks, $M_{\tilde{q}_{3}}$, a soft SUSY-breaking parameter for the scalar tau and neutrino sector, $M_{\tilde{\ell}_{3}}$, and similarly for the first and second generation of sleptons, $M_{\tilde{\ell}_{1,2}}$, a common trilinear coupling for the third generation, $A_{f}$, the Higgsino mass parameter, $\mu$, as well as the SU(2) gaugino mass parameter, $M_{2}$. The U(1) gaugino mass parameter $M_{1}$ was fixed from the value of $M_{2}$ using the GUT relation. The other parameters have been set to fixed values that are generically in agreement with recent SUSY searches at the LHC.

A random parameter scan with $\mathcal{O}\left(10^{7}\right)$ scan points has been performed. For each scan point a $\chi^{2}$ function was evaluated, taking into account the combined Higgs boson mass measurement of the LHC experiments and the measured rates in 85 individual Higgs search channels from ATLAS, CMS, and the Tevatron experiments (via the code HiggsSignals), the exclusion bounds from the search for additional Higgs bosons (via the code HiggsBound,s, both relying on the evaluations done by FeynHiggs), exclusion bounds from the direct search for SUSY particles (via the code CheckMate), as well as the following low-energy observables $\mathrm{BR}\left(B \rightarrow X_{s} \gamma\right), \mathrm{BR}\left(B_{s} \rightarrow \mu^{+} \mu^{-}\right), \mathrm{BR}\left(B_{u} \rightarrow\right.$ $\left.\tau \nu_{\tau}\right),(g-2)_{\mu}$ (as obtained from Super Iso) and $M_{W}$ (via the prediction of Refs. [132,135]).

Taking into account only the Higgs measurements and direct searches, we find that the SM, the MSSM scenario where the light $\mathcal{C} \mathcal{P}$-even Higgs boson corresponds to the observed signal ("light Higgs case"), as as well as the MSSM scenario where the heavy $\mathcal{C} \mathcal{P}$-even Higgs boson corresponds to the observed signal ("heavy Higgs case") provide similarly good fits to the data with a $\chi^{2} /$ d.o.f. of $70.2 / 86,67.9 / 79$ and 70.0/80, respectively. In a naive evaluation of $p$ values that neglects the correlations between different Higgs observables, this translates into $p$ values of $89 \%, 81 \%$ and $78 \%$, respectively. Including also the low-energy observables we find (via the same evaluation) $p$ values of $69 \%, 89 \%$ and $80 \%$, where the SM suffers in particular from the inclusion of $(g-2)_{\mu}$. Thus, the "light Higgs case" of the MSSM and even the rather exotic "heavy Higgs case" of the MSSM provide a slightly better descriptions of the data in a global fit than the SM.

Within the MSSM, a SM-like Higgs boson at $\sim 125 \mathrm{GeV}$ can be realized in three ways and with similarly good fit qualities. For $M_{A} \gg M_{Z}$ the light $\mathcal{C} \mathcal{P}$-even Higgs boson is SM-like in the decoupling limit. For $M_{A} \lesssim 350 \mathrm{GeV}$ (the "low- $M_{A}$ case") the light $\mathcal{C P}$-even Higgs boson can be SM-like in the limit of alignment without decoupling. This limit also offers additionally the unique possibility that the heavy $\mathcal{C P}$-even Higgs boson can have a mass of $\sim 125 \mathrm{GeV}$ with SM-like couplings. We have analyzed analytically in which parts of the MSSM parameter space the limit of align- ment without decoupling can be realized. In this latter scenario all MSSM Higgs bosons are relatively light, offering good prospects for the searches for additional Higgs bosons at the LHC and future colliders. Our analytic expressions contain the leading two-loop contributions of $\mathcal{O}\left(\alpha_{s} h_{t}^{2}\right)$ [47], which somewhat modify the leading one-loop contributions of $\mathcal{O}\left(h_{t}^{2}\right)$ that had been considered previously [33].

For the light Higgs case in the decoupling limit and in the limit of alignment without decoupling, as well as in the heavy Higgs case we have analyzed the predictions for the various Higgs boson production and decay rates. In the light Higgs case the various rates are predicted to be close to the SM Higgs boson rates, where the largest allowed deviation to smaller values is found in the $h \rightarrow \tau^{+} \tau^{-}$channel. The light scalar top is found to have masses down to $\sim 300 \mathrm{GeV}$, while the preferred region in the fit provides no upper limit on the scalar top quarks. For the low- $M_{A}$ case we find lighter stop masses down to $\sim 500 \mathrm{GeV}$ and $X_{t} / M_{S} \sim+2$. While in the decoupling limit the parameters $\mu$ and $A_{t}$ can vary from very small to very large values, the low- $M_{A}$ case (and thus the limit of alignment without decoupling in the light Higgs interpretation) can be realized only for $\mu / M_{S} \sim 1.4$ to 3 and $A_{t} / M_{S} \sim 2.4$ to 3 , where the upper values correspond to the upper scan ranges imposed in our study. For larger $M_{A}$ values also relatively large values of $\tan \beta$ are still allowed, leading to a sizable contribution to $(g-2)_{\mu}$ even for relatively large chargino/neutralino and slepton masses. Concerning the impact of the low-energy observable, it is interesting to note that a clear preference for positive $\mu$ (and also positive $A_{t}$ ) already emerges when combining the Higgs- and $B$-physics observables in the fit, i.e. already before including $(g-2)_{\mu}$. We have furthermore found that the preferred region in the fit without taking into account the low-energy observables includes predictions for $\operatorname{BR}\left(B_{s} \rightarrow \mu^{+} \mu^{-}\right)$that are close to the experimental central value (i.e., below the SM prediction) both for the decoupling and the alignment without decoupling region of the light Higgs case.

We included the limits from direct LHC searches for SUSY particles from the $8 \mathrm{TeV}$ run via CheckMATE. These searches constrain the parameter space of the pMSSM 8 in an orthogonal way to the Higgs mass and signal rate constraints and therefore do not directly alter the Higgs phenomenology, neither in the light nor the heavy Higgs case. Furthermore, due to this orthogonality we also expect that future stronger SUSY limits from the $13 \mathrm{TeV}$ run would not substantially alter the conclusions found in this paper.

In the heavy Higgs case the preferred rates are also SMlike, however with a possibly larger suppression of $H \rightarrow \gamma \gamma$ and/or $H \rightarrow \tau^{+} \tau^{-}$. We find that in the heavy Higgs case the $\mathcal{C P}$-odd Higgs boson $A$ is restricted to have a mass of $140 \mathrm{GeV} \lesssim M_{A} \lesssim 185 \mathrm{GeV}$, with the charged Higgs boson being the heaviest Higgs boson with $M_{H^{ \pm}} \lesssim 210 \mathrm{GeV}$, and $6 \lesssim \tan \beta \lesssim 11$. We furthermore find $\mu / M_{S} \sim 6$ 
to 9 and $-1 \lesssim A_{t} / M_{S} \lesssim 0$. The light scalar top is predicted to have a mass of $350 \mathrm{GeV} \lesssim m_{\tilde{t}_{1}} \lesssim 650 \mathrm{GeV}$. Due to the relatively small $\tan \beta$ values the scalar leptons have to be relatively light with masses below $\sim 450 \mathrm{GeV}$ to bring the prediction into agreement with the observed discrepancy of the experimental measurement of $(g-2)_{\mu}$ with the SM prediction. In particular, we have checked that the charged Higgs corrections to the $B$-physics observables, $B \rightarrow X_{s} \gamma, B_{u} \rightarrow \tau \nu_{\tau}$ and $B_{s} \rightarrow \mu^{+} \mu^{-}$, are consistent with the experimental results. The preferred region in the fit has mostly light Higgs boson masses above $M_{H} / 2$, in which case $H \rightarrow h h$ decays are kinematically closed. However, also smaller $M_{h}$ values are possible, where $\operatorname{BR}(H \rightarrow h h)$ does not exceed $20 \%$ (for the most favored parameter region we find $\mathrm{BR}(H \rightarrow h h)<2 \%$.) The coupling of the light Higgs boson to $W^{ \pm}$and $Z$ bosons is strongly suppressed, much below the existing bounds from LEP Higgs searches. Note that such a light Higgs boson re-opens the possibility of light neutralino dark matter in the sub-GeV to $65 \mathrm{GeV}$ range by acting as $s$-channel (near-)resonance in dark matter pair-annihilation [174].

As a guidance for the Higgs boson searches in the heavy Higgs interpretation we provide a new set of benchmark scenarios that can be employed to maximize the sensitivity of the experimental analysis to this interpretation. In the $(\mu, \tan \beta)$ plane we define the $l o w-M_{H}^{\text {alt }-}$ and the $l o w-M_{H}^{\text {alt }+}$ scenario with $M_{H^{ \pm}}=155 \mathrm{GeV}<m_{t}$ and $M_{H^{ \pm}}=185 \mathrm{GeV}>$ $m_{t}$, respectively. In the $\left(M_{H^{ \pm}}, \tan \beta\right)$ plane we define the low $-M_{H}^{\text {alt v }}$ scenario with $\mu=6 \mathrm{TeV}$. We have shown that in all three scenarios a parameter regime exists, where the mass and rates of the heavy $\mathcal{C} \mathcal{P}$-even Higgs boson are in agreement with all available measurements, and which is also not excluded by searches for additional Higgs bosons. In the low $-M_{H}^{\text {alt }+}$ scenario we find a very restricted part of the parameter space in which $M_{h} \sim M_{H} \sim 125 \mathrm{GeV}$, with both $\mathcal{C P}$-even Higgs bosons contributing to the Higgs boson rates. The proposed benchmark scenarios will be of interest for upcoming charged Higgs boson searches and will provide motivation for searches for new charged Higgs signatures such as $H^{ \pm} \rightarrow W^{ \pm} h$, with the light Higgs $h$ decaying into bottom quark or tau lepton pairs, $h \rightarrow b \bar{b}, \tau^{+} \tau^{-}$.

New data from the ATLAS and CMS Higgs measurements and the search for new Higgs boson states are now rapidly emerging in the current run of the LHC. It is critical to improve the precision of the measurements of the properties of the SM-like Higgs boson, while improving the sensitivity of the searches for new Higgs bosons with masses either above or below the observed Higgs boson mass. In particular, these searches will yield new constraints on the parameter space of the MSSM. The observation of the SMlike Higgs boson already implies that the MSSM Higgs sector lies close to the alignment limit. Indeed, the regions of the MSSM parameter space in which the approximate alignment limit is realized provide a description of the data that is as good (or in some cases slightly better) than the SM. In addition to the possibility that the observed Higgs boson corresponds to the lighter $\mathcal{C P}$-even Higgs boson of the MSSM, the more exotic possibility in which the heavier $\mathcal{C} \mathcal{P}$-even Higgs boson is identified as the observed Higgs boson cannot yet be ruled out. Higgs studies at Run 2 of the LHC may prove decisive in determining whether the cracks in the Standard Model facade finally shatter, and whether a supersymmetric interpretation of Higgs phenomena is ultimately viable.

Acknowledgements We thank Thomas Hahn, Ben O'Leary, Stefan Liebler, Sebastian Passehr and Florian Staub for helpful discussions and Daniel Schmeier for help with CheckMATE. We are grateful to Oliver Ricken and Peter Wienemann for their technical support and invaluable help with the computer cluster in Bonn. H. E. H. and T. S. are supported in part by the U.S. Department of Energy Grant Number DE-SC0010107. T. S. greatly acknowledges additional support from the Alexander von Humboldt foundation through a Feodor-Lynen research fellowship. The work of S. H. is supported in part by CICYT (Grant FPA 2013-40715-P) and by the Spanish MICINN's Consolider-Ingenio 2010 Program under Grant MultiDark CSD2009-00064. L. Z. is supported by the Netherlands Organization for Scientific Research (NWO) through a VENI grant. G. W. acknowledges support by the DFG through the SFB 676 "Particles, Strings and the Early Universe". This research was supported in part by the European Commission through the "HiggsTools" Initial Training Network PITN-GA-2012-316704.

Open Access This article is distributed under the terms of the Creative Commons Attribution 4.0 International License (http://creativecomm ons.org/licenses/by/4.0/), which permits unrestricted use, distribution, and reproduction in any medium, provided you give appropriate credit to the original author(s) and the source, provide a link to the Creative Commons license, and indicate if changes were made.

Funded by SCOAP ${ }^{3}$.

\section{Appendix A: How tuned is approximate Higgs alignment without decoupling in the MSSM?}

The precision Higgs data implies that the properties of one of the Higgs bosons in the MSSM Higgs sector (which is to be identified with the observed Higgs boson of mass 125 $\mathrm{GeV}$ ) approximate the predicted properties of the SM Higgs boson. This corresponds to the approximate alignment limit described in Sect. 2.2. Approximate alignment can be easily achieved in the parameter regime in which all other (nonSM-like) Higgs boson states are significantly heavier than $125 \mathrm{GeV}$. However, it is also possible that a parameter region of the MSSM exists in which approximate alignment without decoupling is satisfied. In this parameter regime, the other non-SM-like Higgs states are not significantly separated in mass from the observed Higgs boson, which provides additional opportunities for the discovery of new scalar states at the LHC. As shown in Sect. 2.2, alignment without decoupling is achieved when the Higgs basis parameter $\left|Z_{6}\right| \ll 1$ and $M_{A} \lesssim 350 \mathrm{GeV}$. However, a skeptical reader might won- 


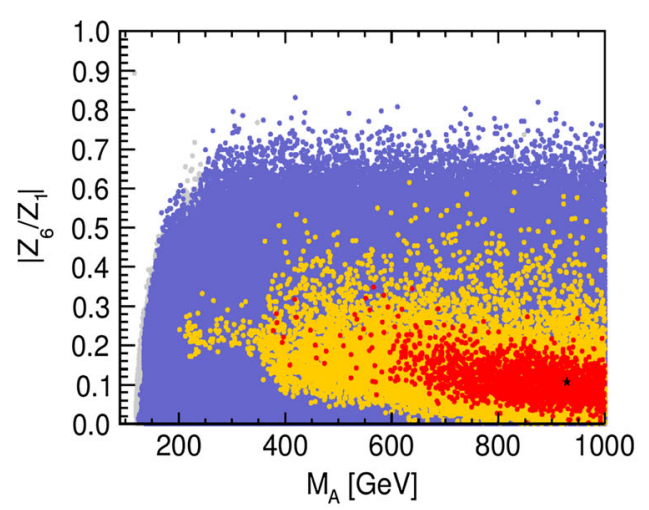

Fig. $28\left|Z_{6} / Z_{1}\right|$ in dependence of the pseudoscalar Higgs mass, $M_{A}$, for the light Higgs (left) and heavy Higgs (right) interpretation. The parameters $Z_{1}$ and $Z_{6}$ are calculated using the approximate two-

der how difficult it is to achieve regions of the MSSM parameter space with very small values of $Z_{6}$. That is, how tuned is approximate Higgs alignment without decoupling in the MSSM?

Consider the case of exact alignment in the two Higgs doublet model (2HDM), which corresponds to $Z_{6}=0$. If exact alignment is a consequence of a global (discrete or continuous) symmetry, then it is natural to consider $2 \mathrm{HDM}$ Higgs sectors that exhibit approximate alignment without decoupling. The possibility of achieving exact alignment by a symmetry of the scalar potential was considered in Refs. $[56,175]$. However, it is not clear whether these symmetries survive once a realistic Higgs-fermion Yukawa sector is considered [176]. The one known example of a realistic 2HDM with exact alignment is the inert doublet model [177], where $Z_{6}=0$ is a consequence of an exact $\mathbb{Z}_{2}$ discrete symmetry, under which the inert doublet field is odd and all other fields are even.

In the MSSM, there is no symmetry associated with the parameter regime corresponding to exact alignment. Indeed, as explained in Sect. 2.2, exact alignment in the MSSM is a result of an accidental cancellation between tree-level and radiatively corrected loop effects that contribute to the effective $Z_{6}$ parameter. In an approximate one-loop expression, this cancellation can be explicitly seen in Eq. (20), where the expression inside the braces vanishes for a particular choice of $\tan \beta$. Of course, given the limited statistics of the present day Higgs data, a region of the MSSM space that exhibits approximate alignment without decoupling can still be consistent with all known experimental constraints. Thus, in any comprehensive scan of the MSSM parameter space, one must necessarily encounter regions of approximate alignment without decoupling. In the absence of a fundamental underlying theory of supersymmetry breaking, the correct measure that governs the MSSM parameter space is unknown. Thus, in order to get a sense of the extent of the tuning associated with the parameter regime of alignment

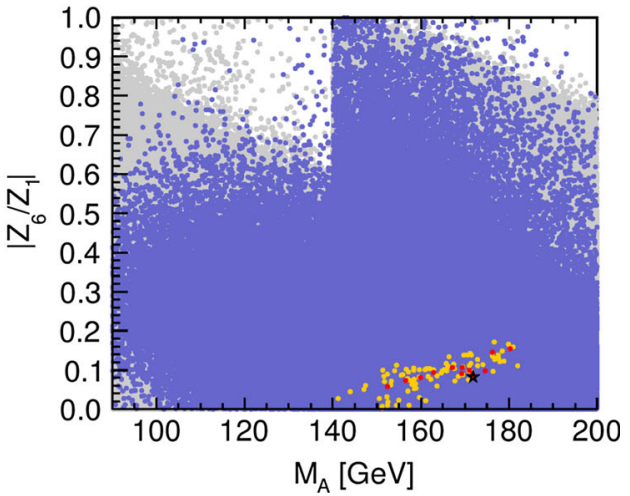

loop formulas as described in Sect. 2.2. The value of $Z_{1}$ is fixed by $M_{h / H} \sim 125 \mathrm{GeV}$ (see text). The color coding is the same as in Fig. 5

without decoupling, the best one can do is to examine the frequency that such parameter points occur in a comprehensive parameter scan (with uniform priors).

In this appendix, we address this last point from a practical point of view. We examine the values of $Z_{6}$ as a function of $M_{A}$ that arise in our pMSSM 8 parameter scans. Note that in the approximate alignment limit, $m_{h}^{2} \simeq Z_{1} v^{2}=$ $(125 \mathrm{GeV})^{2}$, i.e., $Z_{1} \simeq 0.26$, which sets the "natural" size for the other Higgs basis quartic self-couplings (including $Z_{6}$ ). In Fig. 28 we show $\left|Z_{6} / Z_{1}\right|$ as a function of $M_{A}$ for the light Higgs (left) and heavy Higgs (right) interpretation. The parameters $Z_{1}$ and $Z_{6}$ are calculated using the approximate two-loop formulas as described in Sect. 2.2. In the case where $h$ is SM-like, one can see that $\left|Z_{6} / Z_{1}\right| \sim 0.2$ in the region of approximate alignment without decoupling. That is, $Z_{6}$ is suppressed by less than one order of magnitude relative to $Z_{1} \cdot{ }^{24}$ In the case where $H$ is SM-like, values between 0 and 0.2 are found for $\left|Z_{6} / Z_{1}\right|$, with the best-fit value of $\left|Z_{6} / Z_{1}\right| \simeq 0.1$. In both the light and heavy Higgs case, the values of $\left|Z_{6}\right|$ in the regions of approximate alignment without decoupling are not unnaturally small. Indeed, even in the decoupling regime of large $M_{A}$, the typical values of $\left|Z_{6}\right|$ in the preferred MSSM parameter regime are not significantly different in magnitude. We conclude that given the present precision of the Higgs data, approximate alignment without decoupling can be achieved without resorting to an excessive fine tuning of the MSSM parameters.

\section{Appendix B: Higgs measurements from Tevatron and LHC}

Tables 7 and 8 list the 85 signal strength measurements from ATLAS, CMS and the Tevatron (DØ and CDF), which we

\footnotetext{
${ }^{24}$ Indeed, in the case where $h$ is SM-like, regions of exact alignment are ruled out, as these regions correspond to values of $\tan \beta$ that are excluded by the LHC searches for $H, A \rightarrow \tau^{+} \tau^{-}[33,143,178]$.
} 
Table 7 Higgs signal strengths measurements from the LHC ATLAS and Tevatron CDF collaboration

\begin{tabular}{|c|c|c|c|c|c|c|c|}
\hline \multirow[t]{2}{*}{ Analysis } & \multirow{2}{*}{$\begin{array}{l}\text { Energy } \sqrt{s} \\
(\mathrm{TeV})\end{array}$} & \multirow[t]{2}{*}{$\hat{\mu} \pm \Delta \hat{\mu}$} & \multicolumn{5}{|c|}{ SM signal contamination (in \%) } \\
\hline & & & $\operatorname{ggH}$ & VBF & WH & $\mathrm{ZH}$ & $t \bar{t} H$ \\
\hline ATLAS $h \rightarrow W W \rightarrow \ell v \ell v(\mathrm{VBF})[179]$ & $7 / 8$ & $1.27_{-0.45}^{+0.53}$ & 24.1 & 75.9 & 0.0 & 0.0 & 0.0 \\
\hline ATLAS $h \rightarrow W W \rightarrow \ell v \ell v(\mathrm{ggH})$ [179] & $7 / 8$ & $1.01_{-0.25}^{+0.27}$ & 97.8 & 1.2 & 0.6 & 0.3 & 0.1 \\
\hline ATLAS $h \rightarrow Z Z \rightarrow 4 \ell(\mathrm{VBF} / \mathrm{VH})[180]$ & $7 / 8$ & $0.26_{-0.94}^{+1.64}$ & 37.8 & 35.7 & 16.8 & 9.7 & 0.0 \\
\hline ATLAS $h \rightarrow Z Z \rightarrow 4 \ell(\mathrm{ggH})[180]$ & $7 / 8$ & $1.66_{-0.44}^{+0.51}$ & 91.6 & 4.6 & 2.2 & 1.3 & 0.4 \\
\hline ATLAS $h \rightarrow \gamma \gamma(\mathrm{VBF}$, loose) [181] & $7 / 8$ & $1.33_{-0.77}^{+0.92}$ & 39.0 & 60.0 & 0.6 & 0.3 & 0.1 \\
\hline ATLAS $h \rightarrow \gamma \gamma(\mathrm{VBF}$, tight $)[181]$ & $7 / 8$ & $0.68_{-0.51}^{+0.67}$ & 18.2 & 81.5 & 0.1 & 0.1 & 0.1 \\
\hline ATLAS $h \rightarrow \gamma \gamma\left(V h, E_{T}^{\text {miss }}\right)[181]$ & $7 / 8$ & $3.51_{-2.42}^{+3.30}$ & 8.7 & 3.7 & 35.8 & 44.8 & 7.1 \\
\hline ATLAS $h \rightarrow \gamma \gamma(V h, 2 j)[181]$ & $7 / 8$ & $0.23_{-1.39}^{+1.67}$ & 45.0 & 3.3 & 31.9 & 19.8 & 0.1 \\
\hline ATLAS $h \rightarrow \gamma \gamma(V h, 1 \ell)[181]$ & $7 / 8$ & $0.41_{-1.06}^{+1.43}$ & 0.7 & 0.2 & 91.4 & 5.9 & 1.8 \\
\hline ATLAS $h \rightarrow \gamma \gamma\left(\right.$ central, high $\left.p_{T t}\right)$ [181] & $7 / 8$ & $1.62_{-0.83}^{+1.00}$ & 72.6 & 16.4 & 6.1 & 3.7 & 1.2 \\
\hline ATLAS $h \rightarrow \gamma \gamma\left(\right.$ central, low $\left.p_{T t}\right)$ [181] & $7 / 8$ & $0.62_{-0.40}^{+0.42}$ & 93.2 & 4.1 & 1.6 & 1.0 & 0.1 \\
\hline ATLAS $h \rightarrow \gamma \gamma$ (forward, high $p_{T t}$ ) [181] & $7 / 8$ & $1.73_{-1.18}^{+1.34}$ & 71.4 & 16.7 & 6.9 & 4.1 & 0.9 \\
\hline ATLAS $h \rightarrow \gamma \gamma$ (forward, low $p_{T t}$ ) [181] & $7 / 8$ & $2.03_{-0.53}^{+0.57}$ & 92.5 & 4.2 & 2.0 & 1.2 & 0.1 \\
\hline ATLAS $h \rightarrow \gamma \gamma(t t h$, hadr. $)[181]$ & $7 / 8$ & $-0.84_{-1.25}^{+3.23}$ & 15.0 & 1.3 & 1.3 & 1.4 & 81.0 \\
\hline ATLAS $h \rightarrow \gamma \gamma(t t h$, lep. $)[181]$ & $7 / 8$ & $2.42_{-2.07}^{+3.21}$ & 8.4 & 0.1 & 14.9 & 4.0 & 72.6 \\
\hline ATLAS $h \rightarrow \tau \tau$ (VBF, hadr.hadr.) [182] & $7 / 8$ & $1.40_{-0.70}^{+0.90}$ & 30.1 & 69.9 & 0.0 & 0.0 & 0.0 \\
\hline ATLAS $h \rightarrow \tau \tau$ (boosted, hadr.hadr.) [182] & $7 / 8$ & $3.60_{-1.60}^{+2.00}$ & 69.5 & 13.3 & 11.3 & 5.8 & 0.0 \\
\hline ATLAS $h \rightarrow \tau \tau$ (VBF, lep.hadr.) [182] & $7 / 8$ & $1.00_{-0.50}^{+0.60}$ & 17.2 & 82.8 & 0.0 & 0.0 & 0.0 \\
\hline ATLAS $h \rightarrow \tau \tau$ (boosted, lep.hadr.) [182] & $7 / 8$ & $0.90_{-0.90}^{+1.00}$ & 73.0 & 13.3 & 9.1 & 4.6 & 0.0 \\
\hline ATLAS $h \rightarrow \tau \tau$ (VBF, lep.lep.) [182] & $7 / 8$ & $1.80_{-0.90}^{+1.10}$ & 15.4 & 84.6 & 0.0 & 0.0 & 0.0 \\
\hline ATLAS $h \rightarrow \tau \tau$ (boosted, lep.lep.) [182] & $7 / 8$ & $3.00_{-1.70}^{+1.90}$ & 70.9 & 21.4 & 5.7 & 2.1 & 0.0 \\
\hline ATLAS $V h \rightarrow V(b b)(0 \ell)[183]$ & $7 / 8$ & $-0.35_{-0.52}^{+0.55}$ & 0.0 & 0.0 & 20.8 & 79.2 & 0.0 \\
\hline ATLAS $V h \rightarrow V(b b)(1 \ell)[183]$ & $7 / 8$ & $1.17_{-0.60}^{+0.66}$ & 0.0 & 0.0 & 96.7 & 3.3 & 0.0 \\
\hline ATLAS $V h \rightarrow V(b b)(2 \ell)[183]$ & $7 / 8$ & $0.94_{-0.79}^{+0.88}$ & 0.0 & 0.0 & 0.0 & 100.0 & 0.0 \\
\hline ATLAS $V h \rightarrow V(W W)(2 \ell)[184]$ & $7 / 8$ & $3.70_{-1.80}^{+1.90}$ & 0.0 & 0.0 & 83.3 & 16.7 & 0.0 \\
\hline ATLAS $V h \rightarrow V(W W)(3 \ell)[184]$ & $7 / 8$ & $0.72_{-1.10}^{+1.30}$ & 0.0 & 0.0 & 86.5 & 13.5 & 0.0 \\
\hline ATLAS $V h \rightarrow V(W W)(4 \ell)[184]$ & $7 / 8$ & $4.90_{-3.10}^{+4.60}$ & 0.0 & 0.0 & 0.0 & 100.0 & 0.0 \\
\hline ATLAS $t t h \rightarrow$ multilepton $\left(1 \ell, 2 \tau_{h}\right)[185]$ & $7 / 8$ & $-9.60_{-9.70}^{+9.60}$ & 0.0 & 0.0 & 0.0 & 0.0 & $100.0^{1}$ \\
\hline ATLAS $t t h \rightarrow$ multilepton $\left(2 \ell, 0 \tau_{h}\right)[185]$ & $7 / 8$ & $2.80_{-1.90}^{+2.10}$ & 0.0 & 0.0 & 0.0 & 0.0 & $100.0^{2}$ \\
\hline ATLAS $t t h \rightarrow$ multilepton $\left(2 \ell, 1 \tau_{h}\right)[185]$ & $7 / 8$ & $-0.90_{-2.00}^{+3.10}$ & 0.0 & 0.0 & 0.0 & 0.0 & $100.0^{3}$ \\
\hline ATLAS $t t h \rightarrow$ multilepton $(3 \ell)[185]$ & $7 / 8$ & $2.80_{-1.80}^{+2.20}$ & 0.0 & 0.0 & 0.0 & 0.0 & $100.0^{4}$ \\
\hline ATLAS $t t h \rightarrow$ multilepton $(4 \ell)$ [185] & $7 / 8$ & $1.80_{-6.90}^{+6.90}$ & 0.0 & 0.0 & 0.0 & 0.0 & $100.0^{5}$ \\
\hline ATLAS $t t h \rightarrow t t(b b)[186]$ & $7 / 8$ & $1.50_{-1.10}^{+1.10}$ & 0.0 & 0.0 & 0.0 & 0.0 & 100.0 \\
\hline $\mathrm{CDF} h \rightarrow W W[187]$ & 1.96 & $0.00_{-1.78}^{+1.78}$ & 77.5 & 5.4 & 10.6 & 6.5 & 0.0 \\
\hline $\mathrm{CDF} h \rightarrow \gamma \gamma[187]$ & 1.96 & $7.81_{-4.42}^{+4.61}$ & 77.5 & 5.4 & 10.6 & 6.5 & 0.0 \\
\hline $\mathrm{CDF} h \rightarrow \tau \tau[187]$ & 1.96 & $0.00_{-8.44}^{+8.44}$ & 77.5 & 5.4 & 10.6 & 6.5 & 0.0 \\
\hline $\mathrm{CDF} V h \rightarrow V(b b)[187]$ & 1.96 & $1.72_{-0.87}^{+0.92}$ & 0.0 & 0.0 & 62.0 & 38.0 & 0.0 \\
\hline $\mathrm{CDF} t t h \rightarrow t t(b b)[187]$ & 1.96 & $9.49_{-6.28}^{+6.60}$ & 0.0 & 0.0 & 0.0 & 0.0 & 100.0 \\
\hline
\end{tabular}

${ }^{1}$ The SM Higgs signal composition is $h \rightarrow \tau \tau(93.0 \%), h \rightarrow W W(4.0 \%), h \rightarrow b b(3.0 \%)$

2 The SM Higgs signal composition is $h \rightarrow W W(80.1 \%), h \rightarrow \tau \tau(14.9 \%), h \rightarrow Z Z(3.0 \%), h \rightarrow b b(2.0 \%)$

${ }^{3}$ The SM Higgs signal composition is $h \rightarrow \tau \tau$ (61.8\%), $h \rightarrow W W(35.2 \%), h \rightarrow Z Z(2.0 \%), h \rightarrow b b(1.0 \%)$

${ }^{4}$ The SM Higgs signal composition is $h \rightarrow W W(74.1 \%), h \rightarrow \tau \tau(14.9 \%), h \rightarrow Z Z(7.0 \%), h \rightarrow b b(3.9 \%)$

${ }^{5}$ The SM Higgs signal composition is $h \rightarrow W W(68.1 \%), h \rightarrow \tau \tau(13.9 \%), h \rightarrow Z Z(14.0 \%), h \rightarrow b b(4.0 \%)$ 
Table 8 Higgs signal strengths measurements from LHC CMS and Tevatron D $\emptyset$ collaboration

\begin{tabular}{|c|c|c|c|c|c|c|c|}
\hline \multirow[t]{2}{*}{ Analysis } & \multirow{2}{*}{$\begin{array}{l}\text { Energy } \sqrt{s} \\
(\mathrm{TeV})\end{array}$} & \multirow[t]{2}{*}{$\hat{\mu} \pm \Delta \hat{\mu}$} & \multicolumn{5}{|c|}{ SM signal contamination (in \%) } \\
\hline & & & $\mathrm{ggH}$ & VBF & WH & $\mathrm{ZH}$ & $t \bar{t} H$ \\
\hline $\mathrm{CMS} h \rightarrow W W \rightarrow 2 \ell 2 v(0 / 1 j)[188]$ & $7 / 8$ & $0.74_{-0.20}^{+0.22}$ & 85.8 & 8.9 & 3.3 & 1.9 & 0.0 \\
\hline CMS $h \rightarrow W W \rightarrow 2 \ell 2 v$ (VBF) [188] & $7 / 8$ & $0.60_{-0.46}^{+0.57}$ & 24.1 & 75.9 & 0.0 & 0.0 & 0.0 \\
\hline $\mathrm{CMS} h \rightarrow Z Z \rightarrow 4 \ell(0 / 1 j)[189]$ & $7 / 8$ & $0.88_{-0.27}^{+0.34}$ & 91.9 & 8.1 & 0.0 & 0.0 & 0.0 \\
\hline CMS $h \rightarrow Z Z \rightarrow 4 \ell(2 j)$ [189] & $7 / 8$ & $1.55_{-0.66}^{+0.95}$ & 76.1 & 23.9 & 0.0 & 0.0 & 0.0 \\
\hline CMS $h \rightarrow \gamma \gamma$ (untagged 0) [190] & 7 & $1.97_{-1.25}^{+1.51}$ & 80.8 & 9.7 & 5.8 & 3.2 & 0.6 \\
\hline CMS $h \rightarrow \gamma \gamma$ (untagged 1) [190] & 7 & $1.23_{-0.88}^{+0.98}$ & 92.3 & 4.1 & 2.3 & 1.2 & 0.1 \\
\hline CMS $h \rightarrow \gamma \gamma$ (untagged 2) [190] & 7 & $1.60_{-1.17}^{+1.25}$ & 92.3 & 4.0 & 2.3 & 1.3 & 0.1 \\
\hline CMS $h \rightarrow \gamma \gamma$ (untagged 3) [190] & 7 & $2.61_{-1.65}^{+1.74}$ & 92.5 & 3.9 & 2.3 & 1.2 & 0.1 \\
\hline $\mathrm{CMS} h \rightarrow \gamma \gamma(\mathrm{VBF}$, dijet 0) [190] & 7 & $4.85_{-1.76}^{+2.17}$ & 19.9 & 79.6 & 0.3 & 0.2 & 0.1 \\
\hline CMS $h \rightarrow \gamma \gamma$ (VBF, dijet 1) [190] & 7 & $2.60_{-1.76}^{+2.16}$ & 39.0 & 58.9 & 1.2 & 0.7 & 0.3 \\
\hline $\mathrm{CMS} h \rightarrow \gamma \gamma\left(V h, E_{T}^{\mathrm{miss}}\right)[190]$ & 7 & $4.32_{-4.15}^{+6.72}$ & 4.9 & 1.2 & 43.2 & 44.4 & 6.3 \\
\hline CMS $h \rightarrow \gamma \gamma(V h$, dijet) [190] & 7 & $7.86_{-6.40}^{+8.86}$ & 28.6 & 2.9 & 43.8 & 23.3 & 1.5 \\
\hline CMS $h \rightarrow \gamma \gamma(V h$, loose $)[190]$ & 7 & $3.10_{-5.34}^{+8.29}$ & 3.8 & 1.1 & 79.7 & 14.6 & 0.7 \\
\hline CMS $h \rightarrow \gamma \gamma(t t h$, tags $)[190]$ & 7 & $0.71_{-3.56}^{+6.20}$ & 4.3 & 1.5 & 2.9 & 1.6 & 89.7 \\
\hline CMS $h \rightarrow \gamma \gamma$ (untagged 0) [190] & 8 & $0.13_{-0.74}^{+1.09}$ & 75.7 & 11.9 & 6.9 & 3.6 & 1.9 \\
\hline CMS $h \rightarrow \gamma \gamma$ (untagged 1) [190] & 8 & $0.92_{-0.49}^{+0.57}$ & 85.1 & 7.9 & 4.0 & 2.4 & 0.6 \\
\hline CMS $h \rightarrow \gamma \gamma$ (untagged 2) [190] & 8 & $1.10_{-0.44}^{+0.48}$ & 91.1 & 4.7 & 2.5 & 1.4 & 0.3 \\
\hline CMS $h \rightarrow \gamma \gamma$ (untagged 3) [190] & 8 & $0.65_{-0.89}^{+0.65}$ & 91.5 & 4.4 & 2.4 & 1.4 & 0.3 \\
\hline CMS $h \rightarrow \gamma \gamma$ (untagged 4) [190] & 8 & $1.46_{-1.24}^{+1.29}$ & 93.1 & 3.6 & 2.0 & 1.1 & 0.2 \\
\hline CMS $h \rightarrow \gamma \gamma$ (VBF, dijet 0) [190] & 8 & $0.82_{-0.58}^{+0.75}$ & 17.8 & 81.8 & 0.2 & 0.1 & 0.1 \\
\hline CMS $h \rightarrow \gamma \gamma$ (VBF, dijet 1) [190] & 8 & $-0.21_{-0.69}^{+0.75}$ & 28.4 & 70.6 & 0.6 & 0.2 & 0.2 \\
\hline CMS $h \rightarrow \gamma \gamma$ (VBF, dijet 2) [190] & 8 & $2.60_{-0.99}^{+1.33}$ & 43.7 & 53.3 & 1.4 & 0.8 & 0.8 \\
\hline $\mathrm{CMS} h \rightarrow \gamma \gamma\left(V h, E_{T}^{\mathrm{miss}}\right)[190]$ & 8 & $0.08_{-1.28}^{+1.86}$ & 16.5 & 2.7 & 34.4 & 35.3 & 11.1 \\
\hline CMS $h \rightarrow \gamma \gamma(V h$, dijet $)[190]$ & 8 & $0.39_{-1.48}^{+2.16}$ & 30.4 & 3.1 & 40.5 & 23.3 & 2.6 \\
\hline CMS $h \rightarrow \gamma \gamma(V h$, loose $)[190]$ & 8 & $1.24_{-2.62}^{+3.69}$ & 2.7 & 1.1 & 77.9 & 16.8 & 1.5 \\
\hline CMS $h \rightarrow \gamma \gamma(V h$, tight $)[190]$ & 8 & $-0.34_{-0.63}^{+1.30}$ & 0.2 & 0.2 & 76.9 & 19.0 & 3.7 \\
\hline CMS $h \rightarrow \gamma \gamma(t t h$, multijet) [190] & 8 & $1.24_{-2.70}^{+4.23}$ & 4.1 & 0.9 & 0.8 & 0.9 & 93.3 \\
\hline CMS $h \rightarrow \gamma \gamma$ (tth, lepton) [190] & 8 & $3.52_{-2.45}^{+3.89}$ & 0.0 & 0.0 & 1.9 & 1.9 & 96.1 \\
\hline $\mathrm{CMS} h \rightarrow \mu \mu[191]$ & $7 / 8$ & $2.90_{-2.70}^{+2.80}$ & 94.1 & 5.9 & 0.0 & 0.0 & 0.0 \\
\hline $\mathrm{CMS} h \rightarrow \tau \tau(0 j)[192]$ & $7 / 8$ & $0.40_{-1.13}^{+0.73}$ & 98.5 & 0.8 & 0.4 & 0.3 & 0.0 \\
\hline $\mathrm{CMS} h \rightarrow \tau \tau(1 j)[192]$ & $7 / 8$ & $1.06_{-0.47}^{+0.47}$ & 79.7 & 12.1 & 5.2 & 3.0 & 0.0 \\
\hline $\mathrm{CMS} h \rightarrow \tau \tau(\mathrm{VBF})[192]$ & $7 / 8$ & $0.93_{-0.41}^{+0.41}$ & 20.9 & 79.1 & 0.0 & 0.0 & 0.0 \\
\hline CMS $V h \rightarrow V(\tau \tau)[192]$ & $7 / 8$ & $0.98_{-1.50}^{+1.68}$ & 0.0 & 0.0 & $47.1^{1}$ & $27.3^{1}$ & 0.0 \\
\hline CMS $V h \rightarrow V(b b)[193]$ & $7 / 8$ & $1.00_{-0.49}^{+0.51}$ & 0.0 & 0.0 & 63.3 & 36.7 & 0.0 \\
\hline CMS $V h \rightarrow V(W W) \rightarrow 2 \ell 2 v[188]$ & $7 / 8$ & $0.39_{-1.87}^{+1.97}$ & 60.2 & 3.8 & 22.8 & 13.2 & 0.0 \\
\hline CMS $V h \rightarrow V(W W)$ (hadr.) [188] & $7 / 8$ & $1.00_{-2.00}^{+2.00}$ & 63.7 & 3.3 & 21.9 & 11.1 & 0.0 \\
\hline CMS $W h \rightarrow W(W W) \rightarrow 3 \ell 3 v[188]$ & $7 / 8$ & $0.56_{-0.95}^{+1.27}$ & 0.0 & 0.0 & 100.0 & 0.0 & 0.0 \\
\hline CMS $t t h \rightarrow 2 \ell$ (same-sign) [194] & $7 / 8$ & $5.30_{-1.80}^{+2.10}$ & 0.0 & 0.0 & 0.0 & 0.0 & $100.0^{2}$ \\
\hline CMS $t t h \rightarrow 3 \ell[194]$ & $7 / 8$ & $3.10_{-2.00}^{+2.40}$ & 0.0 & 0.0 & 0.0 & 0.0 & $100.0^{3}$ \\
\hline CMS $t t h \rightarrow 4 \ell[194]$ & $7 / 8$ & $-4.70_{-1.30}^{+5.00}$ & 0.0 & 0.0 & 0.0 & 0.0 & $100.0^{4}$ \\
\hline $\mathrm{CMS} t t h \rightarrow t t(b b)[194]$ & $7 / 8$ & $0.70_{-1.90}^{+1.90}$ & 0.0 & 0.0 & 0.0 & 0.0 & 100.0 \\
\hline $\mathrm{CMS} t t h \rightarrow t t(\gamma \gamma)[194]$ & $7 / 8$ & $2.70_{-1.80}^{+2.60}$ & 0.0 & 0.0 & 0.0 & 0.0 & 100.0 \\
\hline
\end{tabular}


Table 8 continued

\begin{tabular}{|c|c|c|c|c|c|c|c|}
\hline \multirow[t]{2}{*}{ Analysis } & \multirow{2}{*}{$\begin{array}{l}\text { Energy } \sqrt{s} \\
(\mathrm{TeV})\end{array}$} & \multirow[t]{2}{*}{$\hat{\mu} \pm \Delta \hat{\mu}$} & \multicolumn{5}{|c|}{ SM signal contamination (in \%) } \\
\hline & & & $\mathrm{ggH}$ & VBF & WH & $\mathrm{ZH}$ & $t \bar{t} H$ \\
\hline $\mathrm{CMS} t t h \rightarrow t t(\tau \tau)$ [194] & $7 / 8$ & $-1.30_{-5.50}^{+6.30}$ & 0.0 & 0.0 & 0.0 & 0.0 & 100.0 \\
\hline $\mathrm{D} \varnothing h \rightarrow W W[195]$ & 1.96 & $1.90_{-1.52}^{+1.63}$ & 77.5 & 5.4 & 10.6 & 6.5 & 0.0 \\
\hline $\mathrm{D} \varnothing h \rightarrow b b[195]$ & 1.96 & $1.23_{-1.17}^{+1.24}$ & 0.0 & 0.0 & 62.0 & 38.0 & 0.0 \\
\hline $\mathrm{D} \varnothing h \rightarrow \gamma \gamma[195]$ & 1.96 & $4.20_{-4.20}^{+4.60}$ & 77.5 & 5.4 & 10.6 & 6.5 & 0.0 \\
\hline $\mathrm{D} \varnothing h \rightarrow \tau \tau[195]$ & 1.96 & $3.96_{-3.38}^{+4.11}$ & 77.5 & 5.4 & 10.6 & 6.5 & 0.0 \\
\hline
\end{tabular}

${ }^{1}$ The signal is contaminated to $16.2 \%[9.4 \%]$ by $W H \rightarrow W W W[Z H \rightarrow Z W W]$ in the SM

2 The SM Higgs signal composition is $h \rightarrow W W(73.3 \%), h \rightarrow \tau \tau(23.1 \%), h \rightarrow Z Z(3.6 \%)$

${ }^{3}$ The SM Higgs signal composition is $h \rightarrow W W(71.8 \%), h \rightarrow \tau \tau(23.8 \%), h \rightarrow Z Z(4.4 \%)$

${ }^{4}$ The SM Higgs signal composition is $h \rightarrow W W(53.0 \%), h \rightarrow \tau \tau(30.1 \%), h \rightarrow Z Z(16.9 \%)$

include in our analysis via HiggsSignals-1.4.0. For each analysis, we give the measured signal strength value, $\hat{\mu}$, its $1 \sigma$ uncertainty, $\Delta \hat{\mu}$, as well as the signal composition for the production of a SM Higgs with mass $\sim 125 \mathrm{GeV}$.

\section{References}

1. ATLAS Collaboration, G. Aad et al., Phys. Lett. B 716, 1-29 (2012). arXiv: 1207.7214

2. CMS Collaboration, S. Chatrchyan et al., Phys. Lett. B 716, 30-61 (2012). arXiv:1207.7235

3. ATLAS, CMS Collaborations, G. Aad et al., Phys. Rev. Lett. 114, 191803 (2015). arXiv:1503.07589

4. H.P. Nilles, Phys. Rep. 110, 1-162 (1984)

5. H.E. Haber, G.L. Kane, Phys. Rep. 117, 75-263 (1985)

6. R. Barbieri, Riv. Nuovo Cim. 11N4, 1-45 (1988)

7. S. Heinemeyer, O. Stål, G. Weiglein, Phys. Lett. B 710, 201-206 (2014). arXiv:1112.3026

8. A. Pilaftsis, Phys. Rev. D 58, 096010 (1998). arXiv:hep-ph/ 9803297

9. A. Pilaftsis, Phys. Lett. B 435, 88-100 (1998). arXiv:hep-ph/ 9805373

10. D.A. Demir, Phys. Rev. D 60, 055006 (1999). arXiv:hep-ph/ 9901389

11. A. Pilaftsis, C.E.M. Wagner, Nucl. Phys. B 553, 3-42 (1999). arXiv:hep-ph/9902371

12. S. Heinemeyer, Eur. Phys. J. C 22, 521-534 (2001). arXiv:hep-ph/ 0108059

13. ALEPH, DELPHI, L3 and OPAL Collaborations, S. Schael et al., Eur. Phys. J. C 47, 547-587 (2006). arXiv:hep-ex/0602042

14. A. Djouadi, Phys. Rept. 459, 1-241 (2008). arXiv:hep-ph/ 0503173

15. S. Heinemeyer, Int. J. Mod. Phys. A 21, 2659-2772 (2006). arXiv:hep-ph/0407244

16. S. Heinemeyer, W. Hollik, G. Weiglein, Phys. Rep. 425, 265-368 (2006). arXiv:hep-ph/0412214

17. P. Bechtle, S. Heinemeyer, O. Stål, T. Stefaniak, G. Weiglein, JHEP 11, 039 (2014). arXiv:1403.1582

18. C. Englert, A. Freitas, M.M. Mühlleitner, T. Plehn, M. Rauch, M. Spira, J. Phys. G 41, 113001 (2014). arXiv:1403.7191

19. P. Bechtle, S. Heinemeyer, O. Stål, T. Stefaniak, G. Weiglein, L. Zeune, Eur. Phys. J. C 73(4), 2354 (2013). arXiv:1211.1955

20. S. Scopel, N. Fornengo, A. Bottino, Phys. Rev. D 88, 023506 (2013). arXiv:1304.5353
21. A. Djouadi, Eur. Phys. J. C 74, 2704 (2014). arXiv:1311.0720

22. K. Cheung, J.S. Lee, P.-Y. Tseng, Phys. Rev. D 92, 095004 (2015). arXiv:1501.03552

23. P. Bechtle, S. Heinemeyer, O. Stål, T. Stefaniak, G. Weiglein, Eur. Phys. J. C 75(9), 421 (2015). arXiv: 1507.06706

24. B. Bhattacherjee, A. Chakraborty, A. Choudhury, Phys. Rev. D 92(9), 093007 (2015). arXiv:1504.04308

25. K.J. de Vries et al., Eur. Phys. J. C 75(9), 422 (2015) arXiv: 1504.03260

26. S. Henrot-Versillé, R. Lafaye, T. Plehn, M. Rauch, D. Zerwas, S. Plaszczynski, B. Rouillé d'Orfeuil, M. Spinelli, Phys. Rev. D 89(5), 055017 (2014). arXiv:1309.6958

27. G. Bertone, F. Calore, S. Caron, R. Ruiz, J.S. Kim, R. Trotta, C. Weniger, JCAP 1604(04), 037 (2016). arXiv: 1507.07008

28. H.E. Haber, Y. Nir, Nucl. Phys. B 335, 363-394 (1990)

29. H. E. Haber in Perspectives for Electroweak Interactions in e+ e-Collisions. Proceedings, Ringberg Workshop, Tegernsee, Germany, February 5-8, 1995 (1995). arXiv:hep-ph/9505240

30. A. Dobado, M.J. Herrero, S. Penaranda, Eur. Phys. J. C 17, 487500 (2000). arXiv:hep-ph/0002134

31. J.F. Gunion, H.E. Haber, Phys. Rev. D 67, 075019 (2003). arXiv:hep-ph/0207010

32. M. Carena, I. Low, N.R. Shah, C.E.M. Wagner, JHEP 04, 015 (2014). arXiv: 1310.2248

33. M. Carena, H.E. Haber, I. Low, N.R. Shah, C.E.M. Wagner, Phys. Rev. D 91(3), 035003 (2015). arXiv: 1410.4969

34. K. Hagiwara, J.S. Lee, J. Nakamura, JHEP 10, 002 (2012). arXiv: 1207.0802

35. R. Benbrik, M. Gomez Bock, S. Heinemeyer, O. Stål, G. Weiglein, Eur. Phys. J. C 72, 2171 (2012). arXiv:1207.1096

36. M. Drees, Phys. Rev. D 86, 115018 (2012). arXiv:1210.6507

37. T. Han, T. Li, S. Su, L.-T. Wang, JHEP 11, 053 (2013). arXiv: 1306.3229

38. J.-J. Cao, Z.-X. Heng, J.M. Yang, Y.-M. Zhang, J.-Y. Zhu, JHEP 03, 086 (2012). arXiv:1202.5821

39. S.F. King, M. Muhlleitner, R. Nevzorov, K. Walz, Phys. Rev. D 90(9), 095014 (2014). arXiv:1408.1120

40. F. Domingo, G. Weiglein, JHEP 04, 095 (2016), arXiv:1509. 07283

41. U. Ellwanger, M. Rodriguez-Vazquez, JHEP 02, 096 (2016). arXiv: 1512.04281

42. P. Drechsel, L. Galeta, S. Heinemeyer, and G. Weiglein arXiv: 1601.08100

43. M. Carena, S. Heinemeyer, O. Stål, C.E.M. Wagner, G. Weiglein, Eur. Phys. J. C 73(9), 2552 (2013). arXiv:1302.7033 
44. ATLAS Collaboration, G. Aad et al., JHEP 03, 088 (2015). arXiv: 1412.6663

45. CMS Collaboration, V. Khachatryan et al., JHEP 11, 018 (2015). arXiv: 1508.07774

46. ATLAS, CMS Collaborations, G. Aad et al., JHEP 08, 045 (2016). arXiv: 1606.02266

47. P. Bechtle, H. Haber, S. Heinemeyer, T. Stefaniak, G. Weiglein, L. Zeune (in preparation)

48. H. Georgi, D.V. Nanopoulos, Phys. Lett. B 82, 95-96 (1979)

49. G.C. Branco, L. Lavoura, J.P. Silva, CP Violation (Oxford University Press, Oxford, 1999)

50. S. Davidson, H.E. Haber, Phys. Rev. D 72, 035004 (2005). arXiv:hep-ph/0504050 [Erratum: Phys. Rev. D 72, 099902 (2005)]

51. J. Bernon, J.F. Gunion, H.E. Haber, Y. Jiang, S. Kraml, Phys. Rev. D 92(7), 075004 (2015). arXiv:1507.00933

52. J. Bernon, J.F. Gunion, H.E. Haber, Y. Jiang, S. Kraml, Phys. Rev. D 93(3), 035027 (2016). arXiv: 1511.03682

53. J.F. Gunion, H.E. Haber, G.L. Kane, S. Dawson, The Higgs Hunter's Guide (Westview Press, Boulder, 2000)

54. N. Craig, J. Galloway, S. Thomas, arXiv:1305.2424

55. H. E. Haber in 1st Toyama International Workshop on Higgs as a Probe of New Physics 2013 (HPNP2013) Toyama, Japan, February 13-16, 2013 (2013). arXiv:1401.0152

56. P.S. Bhupal Dev, A. Pilaftsis, JHEP 12, 024 (2014). arXiv:1408.3405 [Erratum: JHEP 11, 147 (2015)]

57. M. Carena, S. Heinemeyer, O. Stål, C. Wagner, G. Weiglein, Eur. Phys. J. C 73(9), 2552 (2013). arXiv:1302.7033

58. H.E. Haber, R. Hempfling, Phys. Rev. D 48, 4280-4309 (1993). arXiv:hep-ph/9307201

59. M. Carena, H.E. Haber, S. Heinemeyer, W. Hollik, C.E.M. Wagner, G. Weiglein, Nucl. Phys. B 580, 29-57 (2000). arXiv:hep-ph/0001002

60. D. Buttazzo, G. Degrassi, P.P. Giardino, G.F. Giudice, F. Sala, A. Salvio, A. Strumia, JHEP 12, 089 (2013). arXiv:1307.3536

61. G. Degrassi, S. Heinemeyer, W. Hollik, P. Slavich, G. Weiglein, Eur. Phys. J. C 28, 133-143 (2003). arXiv:hep-ph/0212020

62. H.E. Haber, J.D. Mason, Phys. Rev. D 77, 115011 (2008). arXiv:0711.2890

63. A. Crivellin, Phys. Rev. D 83, 056001 (2011). arXiv: 1012.4840

64. M. Carena, H.E. Haber, Prog. Part. Nucl. Phys. 50, 63-152 (2003). arXiv:hep-ph/0208209

65. M. Dine, N. Seiberg, S. Thomas, Phys. Rev. D 76, 095004 (2007). arXiv:0707.0005

66. L.J. Hall, M.B. Wise, Nucl. Phys. B 187, 397-408 (1981)

67. R. Hempfling, Phys. Rev. D 49, 6168-6172 (1994)

68. L.J. Hall, R. Rattazzi, U. Sarid, Phys. Rev. D 50, 7048-7065 (1994). arXiv:hep-ph/9306309

69. M. Carena, M. Olechowski, S. Pokorski, C. Wagner, Nucl. Phys. B 426, 269-300 (1994). arXiv:hep-ph/9402253

70. A. Bartl, H. Eberl, K. Hidaka, T. Kon, W. Majerotto, Y. Yamada, Phys. Lett. B 378, 167-174 (1996). arXiv:hep-ph/9511385

71. J.A. Coarasa Perez, R.A. Jimenez, J. Sola, Phys. Lett. B389, 312 320 (1996). arXiv:hep-ph/9511402

72. R.A. Jimenez, J. Sola, Phys. Lett. B 389, 53-61 (1996). arXiv:hep-ph/9511292

73. D.M. Pierce, J.A. Bagger, K.T. Matchev, R.-J. Zhang, Nucl. Phys. B 491, 3-67 (1997). arXiv:hep-ph/9606211

74. M. Carena, D. Garcia, U. Nierste, C.E.M. Wagner, Nucl. Phys. B 577, 88-120 (2000). arXiv:hep-ph/9912516

75. H.E. Haber, M.J. Herrero, H.E. Logan, S. Penaranda, S. Rigolin, D. Temes, Phys. Rev. D 63, 055004 (2001). arXiv:hep-ph/0007006

76. M. Carena, D. Garcia, U. Nierste, C.E.M. Wagner, Phys. Lett. B 499, 141-146 (2001). arXiv:hep-ph/0010003

77. J.R. Ellis, S. Rudaz, Phys. Lett. B 128, 248-252 (1983)
78. J. Frere, D. Jones, S. Raby, Nucl. Phys. B 222, 11 (1983)

79. M. Claudson, L.J. Hall, I. Hinchliffe, Nucl. Phys. B 228, 501 (1983)

80. C. Kounnas, A. Lahanas, D.V. Nanopoulos, M. Quiros, Nucl. Phys. B 236, 438 (1984)

81. J. Gunion, H. Haber, M. Sher, Nucl. Phys. B 306, 1 (1988)

82. J. Casas, A. Lleyda, C. Munoz, Nucl. Phys. B 471, 3-58 (1996). arXiv:hep-ph/9507294

83. P. Langacker, N. Polonsky, Phys. Rev. D 50, 2199-2217 (1994). arXiv:hep-ph/9403306

84. A. Strumia, Nucl. Phys. B 482, 24-38 (1996). arXiv:hep-ph/ 9604417

85. D. Chowdhury, R.M. Godbole, K.A. Mohan, K. Vempati, JHEP 02, 110 (2014). arXiv:1310.1932

86. E. Bagnaschi, F. Brümmer, W. Buchmüller, A. Voigt, G. Weiglein, JHEP 03, 158 (2016). arXiv: 1512.07761

87. W.G. Hollik, JHEP 08, 126 (2016). arXiv:1606.08356

88. ATLAS, CDF, CMS, D0 Collaborations, arXiv:1403.4427

89. http://www.feynhiggs.de

90. S. Heinemeyer, W. Hollik, G. Weiglein, Eur. Phys. J. C 9, 343-366 (1999). arXiv:hep-ph/9812472

91. S. Heinemeyer, W. Hollik, G. Weiglein, Comput. Phys. Commun. 124, 76-89 (2000). arXiv:hep-ph/9812320

92. M. Frank, T. Hahn, S. Heinemeyer, W. Hollik, H. Rzehak et al., JHEP 02, 047 (2007). arXiv:hep-ph/0611326

93. T. Hahn, S. Heinemeyer, W. Hollik, H. Rzehak, G. Weiglein, Comput. Phys. Commun. 180, 1426-1427 (2009)

94. T. Hahn, S. Heinemeyer, W. Hollik, H. Rzehak, G. Weiglein, Phys. Rev. Lett. 112(14), 141801 (2014). arXiv:1312.4937

95. P. Bechtle, S. Heinemeyer, O. Stål, T. Stefaniak, G. Weiglein, Eur. Phys. J. C 74(2), 2711 (2014). arXiv:1305.1933

96. O. Stål, I. Stefaniak, PoS EPS-HEP2013, 314 (2013). arXiv: 1310.4039

97. E. Fuchs, S. Thewes, Eur. Phys. J. C 75, 254 (2015). arXiv: 1411.4652

98. LHC Higgs Cross Section Working Group Collaboration, S. Dittmaier et al., Handbook of LHC Higgs Cross Sections: 1. Inclusive Observables, CERN Yellow Report CERN-2011-002. arXiv: 1101.0593

99. LHC Higgs Cross Section Working Group Collaboration, S. Dittmaier et al., Handbook of LHC Higgs Cross Sections: 2. Differential Distributions, CERN Yellow Report CERN-2012-002. arXiv: 1201.3084

100. https://twiki.cern.ch/twiki/bin/view/LHCPhysics/CrossSections

101. D. de Florian, M. Grazzini, Phys. Lett. B 674, 291-294 (2009). arXiv:0901.2427

102. See http://theory.fi.infn.it/grazzini/hcalculators.html

103. T. Hahn, S. Heinemeyer, F. Maltoni, G. Weiglein, S. Willenbrock, arXiv: hep-ph/0607308

104. T. Hahn, S. Heinemeyer, W. Hollik, H. Rzehak, G. Weiglein, Nucl. Phys. Proc. Suppl. 205-206, 152-157 (2010). arXiv:1007.0956

105. S. Heinemeyer, W. Hollik, G. Weiglein, Eur. Phys. J. C16, 139153 (2000). arXiv:hep-ph/0003022

106. K.E. Williams, H. Rzehak, G. Weiglein, Eur. Phys. J. C 71, 1669 (2011). arXiv:1103.1335

107. BaBar Collaboration, J.P. Lees et al., Phys. Rev. Lett. 109, 101802 (2012). arXiv:1205.5442

108. Belle Collaboration, A. Abdesselam et al., arXiv:1603.06711

109. LHCb Collaboration, R. Aaij et al., Phys. Rev. Lett. 115(11), 111803 (2015). arXiv:1506.08614 [Addendum: Phys. Rev. Lett. 115(15), 159901 (2015)]

110. D. Boubaa, S. Khalil, S. Moretti, arXiv: 1604.03416

111. F. Mahmoudi, Comput. Phys. Commun. 178, 745-754 (2008). arXiv:0710.2067

112. F. Mahmoudi, Comput. Phys. Commun. 180, 1718-1719 (2009) 
113. F. Mahmoudi, Comput. Phys. Commun.180, 1579-1613 (2009). arXiv:0808.3144. For an undated version see http://superiso. in2p3.fr/superiso3.4.pdf

114. Heavy Flavor Averaging Group (HFAG) Collaboration, Y. Amhis et al., arXiv: 1412.7515

115. P. Gambino, M. Misiak, Nucl. Phys. B 611, 338-366 (2001). arXiv:hep-ph/0104034

116. G. Degrassi, P. Gambino, G.F. Giudice, JHEP 12, 009 (2000). arXiv:hep-ph/0009337

117. LHCb, CMS Collaborations, V. Khachatryan et al., Nature 522, 68-72 (2015). arXiv:1411.4413

118. ATLAS Collaboration, M. Aaboud et al., Eur. Phys. J. C 76, 513 (2016). arXiv: 1604.04263

119. BaBar Collaboration, J.P. Lees et al., Phys. Rev. D 88(3), 031102 (2013). arXiv:1207.0698

120. Belle Collaboration, B. Kronenbitter et al., Phys. Rev. D 92(5), 051102 (2015). arXiv: 1503.05613

121. T. Blum, A. Denig, I. Logashenko, E. de Rafael, B. Lee Roberts, T. Teubner, G. Venanzoni, arXiv: 1311.2198

122. A.E. Dorokhov, A.E. Radzhabov, A.S. Zhevlakov, Sov. Phys. JETP Lett. 100, 133 (2014)

123. A.E. Dorokhov, A.E. Radzhabov, A.S. Zhevlakov, J. Phys. Conf. Ser. 678(1), 012054 (2016)

124. Muon g-2 Collaboration, G.W. Bennett et al., Phys. Rev. Lett. 92, 161802 (2004). arXiv:hep-ex/0401008

125. Muon g-2 Collaboration, G. Bennett et al. Phys. Rev. D 73, 072003 (2006). arXiv:hep-ex/0602035

126. M. Davier, A. Hoecker, B. Malaescu, Z. Zhang, Eur. Phys. J. C71, 1515 (2011). arXiv: 1010.4180

127. S.P. Martin, J.D. Wells, Phys. Rev. D 64, 035003 (2001). arXiv:hep-ph/0103067

128. G. Degrassi, G. Giudice, Phys. Rev. D 58, 053007 (1998). arXiv:hep-ph/9803384

129. S. Heinemeyer, D. Stöckinger, G. Weiglein, Nucl. Phys. B 690, 62-80 (2004). arXiv:hep-ph/0312264

130. S. Heinemeyer, D. Stöckinger, G. Weiglein, Nucl. Phys. B 699, 103-123 (2004). arXiv:hep-ph/0405255

131. D. Stöckinger, J. Phys. G 34, R45-R92 (2007). arXiv:hep-ph/ 0609168

132. O. Stål, G. Weiglein, L. Zeune, JHEP 09, 158 (2015). arXiv: 1506.07465

133. Tevatron Electroweak Working Group for the CDF and DØ Collaborations, arXiv: 1204.0042

134. ALEPH, DELPHI, L3, OPAL, SLD, LEP Electroweak Working Group, SLD Electroweak Group, SLD Heavy Flavour Group, S. Schael, et al.. Phys.Rept. 427, 257-454 (2006). arXiv:hep-ex/0509008. See http://lepewwg.web.cern.ch/ LEPEWWG/

135. S. Heinemeyer, W. Hollik, G. Weiglein, L. Zeune, JHEP 12, 084 (2013). arXiv: 1311.1663

136. M. Awramik, M. Czakon, A. Freitas, Phys. Rev. D 69, 053006 (2004). arXiv:hep-ph/0311148

137. J. Haestier, S. Heinemeyer, D. Stöckinger, G. Weiglein, JHEP 12, 027 (2005). arXiv:hep-ph/0508139

138. S. Heinemeyer, W. Hollik, D. Stöckinger, A. Weber, G. Weiglein, JHEP 08, 052 (2006). arXiv:hep-ph/0604147

139. P. Bechtle, O. Brein, S. Heinemeyer, G. Weiglein, K.E. Williams, Comput. Phys. Commun. 181, 138-167 (2010). arXiv:0811.4169

140. P. Bechtle, O. Brein, S. Heinemeyer, G. Weiglein, K.E. Williams, Comput. Phys. Commun. 182, 2605-2631 (2011). arXiv: 1102.1898

141. P. Bechtle, O. Brein, S. Heinemeyer, O. Stål, T. Stefaniak, G. Weiglein, K. Williams, PoSCHARGED2012, 024 (2012). arXiv: 1301.2345
142. P. Bechtle, O. Brein, S. Heinemeyer, O. Stål, T. Stefaniak, G. Weiglein, K.E. Williams, Eur. Phys. J. C 74(3), 2693 (2014). arXiv: 1311.0055

143. CMS Collaboration, V. Khachatryan et al., JHEP 10, 160 (2014). arXiv: 1408.3316

144. CMS Collaboration. CMS-PAS-HIG-14-029

145. Particle Data Group Collaboration, C. Patrignani et al., Chin Phys. C 40, 100001 (2016)

146. M. Drees, H. Dreiner, D. Schmeier, J. Tattersall, J.S. Kim, Comput. Phys. Commun. 187, 227-265 (2015). arXiv:1312.2591

147. A. Djouadi, M.M. Mühlleitner, M. Spira, Acta Phys. Polon. B38, 635-644 (2007). arXiv:hep-ph/0609292

148. M. Bahr et al., Eur. Phys. J. C 58, 639-707 (2008). arXiv:0803.0883

149. J. Bellm et al., arXiv: 1310.6877

150. P. Bechtle et al., Eur. Phys. J. C 76(2), 96 (2016)

151. S. Heinemeyer, W. Hollik, G. Weiglein, Phys. Lett. B 455, 179191 (1999). arXiv:hep-ph/9903404

152. S. Liebler, S. Profumo, T. Stefaniak, JHEP 04, 143 (2016). arXiv: 1512.09172

153. M. Misiak et al., Phys. Rev. Lett. 114(22), 221801 (2015). arXiv: 1503.01789

154. J.F. Donoghue, L.F. Li, Phys. Rev. D 19, 945 (1979)

155. M. Carena, S. Gori, N.R. Shah, C.E. Wagner, JHEP 03, 014 (2012). arXiv: 1112.3336

156. ATLAS Collaboration, G. Aad et al., JHEP 10, 130 (2013), arXiv:1308.1841 [Erratum: JHEP 01, 109(2014)]

157. ATLAS Collaboration, G. Aad et al., JHEP 09, 176 (2014). arXiv: 1405.7875

158. ATLAS Collaboration, G. Aad et al., JHEP 10, 189, (2013). arXiv: 1308.2631

159. ATLAS Collaboration, G. Aad et al., JHEP 09, 015 (2014). arXiv: 1406.1122

160. ATLAS Collaboration, G. Aad et al., JHEP 10, 024 (2014). arXiv: 1407.0600

161. CMS Collaboration, S. Chatrchyan et al., Eur. Phys. J. C 73(9), 2568 (2013). arXiv: 1303.2985

162. ATLAS Collaboration, G. Aad et al., JHEP 04, 169 (2014) arXiv: 1402.7029

163. ATLAS Collaboration, G. Aad et al., JHEP 06, 124 (2014). arXiv: 1403.4853

164. ATLAS Collaboration, G. Aad et al., JHEP 11, 118 (2014). arXiv: 1407.0583

165. ATLAS Collaboration, G. Aad et al., JHEP 05, 071 (2014). arXiv: 1403.5294

166. ATLAS Collaboration, G. Aad et al., JHEP 04, 116 (2015), arXiv: 1501.03555

167. CMS Collaboration, V. Khachatryan et al., JHEP 04, 124 (2015). arXiv: 1502.06031

168. ATLAS Collaboration, G. Aad et al., JHEP 10, 134 (2015). arXiv: 1508.06608

169. ATLAS Collaboration, M. Aaboud et al., Phys. Lett. B 759, 555574 (2016). arXiv: 1603.09203

170. ATLAS Collaboration, G. Aad et al., JHEP 03, 127 (2016) arXiv: 1512.03704

171. ATLAS Collaboration, G. Aad et al., Phys. Rev. Lett. 113(17), 171801 (2014). arXiv:1407.6583

172. E.L. Berger, T. Han, J. Jiang, Phys. Rev. D 71, 115012 (2005). arXiv:hep-ph/0312286

173. M. Flechl, R. Klees, M. Kramer, M. Spira, M. Ubiali, Phys. Rev. D 91(7), 075015 (2015). arXiv:1409.5615

174. S. Profumo, T. Stefaniak, Phys. Rev. D 94(9), 095020 (2016). arXiv: 1608.06945

175. A. Pilaftsis, Phys. Rev. D 93(7), 075012 (2016)

176. P.M. Ferreira, J.P. Silva, Eur. Phys. J. C 69, 45-52 (2010). arXiv: 1001.0574 
177. R. Barbieri, L.J. Hall, V.S. Rychkov, Phys. Rev. D 74, 015007 (2006). arXiv:hep-ph/0603188

178. ATLAS Collaboration, M. Aaboud et al., Eur. Phys. J. C 76(11), 585 (2016). arXiv: 1608.00890

179. ATLAS Collaboration, G. Aad et al., Phys. Rev. D 92(1), 012006 (2015). arXiv:1412.2641

180. ATLAS Collaboration, G. Aad et al., Phys. Rev. D 91(1), 012006 (2015). arXiv:1408.5191

181. ATLAS Collaboration, G. Aad et al., Phys. Rev. D 90(11), 112015 (2014). arXiv: 1408.7084

182. ATLAS Collaboration, G. Aad et al., JHEP 04, 117 (2015). arXiv:1501.04943

183. ATLAS Collaboration, G. Aad et al., JHEP 01, 069 (2015). arXiv: 1409.6212

184. ATLAS Collaboration, G. Aad et al., JHEP 08, 137 (2015). arXiv: 1506.06641

185. ATLAS Collaboration, G. Aad et al., Phys. Lett. B 749, 519-541 (2015). arXiv: 1506.05988

186. ATLAS Collaboration, G. Aad et al., Eur. Phys. J. C 75(7), 349 (2015). arXiv: 1503.05066
187. CDF Collaboration, T. Aaltonen et al., Phys. Rev. D 88(5), 052013 (2013). arXiv:1301.6668

188. C.M.S. Collaboration, S. Chatrchyan et al., JHEP 01, 096 (2014). arXiv: 1312.1129

189. CMS Collaboration, S. Chatrchyan et al., Phys. Rev. D 89(9), 092007 (2014). arXiv:1312.5353

190. CMS Collaboration, V. Khachatryan et al., Eur. Phys. J. C 74(10), 3076 (2014). arXiv: 1407.0558

191. CMS Collaboration, V. Khachatryan et al., Phys. Lett. B 744, 184 (2015). arXiv: 1410.6679

192. CMS Collaboration, S. Chatrchyan et al., JHEP 05, 104 (2014). arXiv: 1401.5041

193. CMS Collaboration, S. Chatrchyan et al., Phys. Rev. D 89, 012003 (2014). arXiv: 1310.3687

194. CMS Collaboration, V. Khachatryan et al., JHEP 09, 087 (2014). arXiv:1408.1682. [Erratum: JHEP 10, 106 (2014)]

195. DØ Collaboration, V. M. Abazov et al., Phys. Rev. D 88(5) 052011 (2013). arXiv:1303.0823 